Supporting Information

\title{
Asymmetric Umpolung Hydrogenation and Deuteration of Alkenes Catalyzed by Nickel
}

\author{
Siyu Guo, Xiuhua Wang and Jianrong Steve Zhou*
}

State Key Laboratory of Chemical Oncogenomics, Key Laboratory of Chemical Genomics, School of Chemical Biology and Biotechnology, Peking University Shenzhen Graduate School, Nanshan District, Shenzhen 518055, China. E-mail: jrzhou@pku.edu.cn

Division of Chemistry and Biological Chemistry, School of Physical and Mathematical Sciences Nanyang Technological University, 21 Nanyang Link, SPMS-CBC-06-03, Singapore 637371.

Experimental procedures

\section{Table of Contents}

I. General

II. Condition optimization for asymmetric umpolung hydrogenation of alkenes

III. Asymmetric umpolung hydrogenation of $\alpha, \beta$-unsaturated esters

IV. Asymmetric umpolung hydrogenation of $\alpha, \beta$-unsaturated ketones

V. Asymmetric umpolung hydrogenation of allylic alcohols

VI. Asymmetric umpolung deuteration of $\alpha, \beta$-unsaturated esters

XII. Reference 


\section{General}

All NMR spectra were acquired on Bruker AV $500 \mathrm{MHz}$, BBFO $400 \mathrm{MHz}$ NMR spectrometers. ${ }^{1} \mathrm{H}$ NMR (500 $\mathrm{MHz})$ chemical shifts were recorded relative to $\mathrm{SiMe}_{4}(\delta 0.00)$ or residual protiated solvents $\left(\mathrm{CDCl}_{3}: \delta 7.26\right)$. Multiplicities were given as: $\mathrm{s}$ (singlet), $\mathrm{d}$ (doublet), $\mathrm{t}$ (triplet), $\mathrm{q}$ (quartet) and $\mathrm{m}$ (multiplet). The number of protons (n) for a given resonance was indicated by $\mathrm{nH}$. Coupling constants were reported as a $J$ value in $\mathrm{Hz}$. ${ }^{13} \mathrm{C}$ NMR (125 MHz) chemical shifts were recorded relative to solvent resonance $\left(\mathrm{CDCl}_{3}: \delta 77.16\right)$.

Glassware was dried at $120{ }^{\circ} \mathrm{C}$ for at least $3 \mathrm{~h}$ before use. Dry THF was freshly distilled from sodium/benzophenone under argon before use and stored in an argon filled glove box. Anhydrous 1,4-dioxane (Aldrich) was stored over activated $4 \AA$ molecular sieve beads in an argon-filled glove box. Dry toluene was collected from a solvent purification system containing a column of activated alumina ( $1 \mathrm{~m} \mathrm{x} 2)$ under argon. Methanol, ethanol and isopropanol were degassed and stored over dried molecular sieve in an argon-filled glove box before use. All anhydrous solvents were stored in Schlenk tubes in the glove box.

Unless noted otherwise, commercially available chemicals were used as received without purification. The GC internal standard, $n-\mathrm{C}_{12} \mathrm{H}_{26}$ and $n-\mathrm{C}_{14} \mathrm{H}_{30}$ was degassed with argon and dried over activated $4 \AA$ molecular sieve beads before use. Flash chromatography was performed using Merck 40-63D $60 \AA$ silica gel. Gas chromatography (GC) analysis was performed on a Shimadzu GC-2010 instrument with Agilent J \& W GC column DB-5MS-UI. GC/MS analysis was conducted on a Thermo Scientific DSQ II single quadrupole GC/MS instrument with Agilent J \& W GC column DB-5MS-UI. ESI/MS analysis was conducted on a Thermo Finnigan LCQ Fleet MS spectrometer. High resolution mass spectral analysis (HRMS) was performed on Finnigan MAT 95 XP mass spectrometer (Thermo Electron Corporation). Chiral HPLC analysis was performed on a Shimadzu LC-20AD instrument using Daicel Chiracel columns at $25^{\circ} \mathrm{C}$ and a mixture of HPLC-grade hexanes and isopropanol as eluent. Optical rotation was measured using a JASCO P-1030 Polarimeter equipped with a sodium vapor lamp at $589 \mathrm{~nm}$ and the concentration of samples was denoted as $c$. 


\section{Condition optimization for asymmetric umpolung hydrogenation of alkenes}

Condition optimization of a model $\alpha, \beta$-unsaturated ester: In an argon-filled glove box, $\mathrm{NiBr}_{2}(\mathrm{DME})(0.9 \mathrm{mg}$, $0.003 \mathrm{mmol}),(R)-\mathrm{Me}-\mathrm{DuPhos}(0.9 \mathrm{mg}, 0.003 \mathrm{mmol})$ and dry THF $(0.3 \mathrm{~mL})$ were charged into a $10-\mathrm{mL}$ reaction tube. After stirring for $10 \mathrm{~min}$, tetra- $n$-butylammonium iodide ( $7.4 \mathrm{mg}, 0.02 \mathrm{mmol})$, indium powder (17 mg, $0.15 \mathrm{mmol}$, Alfa Aesar, $100 \mathrm{mesh}, 99.9 \%$ purity), (E)-ethyl 3-methylcinnamate (19 mg, $0.1 \mathrm{mmol}$ ), GC standard $n-\mathrm{C}_{12} \mathrm{H}_{26}(10 \mu \mathrm{L})$, water ( 3 equiv, $\left.0.3 \mathrm{mmol}\right)$ and acetic acid $(17 \mu \mathrm{L}, 0.3 \mathrm{mmol})$ were added. The reaction mixture was heated with stirring in an oil bath maintained at $80{ }^{\circ} \mathrm{C}$. Aliquots were taken from the reaction mixture after $12 \mathrm{~h}$ in the glove box and passed through a short plug of silica gel with $\mathrm{Et}_{2} \mathrm{O}$ washing (about $3 \mathrm{~mL}$ ). The filtrate was subjected to GC analysis to determine the conversion of the olefin and the yield of the product. To determine the enantioselectivity of product, the filtered sample was subjected to chiral HPLC analysis (Daicel Chiralcel OD-H; $2 \% i$-PrOH in hexanes; flow rate $0.5 \mathrm{~mL} / \mathrm{min}$ ). The racemic product was prepared by hydrogenation with hydrogen gas ( 8 bar) over $10 \% \mathrm{Pd} / \mathrm{C}$ in methanol at room temperature for $12 \mathrm{~h}$.

Table S1 The effect of chiral ligands in umpolung hydrogenation of a model $\alpha, \beta$-unsaturated ester standard conditions
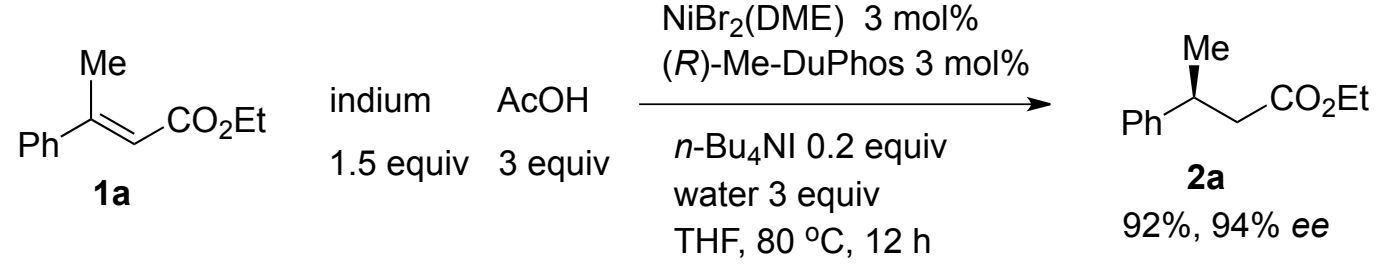

\begin{tabular}{|c|c|c|c|c|}
\hline Entry & Ligand & Conv (\%) & Yield (\%) & Ee (\%) \\
\hline 1 & & 92 & 92 & \\
\hline 2 & \\
\hline
\end{tabular}




\begin{tabular}{|c|c|c|c|c|}
\hline 3 & (R)-iPr-DuPhos & 0 & 0 & - \\
\hline 4 & & 72 & 72 & 86 \\
\hline 5 & $\begin{array}{l}\mathrm{H}_{3} \mathrm{C} \longrightarrow{ }_{(R)-\mathrm{Et}-\mathrm{BPE}} \\
\mathrm{CH}_{3}\end{array}$ & 43 & 42 & 86 \\
\hline 6 & (S)-Ph-BPE & $<5$ & $<5$ & - \\
\hline 7 & $\begin{array}{c}{ }_{\mathrm{C}}^{\stackrel{1}{3}} \\
(R) \text {-QuinoxP* }\end{array}$ & $<5$ & $<5$ & - \\
\hline 8 & $\begin{array}{c}t \text {-Bu } \quad \overline{\bar{t}} \text {-Bu } \\
(S) \text {-TangPhos }\end{array}$ & 0 & 0 & - \\
\hline 9 & (S)-DuanPhos & 0 & 0 & - \\
\hline 9 & $(R, S p)-\mathrm{CyPF}-\mathrm{Cy}$ & 0 & 0 & - \\
\hline
\end{tabular}




\begin{tabular}{|l|l|l|l|l|}
\hline 10 & 0 & 0 & \\
\hline 11 & $(R)$-BINAP & 0 & 0 \\
\hline
\end{tabular}

Table S2 The effect of nickel salts in umpolung hydrogenation of $\alpha, \beta$-unsaturated ester standard conditions

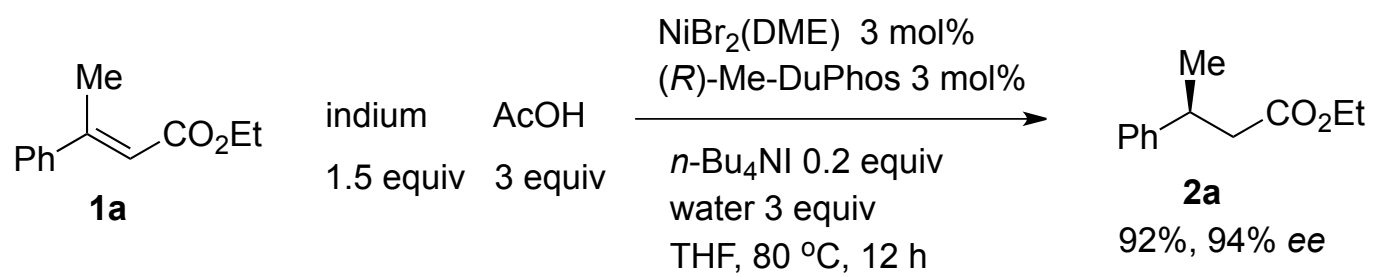

\begin{tabular}{|c|c|c|c|c|}
\hline Entry & Nickel salt & Conv (\%) & Yield (\%) & Ee (\%) \\
\hline 1 & $\mathrm{NiBr}_{2}(\mathrm{DME})$ & $\mathbf{9 2}$ & $\mathbf{9 2}$ & $\mathbf{9 4}$ \\
\hline 2 & $\mathrm{NiCl}_{2}(\mathrm{DME})$ & 78 & 77 & 94 \\
\hline 3 & $\mathrm{NiBr}_{2}$ & 63 & 62 & 94 \\
\hline 4 & $\mathrm{NiCl}_{2}$ & 57 & 57 & 92 \\
\hline 5 & $\mathrm{NiI}_{2}$ & 56 & 54 & 94 \\
\hline 6 & $\mathrm{Ni}(\mathrm{OTf})_{2}$ & 70 & 70 & 92 \\
\hline
\end{tabular}


Table S3 The effect of metal powder in umpolung hydrogenation of $\alpha, \beta$-unsaturated ester standard conditions

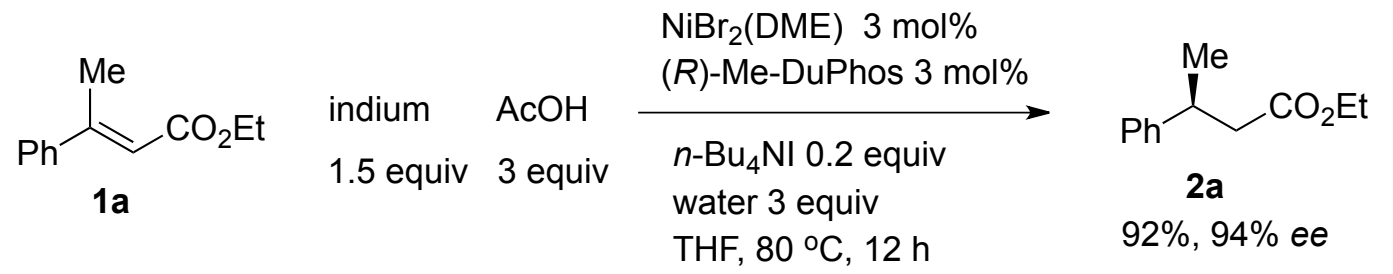

\begin{tabular}{|c|c|c|c|c|c|}
\hline Entry & Metal & Equivalent & Conv (\%) & Yield (\%) & Ee (\%) \\
\hline 1 & $\mathrm{Mg}$ & 0.5 & 13 & 12 & 47 \\
\hline 1 & $\mathrm{Mg}$ & 1.1 & 40 & $\mathbf{3 9}$ & $\mathbf{6 0}$ \\
\hline 2 & $\mathrm{Mn}$ & 0.5 & 27 & 26 & 80 \\
\hline 2 & $\mathrm{Mn}$ & 1.1 & 40 & $\mathbf{3 9}$ & $\mathbf{8 9}$ \\
\hline 3 & $\mathrm{Zn}$ & 0.5 & 13 & 13 & 87 \\
\hline 3 & $\mathrm{Zn}$ & 1.1 & 11 & 10 & 88 \\
\hline 4 & $\mathrm{In}$ & 0.5 & 52 & 52 & 94 \\
\hline 5 & $\mathrm{In}$ & 1.5 & $\mathbf{9 2}$ & $\mathbf{9 2}$ & $\mathbf{9 4}$ \\
\hline 6 & $\mathrm{In}$ & 3.0 & 86 & 86 & 94 \\
\hline
\end{tabular}


Table S4 The effect of acids in umpolung hydrogenation of $\alpha, \beta$-unsaturated ester standard conditions

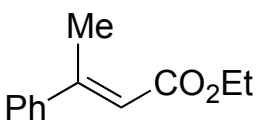
1a
$\mathrm{NiBr}_{2}$ (DME) $3 \mathrm{~mol} \%$
(R)-Me-DuPhos 3 mol\%
$\begin{array}{ll}\text { indium } & \mathrm{AcOH} \\ 1.5 \text { equiv } & 3 \text { equiv }\end{array}$
$n$ - $\mathrm{Bu}_{4} \mathrm{NI} 0.2$ equiv
water 3 equiv
THF, $80^{\circ} \mathrm{C}, 12 \mathrm{~h}$
$\overbrace{2 \mathrm{a}}^{\mathrm{Me}} \mathrm{CO}_{2} \mathrm{Et}$
$92 \%, 94 \%$ ee

\begin{tabular}{|c|c|c|c|c|}
\hline Entry & Acid & Conv (\%) & Yield (\%) & Ee (\%) \\
\hline 1 & AcOH & $\mathbf{9 2}$ & $\mathbf{9 2}$ & $\mathbf{9 4}$ \\
\hline 2 & $\mathrm{PivOH}$ & 78 & 77 & 92 \\
\hline 3 & $\mathrm{PhCO}_{2} \mathrm{H}$ & 65 & 65 & 93 \\
\hline 4 & $p-\mathrm{TsOH}$ & $<5$ & $<5$ & - \\
\hline 5 & $(\mathrm{COOH})_{2}$ & $<5$ & $<5$ & - \\
\hline 4 & Water & $<5$ & $<5$ & - \\
\hline
\end{tabular}

Table S5 The effect of $n$-Bu $\mathrm{u}_{4} \mathrm{NI}$ in umpolung hydrogenation of $\alpha, \beta$-unsaturated ester standard conditions

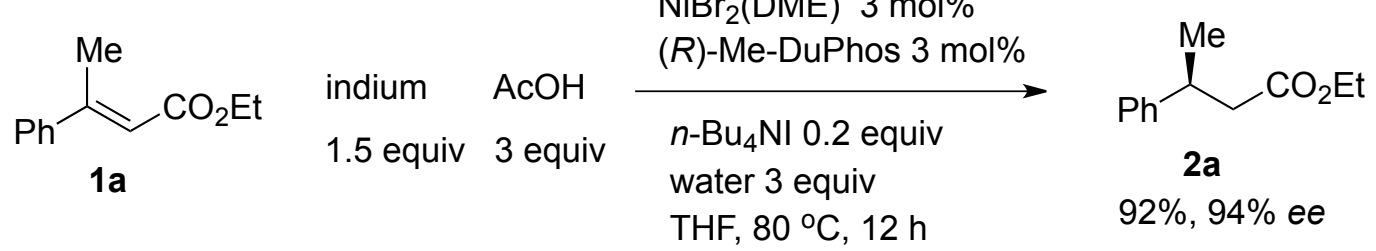

\begin{tabular}{|c|c|c|c|c|}
\hline Entry & $n$-Bu4NI (equiv) & Conv (\%) & Yield (\%) & Ee (\%) \\
\hline 1 & 0 & 29 & 29 & 93 \\
\hline 2 & 0.2 & $\mathbf{9 2}$ & $\mathbf{9 2}$ & $\mathbf{9 4}$ \\
\hline 3 & 0.5 & $\mathbf{9 2}$ & $\mathbf{9 2}$ & $\mathbf{9 4}$ \\
\hline
\end{tabular}


Table S6 The effect of chiral ligands in umpolung hydrogenation of 3-phenyl-2-butenol standard conditions
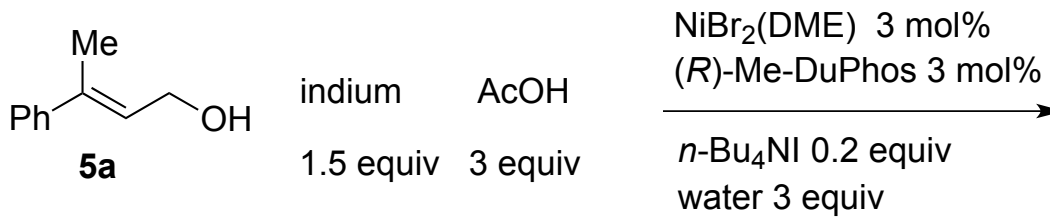<smiles>OCC[C@H]([AlH2])c1ccccc1</smiles>

6a $96 \%, 89 \%$ ee

$\mathrm{THF}, 60^{\circ} \mathrm{C}, 12 \mathrm{~h}$

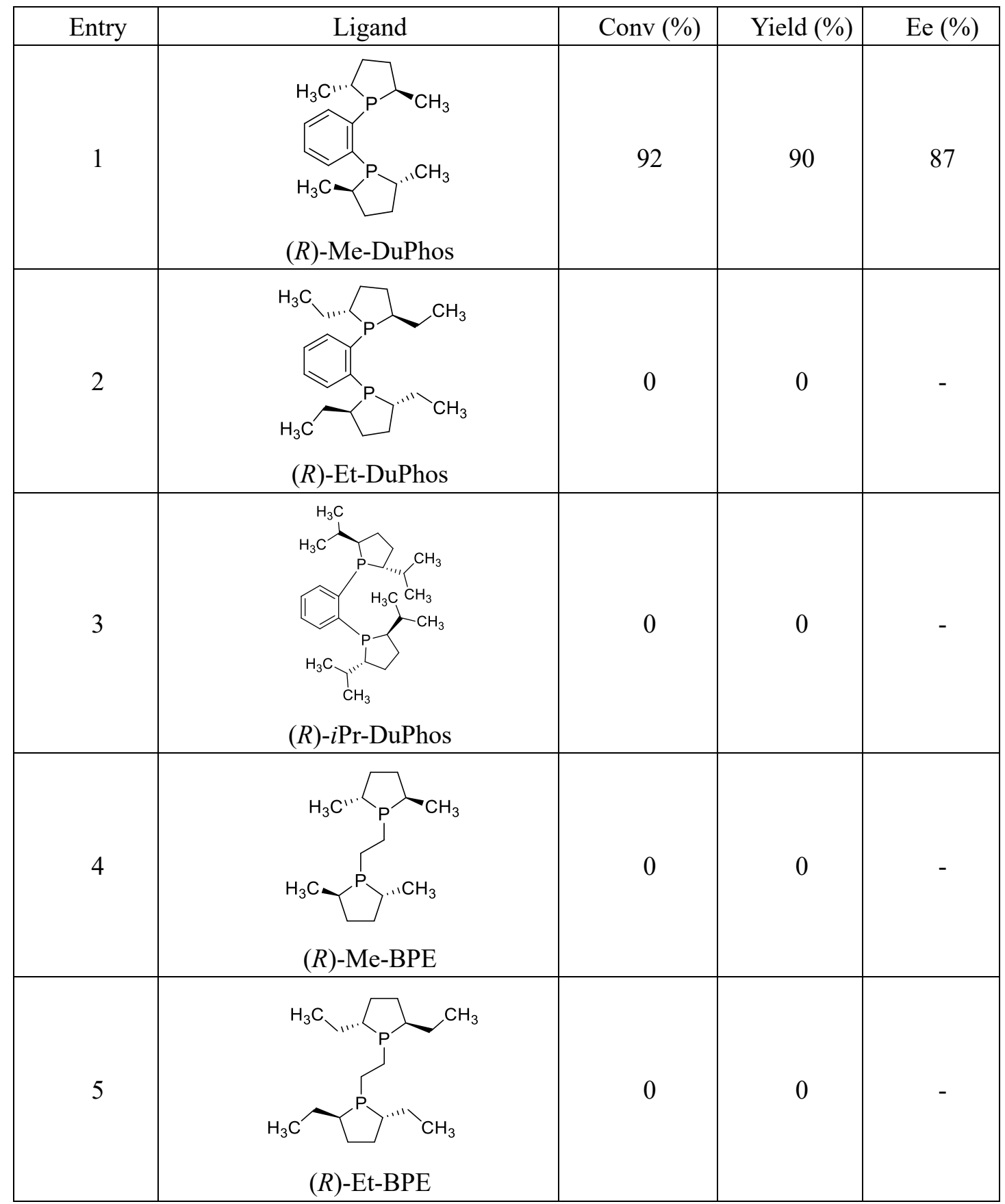




\begin{tabular}{|c|c|c|c|c|}
\hline 6 & 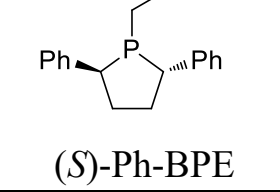 & 40 & 39 & 84 \\
\hline 7 & & 46 & 45 & 60 \\
\hline 8 & $\begin{array}{c}{ }_{t} \text {-Bu } \\
(S) \text {-TangPhos }\end{array}$ & 0 & 0 & - \\
\hline 9 & $(S)$-DuanPhos & 19 & 19 & 55 \\
\hline 9 & $(R, S p)-\mathrm{CyPF}-\mathrm{Cy}$ & 0 & 0 & - \\
\hline 10 & $(R)$-BINAP & 0 & 0 & - \\
\hline
\end{tabular}


Table S7 The effect of solvents in umpolung hydrogenation of 3-phenyl-2-butenol standard conditions<smiles>C/C(=C\CO)c1ccccc1</smiles>

$\mathrm{NiBr}_{2}$ (DME) $3 \mathrm{~mol} \%$ (R)-Me-DuPhos $3 \mathrm{~mol} \%$ $n-\mathrm{Bu}_{4} \mathrm{NI} 0.2$ equiv water 3 equiv $\mathrm{THF}, 60^{\circ} \mathrm{C}, 12 \mathrm{~h}$<smiles>CC(CCO)c1ccccc1</smiles>

6a $96 \%, 89 \%$ ee

\begin{tabular}{|c|c|c|c|c|}
\hline Entry & Solvent & Conv (\%) & Yield (\%) & Ee (\%) \\
\hline 1 & $\mathrm{MeOH}$ & 33 & 31 & 70 \\
\hline 2 & $\mathrm{EtOH}$ & 81 & 80 & 67 \\
\hline 3 & $i$-PrOH & 99 & 98 & 60 \\
\hline 4 & $\mathrm{CF}_{3} \mathrm{CH}_{2} \mathrm{OH}$ & 0 & 0 & - \\
\hline 5 & $\mathrm{PhCF}_{3}$ & 95 & 93 & 67 \\
\hline 6 & $\mathrm{MeCN}$ & 0 & 0 & - \\
\hline 7 & $\mathrm{DMF}$ & 47 & 46 & 84 \\
\hline 8 & $\mathrm{DMSO}$ & 3 & 3 & - \\
\hline 9 & $\mathrm{THF}$ & $\mathbf{9 2}$ & $\mathbf{9 0}$ & $\mathbf{8 9}$ \\
\hline 10 & Dioxane & 66 & 65 & 84 \\
\hline
\end{tabular}

Table S8 The effect of chiral ligands in umpolung hydrogenation of an enone standard conditions

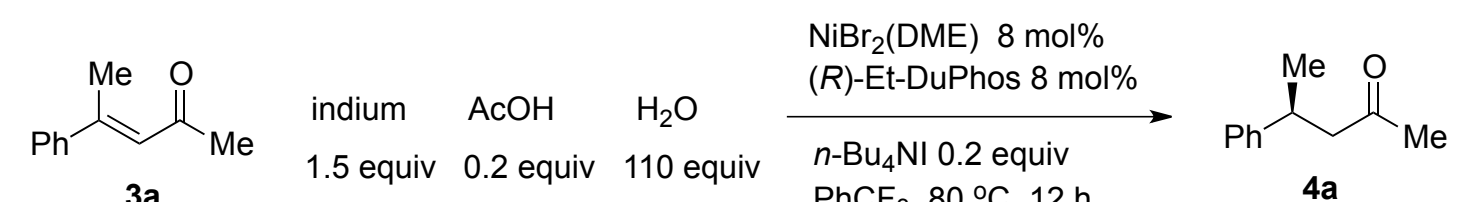

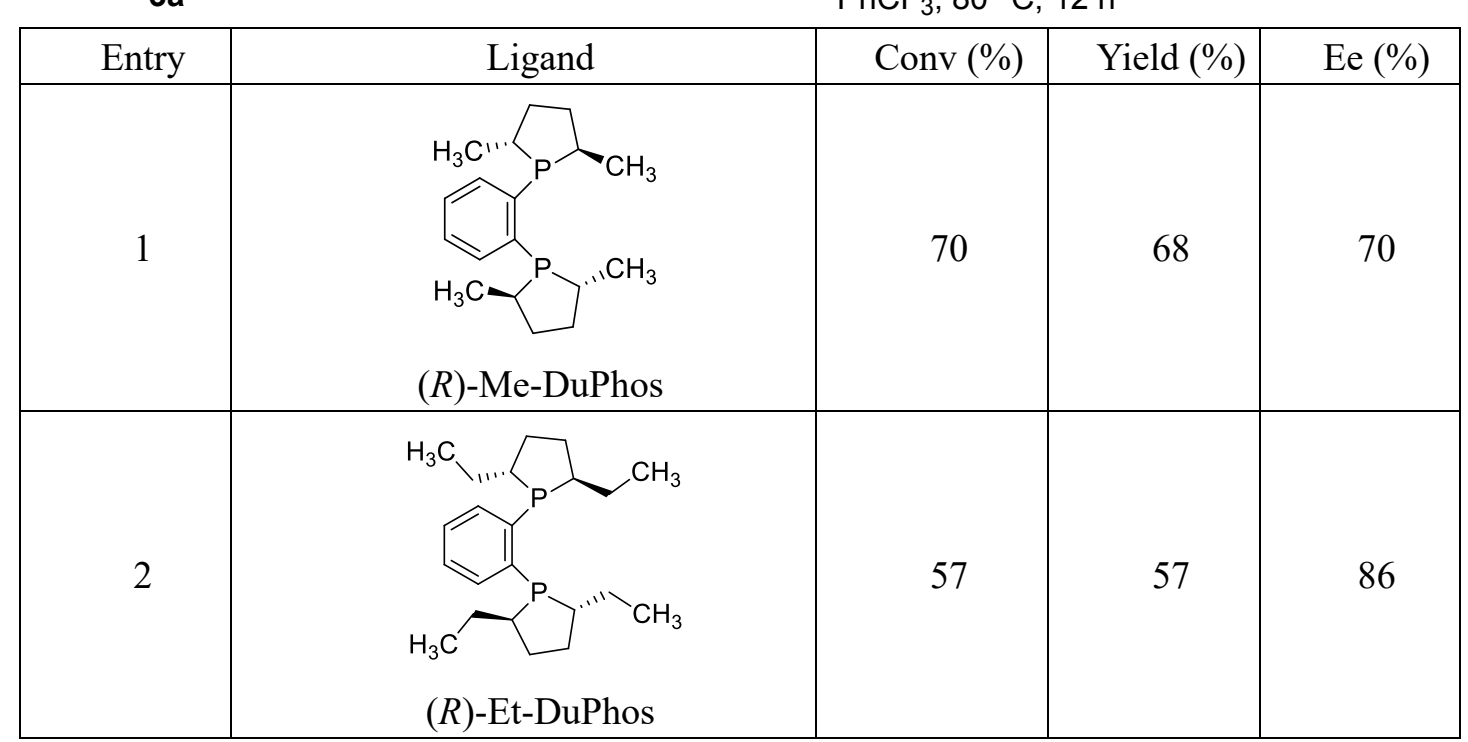




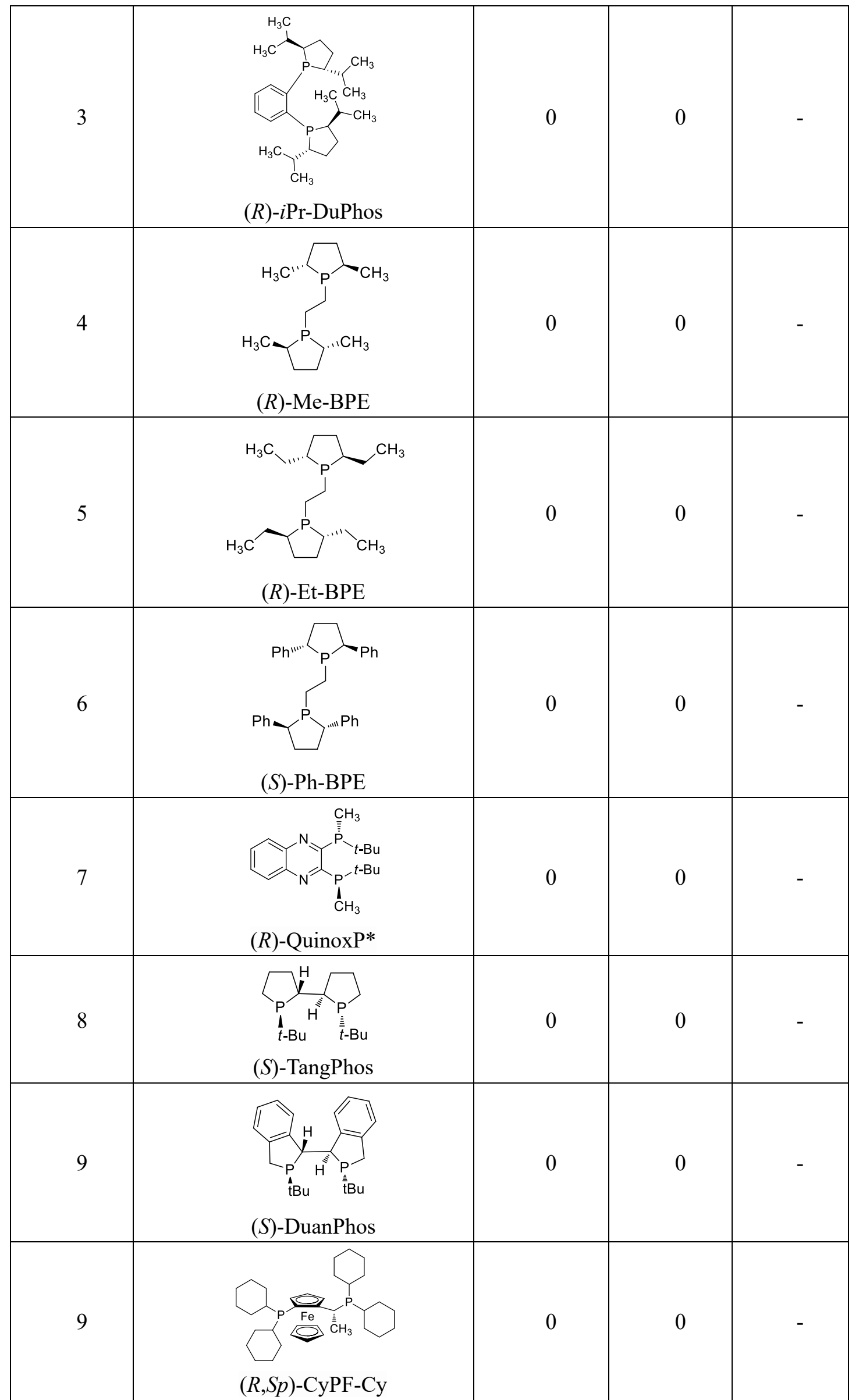




\begin{tabular}{|l|l|l|l|l|}
\hline 10 & $(R)$-BINAP & 0 & 0 \\
\hline
\end{tabular}

Table S9 The effect of solvents in umpolung hydrogenation of an enone standard conditions

\begin{tabular}{|c|c|c|c|c|}
\hline$\underbrace{\text { le }}_{3 a}$ & $\begin{array}{ll}\text { indium } & \mathrm{AcOH} \\
1.5 \text { equiv } & 0.2 \text { equi }\end{array}$ & $\begin{array}{l}\mathrm{H}_{2} \mathrm{O} \\
110 \text { equiv }\end{array}$ & $\begin{array}{l}\mathrm{NiBr}_{2}(\mathrm{DME}) 8 \mathrm{~m} \\
(R) \text {-Et-DuPhos } 8 \\
n-\mathrm{Bu}_{4} \mathrm{NI} 0.2 \mathrm{equ} \\
\mathrm{PhCF}_{3}, 80^{\circ} \mathrm{C}, 1\end{array}$ & \\
\hline Entry & Solvent & Conv $(\%)$ & Yield (\%) & Ee $(\%)$ \\
\hline 1 & $\mathrm{PhCF}_{3}$ & 58 & 57 & 84 \\
\hline 2 & THF & 11 & 9 & 83 \\
\hline 3 & 1,4-Dioxane & 0 & 0 & - \\
\hline 4 & $\mathrm{EtOH}$ & 0 & 0 & - \\
\hline 5 & $i$-PrOH & 36 & 34 & 84 \\
\hline 6 & DMF & 17 & 16 & 84 \\
\hline 7 & Ethyl acetate & 55 & 54 & 82 \\
\hline
\end{tabular}

Table S10 The effect of amounts of acetic acid in umpolung hydrogenation of an enone in $\mathrm{PhCF}_{3}$ standard conditions

\begin{tabular}{|c|c|c|c|c|}
\hline$\stackrel{\mathrm{le}}{\approx} \stackrel{11}{\mathrm{Me}}$ & $\begin{array}{ll}\text { indium } & \mathrm{AcO} \\
1.5 \text { equiv } & 0.2 \mathrm{e}\end{array}$ & $\begin{array}{l}\mathrm{H}_{2} \mathrm{O} \\
\text { v } 110 \text { equiv }\end{array}$ & $\begin{array}{l}\mathrm{NiBr}_{2}(\mathrm{DME}) 8 \mathrm{~m} \\
(R) \text {-Et-DuPhos } 8 \\
n-\mathrm{Bu}_{4} \mathrm{NI} 0.2 \text { equ } \\
\mathrm{PhCF}_{3}, 80^{\circ} \mathrm{C}, 1\end{array}$ & \\
\hline Entry & $\mathrm{AcOH}$ (equiv) & Conv $(\%)$ & Yield (\%) & $\mathrm{Ee}(\%)$ \\
\hline 1 & 0.2 & 54 & 54 & 84 \\
\hline 2 & 0.3 & 43 & 39 & 87 \\
\hline 3 & 0.5 & 27 & 27 & 86 \\
\hline 4 & 0.7 & 25 & 24 & 86 \\
\hline 5 & 1.0 & 25 & 25 & 84 \\
\hline
\end{tabular}


Table S11 Comparison with umpolung hydrogenation of an enone using formic acid

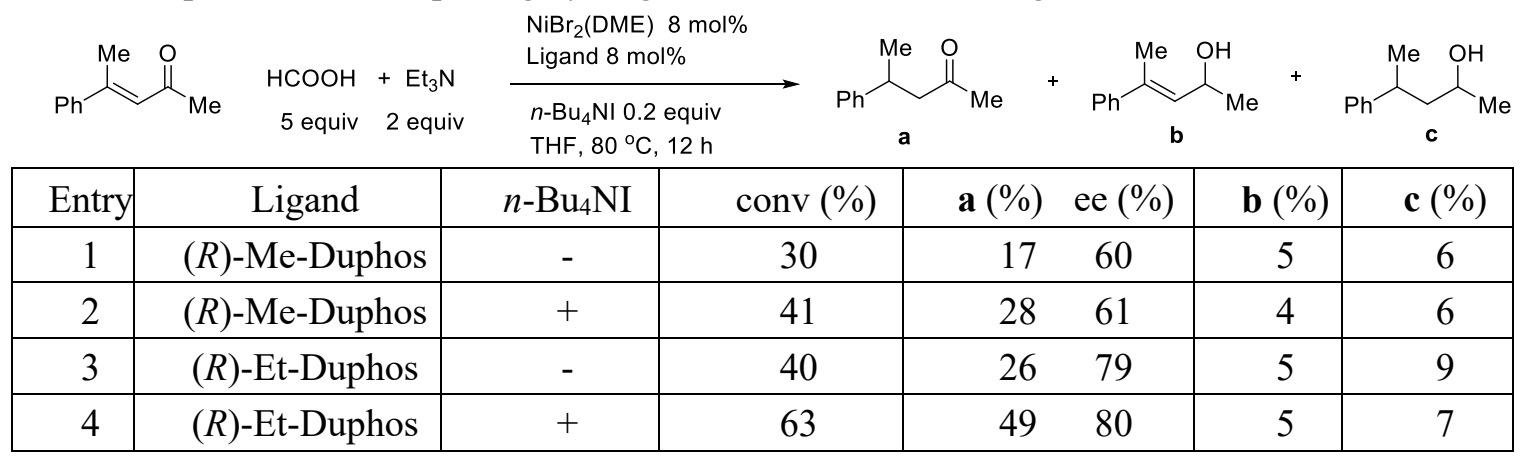

A procedure for nickel-catalyzed umpolung hydrogenation of enones using formic acid: In an argon-filled glove box, $\mathrm{NiBr}_{2}$ (DME) (2.4 mg, $\left.0.008 \mathrm{mmol}\right),(R)$-Me-DuPhos (2.4 mg, $\left.0.008 \mathrm{mmol}\right)$ or $(R)$-Et-DuPhos ( $2.9 \mathrm{mg}, 0.008 \mathrm{mmol})$, tetra- $n$-butylammonium iodide $(7.3 \mathrm{mg}, 0.02 \mathrm{mmol})$ and dry THF $(0.3 \mathrm{~mL})$ were charged into a reaction. After stirring for $15 \mathrm{~min}$ at room temperature, formic acid $(19 \mu \mathrm{L}, 0.5 \mathrm{mmol}, 5$ equiv), triethylamine ( $28 \mu \mathrm{L}, 0.2 \mathrm{mmol}, 2$ equiv) and $\alpha, \beta$-unsaturated ketone ( $0.1 \mathrm{mmol})$ were added. The reaction mixture was heated with vigorous stirring in an oil bath maintained at $80{ }^{\circ} \mathrm{C}$ for $12 \mathrm{~h}$. After the reaction mixture was cooled to room temperature, solid $\mathrm{NaHCO}_{3}$ was added to basify the mixture. The filtrate was subjected to GC analysis to determine the conversion of the olefin and the yield of the product. To determine the ee of product, the filtered sample was subjected to chiral HPLC analysis. 


\section{Asymmetric umpolung hydrogenation of $\alpha, \beta$-unsaturated esters}

A general procedure on $0.3 \mathrm{mmol}$ scale: In an argon-filled glove box, $\mathrm{NiBr}_{2}(\mathrm{DME})(2.8 \mathrm{mg}, 0.009 \mathrm{mmol}, 3$ mol\%), $(R)$-Me-DuPhos (2.8 mg, $0.009 \mathrm{mmol}, 3 \mathrm{~mol} \%)$ and dry THF $(0.6 \mathrm{~mL})$ were charged into a dry 10$\mathrm{mL}$ Schlenk tube. After stirring for $15 \mathrm{~min}$ at room temperature, tetra- $n$-butylammonium iodide $(22.2 \mathrm{mg}$, $0.06 \mathrm{mmol}$ ), indium powder (52 mg, $0.45 \mathrm{mmol}$, Alfa Aesar, $100 \mathrm{mesh}, 99.9 \%$ purity), Ethyl (E)-3phenylcrotonate $(0.3 \mathrm{mmol})$, water ( 3 equiv, $0.9 \mathrm{mmol})$ and acetic acid $(51 \mu \mathrm{L}, 0.9 \mathrm{mmol}, 3$ equiv) were added. The reaction mixture was heated with vigorous stirring in an oil bath maintained at $80{ }^{\circ} \mathrm{C}$ for $24 \mathrm{~h}$, until most or all of the olefin was consumed as monitored by GC. After the reaction mixture was cooled to room temperature, solid $\mathrm{NaHCO}_{3}$ was added to basify the mixture. After addition of silica gel and evaporation of the solvent on a rotary evaporator, the residue was dry loaded on a silica gel column and purified by flash chromatography using ethyl acetate and hexanes as eluent. The enantioselectivity of the purified product was determined by chiral HPLC analysis with Daicel Chiralcel columns. The use of Schlenk tubes and a vacuum manifold gave similar results with the glove box procedure. All the products herein have been isolated and fully characterized by our group previously. ${ }^{1} 92 \%$ yield and $94 \%$ ee.

A typical procedure on 1.0 mmol scale: In an argon-filled glove box, $\mathrm{NiBr}_{2}(\mathrm{DME})(3.1 \mathrm{mg}, 0.01 \mathrm{mmol}, 1$ mol\%), (R)-Me-DuPhos (3.1 mg, $0.01 \mathrm{mmol}, 1 \mathrm{~mol} \%)$ and ethanol $(1.0 \mathrm{~mL})$ were charged into a dry $10-\mathrm{mL}$ Schlenk tube. After stirring for $15 \mathrm{~min}$ at room temperature, tetra- $n$-butylammonium iodide (74 $\mathrm{mg}, 0.2$ mmol), indium powder (173 mg, $1.5 \mathrm{mmol}$, Alfa Aesar, 100 mesh, 99.9\% purity), ethyl (E)-3phenylcrotonate $(190 \mathrm{mg}, 1.0 \mathrm{mmol})$ and acetic acid $(172 \mu \mathrm{L}, 3 \mathrm{mmol}, 3$ equiv) were added. The reaction mixture was heated with vigorous stirring in an oil bath maintained at $80{ }^{\circ} \mathrm{C}$ for $24 \mathrm{~h}$, until $90 \%$ of the olefin was consumed as monitored by GC. After the reaction mixture was cooled to room temperature, solid $\mathrm{NaHCO}_{3}$ was added to basify the mixture. After addition of silica gel and evaporation of the solvent on a rotary evaporator, the residue was dry loaded on a silica gel column and purified by flash chromatography using ethyl acetate and hexanes as eluent to give $(S)$-Ethyl 3-phenylbutanoate 2a. $165 \mathrm{mg}, 86 \%$ yield and $92 \%$ ee. The enantioselectivity of the purified product was determined by chiral HPLC analysis with Daicel Chiralcel columns. The use of Schlenk tubes and a vacuum manifold gave similar results with the glove box procedure 
<smiles>CCOC(=O)C[C@H](C)c1ccccc1</smiles>

(S)-Ethyl 3-phenylbutanoate 2a [1134-71-0].

Ethyl (E)-3-phenylcrotonate $(58 \mathrm{mg}, 0.3 \mathrm{mmol})$ were used. The reaction was complete after $24 \mathrm{~h}$ at $80{ }^{\circ} \mathrm{C}$. The product was isolated by flash chromatography $\left(\mathrm{Et}_{2} \mathrm{O} /\right.$ hexanes $\left.1: 20\right)$ as colorless oil. $53 \mathrm{mg}, 92 \%$ yield $[\alpha]^{23} \mathrm{D}=+20.1^{\circ}\left(\mathrm{c}=0.86, \mathrm{CHCl}_{3}\right)$

94\% ee. Daicel Chiralcel OD-H, $n$-hexane/isopropanol 98/2, flow rate $=0.5 \mathrm{~mL} / \mathrm{min}$.

mAU

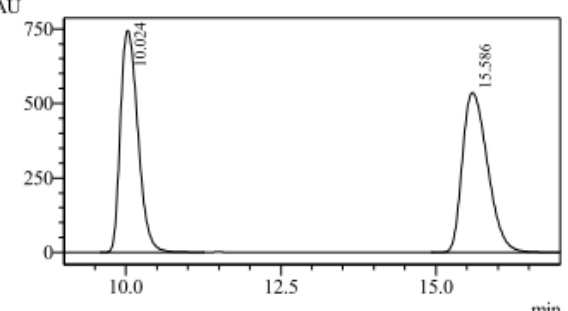

I. PDA Multi $1 / 200 \mathrm{~nm} 4 \mathrm{~nm}$

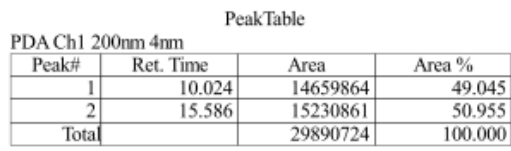

mAU

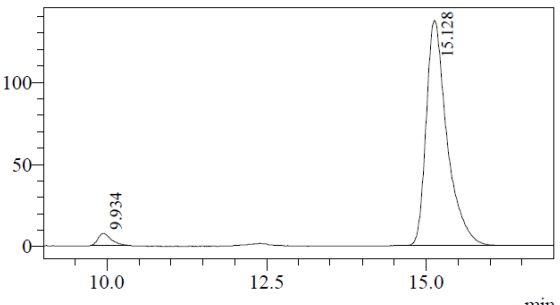

1 PDA Multi 1/200nm 4nm

PeakTable

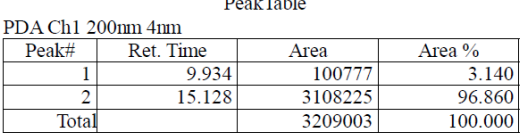

${ }^{1} \mathrm{H}$ NMR (400 MHz, $\left.\mathrm{CDCl}_{3}\right): \delta$ 7.34-7.25 (m, 2H), 7.25-7.15 (m, 2H), 4.08 (q, J=7.1 Hz, 2H), 3.34-3.21 (m, 1H), $2.61(\mathrm{dd}, J=15.0,7.0 \mathrm{~Hz}, 1 \mathrm{H}), 2.53(\mathrm{dd}, J=15.0,8.2 \mathrm{~Hz}, 1 \mathrm{H}), 1.30(\mathrm{~d}, J=7.0 \mathrm{~Hz}, 3 \mathrm{H}), 1.18(\mathrm{t}, J=7.1$ $\mathrm{Hz}, 3 \mathrm{H})$.

GC-MS (EI): Calcd for $\mathrm{C}_{12} \mathrm{H}_{16} \mathrm{O}_{2}\left[\mathrm{M}^{+}\right]$: 192.1 Found: 192.1.<smiles>COC(=O)C[C@H](C)c1ccccc1</smiles>

\section{(S)-Methyl 3-phenylbutanoate 2b [1441-20-9].}

Methyl (E)-3-phenylcrotonate $\left(53 \mathrm{mg}, 0.3 \mathrm{mmol}\right.$ ) were used. The reaction was complete after $24 \mathrm{~h}$ at $80{ }^{\circ} \mathrm{C}$. The product was isolated by flash chromatography $\left(\mathrm{Et}_{2} \mathrm{O} /\right.$ hexanes 1:20) as colorless oil. $49 \mathrm{mg}, 92 \%$ yield. $[\alpha]^{23}=+23.1^{\circ}\left(\mathrm{c}=0.68, \mathrm{CHCl}_{3}\right)$

Ee: 93\%. Daicel Chiralcel OD-H, $n$-hexane/isopropanol 98/2, flow rate $=0.5 \mathrm{~mL} / \mathrm{min}$. 
$\mathrm{mAL}$

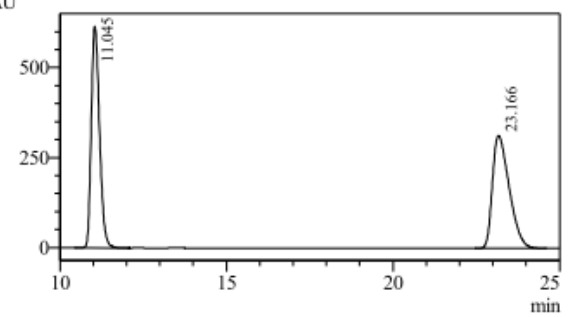

1 PDA Multi $1 / 200 \mathrm{~nm} 4 \mathrm{~nm}$

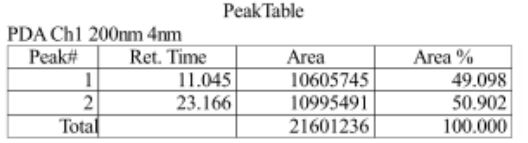

mAU

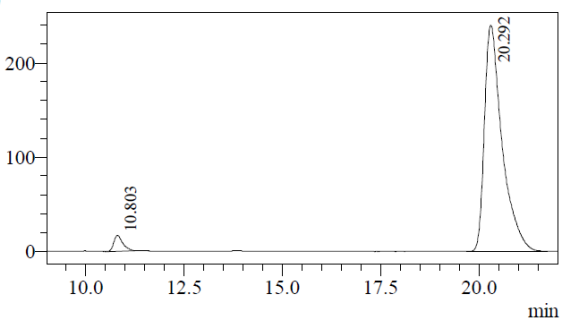

1 PDA Multi $1 / 200 \mathrm{~nm} 4 \mathrm{~nm}$

PeakTable

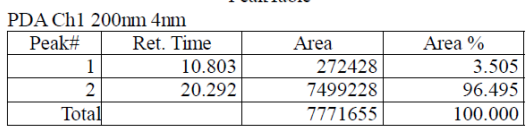

${ }^{1} \mathrm{H}$ NMR (400 MHz, $\left.\mathrm{CDCl}_{3}\right): \delta$ 7.36-7.27 (m, 2H), 7.24-7.16 (m, 3H), $3.62(\mathrm{~s}, 3 \mathrm{H}), 3.40-3.21(\mathrm{~m}, 1 \mathrm{H}), 2.63$ (dd, $J=15.2,6.9 \mathrm{~Hz}, 1 \mathrm{H}), 2.55$ (dd, $J=15.2,8.3 \mathrm{~Hz}, 1 \mathrm{H}), 1.30$ (d, $J=7.0 \mathrm{~Hz}, 3 \mathrm{H})$.

GC-MS (EI): Calcd for $\mathrm{C}_{11} \mathrm{H}_{14} \mathrm{O}_{2}[\mathrm{M}]^{+}:$178.1 Found: 178.1 .<smiles>[C+]C(CCOCC)c1ccccc1</smiles>

(S)-tert-Butyl 3-phenylbutanoate 2c [67217-41-8].

tert-Butyl (E)-3-phenylcrotonate $(65 \mathrm{mg}, 0.3 \mathrm{mmol})$ were used. The reaction was complete after $24 \mathrm{~h}$ at $80{ }^{\circ} \mathrm{C}$. The product was isolated by flash chromatography $\left(\mathrm{Et}_{2} \mathrm{O} /\right.$ hexanes 1:20) as colorless oil. $59 \mathrm{mg}, 90 \%$ yield $[\alpha]^{23}=+26.8^{\circ}\left(\mathrm{c}=0.85, \mathrm{CHCl}_{3}\right)$

Ee: $91 \%$. Daicel Chiralcel AS-H, $n$-hexane/isopropanol 90/10, flow rate $=0.5 \mathrm{~mL} / \mathrm{min}$.

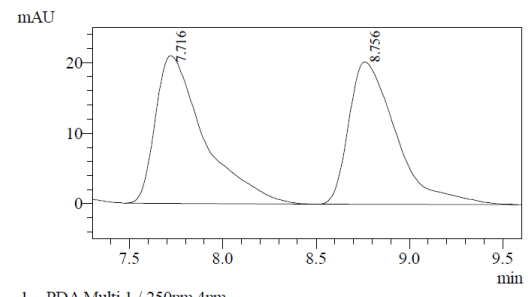

1 PDA Multi $1 / 250 \mathrm{~nm} 4 \mathrm{~nm}$

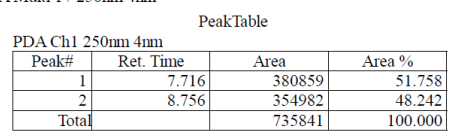

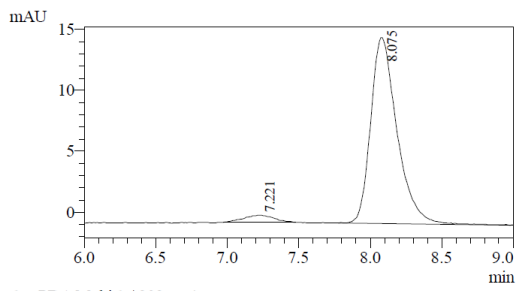

I PDA Multi 1 / 2091mu 4m

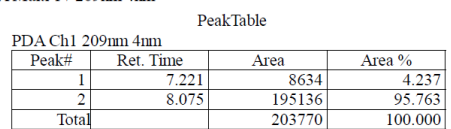

${ }^{1} \mathrm{H}$ NMR (400 MHz, $\left.\mathrm{CDCl}_{3}\right): \delta$ 7.35-7.29 (m, 2H), 7.28-7.19 (m, 3H), 3.34-3.17 (m, 1H), $2.56(\mathrm{dd}, J=14.7$, $7.3 \mathrm{~Hz}, 1 \mathrm{H}), 2.49(\mathrm{dd}, J=14.7,8.1 \mathrm{~Hz}, 1 \mathrm{H}), 1.39(\mathrm{~s}, 9 \mathrm{H}), 1.32$ (d, $J=7.0 \mathrm{~Hz}, 3 \mathrm{H})$.

GC-MS (EI): Calcd for $\mathrm{C}_{14} \mathrm{H}_{20} \mathrm{O}_{2}[\mathrm{M}]^{+}: 220.2$ Found: 220.1 . 
<smiles>CCOC(=O)C[C@H](C)c1ccc2ccccc2c1</smiles>

(S)-Ethyl 3-(2-naphthyl)butanoate 2d [1800576-17-3].

(E)-Ethyl 3-(2-naphthyl)-2-butenoate $(72 \mathrm{mg}, 0.3 \mathrm{mmol})$ were used. The reaction completed after $24 \mathrm{~h}$ at 80 ${ }^{\circ} \mathrm{C}$. The product was isolated by flash chromatography $\left(\mathrm{Et}_{2} \mathrm{O} /\right.$ hexanes $\left.1: 20\right)$ as colorless oil. $65 \mathrm{mg}$, $90 \%$ yield. $[\alpha]^{23}=+23.1^{\circ}\left(\mathrm{c}=0.58, \mathrm{CHCl}_{3}\right)$

Ee: $92 \%$. Daicel Chiralcel OJ-H, n-hexane/isopropanol 95/5, flow rate $=0.5 \mathrm{~mL} / \mathrm{min}$. mAL
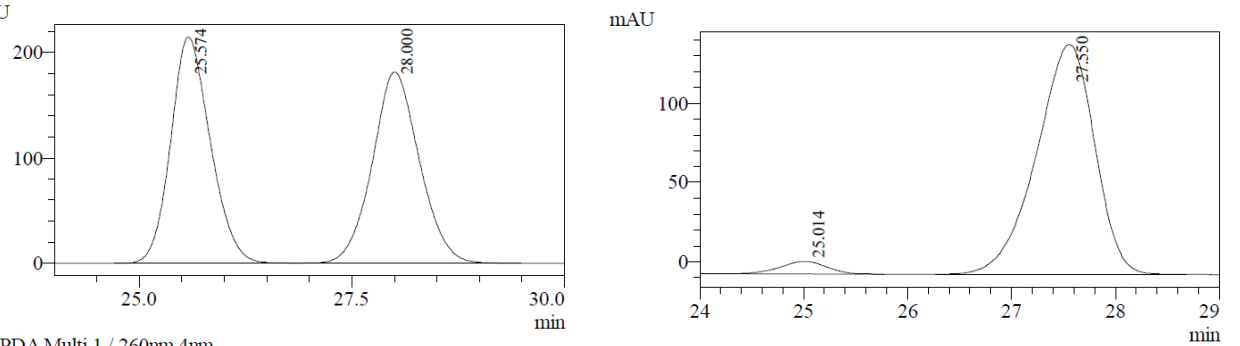

1 PDA Multi 1 / 260nm 4nm

PeakTable

1 PDA Multi $1 / 217 \mathrm{~nm} 4 \mathrm{~nm}$

PeakTable
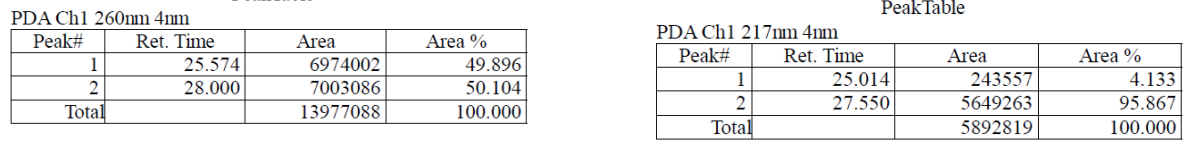

${ }^{1} \mathrm{H}$ NMR (400 MHz, $\left.\mathrm{CDCl}_{3}\right): \delta$ 7.91-7.72 (M, 3H), $7.65(\mathrm{~s}, 1 \mathrm{H})$, 7.54-7.29 (m, 3H), 4.17-3.95 (m, 2H), 3.56$3.32(\mathrm{~m}, 1 \mathrm{H}), 2.72(\mathrm{dd}, J=15.7,7.1 \mathrm{~Hz}, 1 \mathrm{H}), 2.65(\mathrm{dd}, J=15.08,8.1 \mathrm{~Hz}, 1 \mathrm{H}), 1.39(\mathrm{~d}, J=7.0 \mathrm{~Hz}, 3 \mathrm{H}), 1.17$ $(\mathrm{t}, J=7.1 \mathrm{~Hz}, 3 \mathrm{H})$.

GC-MS (EI): Calcd for $\mathrm{C}_{16} \mathrm{H}_{18} \mathrm{O}_{2}[\mathrm{M}]^{+}: 242.1$ Found: 242.1 .<smiles>CCOC(=O)C[C@H](C)c1ccc(C)cc1</smiles>

\section{(S)-Ethyl 3-(4-tolyl)butanoate 2e [1461-11-6].}

(E)-Ethyl 3-(4-tolyl)-2-butenoate $(61 \mathrm{mg}, 0.3 \mathrm{mmol})$ were used. The reaction completed after $24 \mathrm{~h}$ at $80{ }^{\circ} \mathrm{C}$. The product was isolated by flash chromatography $\left(\mathrm{Et}_{2} \mathrm{O} /\right.$ hexanes $\left.1: 20\right)$ as colorless oil. $56 \mathrm{mg}, 91 \%$ yield. $[\alpha]^{23}{ }_{\mathrm{D}}=+21.3^{\circ}\left(c=0.92, \mathrm{CHCl}_{3}\right)$

Ee: 91\%. Daicel Chiralcel OJ-H, n-hexane/isopropanol 99/1, flow rate $=0.5 \mathrm{~mL} / \mathrm{min}$. 


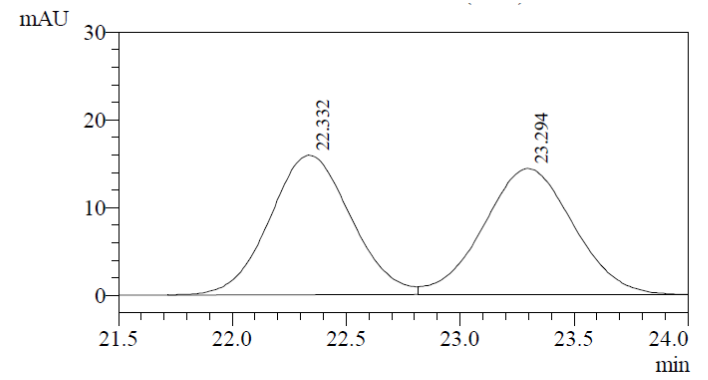

1 PDA Multi $1 / 250 \mathrm{~nm} 4 \mathrm{~nm}$

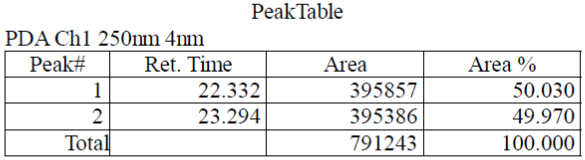

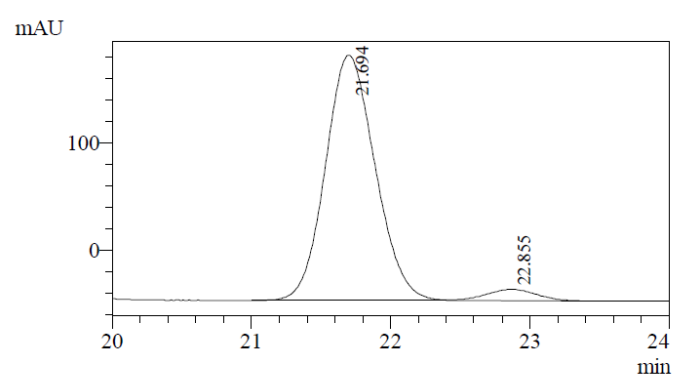

1 PDA Multi $1 / 200 \mathrm{~nm} 4 \mathrm{~nm}$

PeakTable

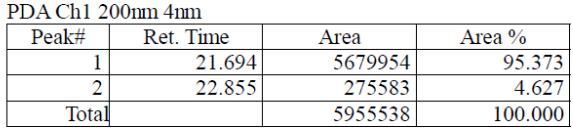

${ }^{1} \mathrm{H}$ NMR $\left(400 \mathrm{MHz}, \mathrm{CDCl}_{3}\right): \delta 7.11(\mathrm{~s}, 4 \mathrm{H}), 4.08(\mathrm{q}, J=7.1 \mathrm{~Hz}, 2 \mathrm{H}), 3.33-3.11(\mathrm{~m}, 1 \mathrm{H}), 2.49(\mathrm{dd}, J=15.0$, $7.0 \mathrm{~Hz}, 1 \mathrm{H}), 2.49(\mathrm{dd}, J=15.0,8.2 \mathrm{~Hz}, 1 \mathrm{H}), 2.31(\mathrm{~s}, 3 \mathrm{H}), 1.28(\mathrm{~d}, J=7.0 \mathrm{~Hz}, 3 \mathrm{H}), 1.19(\mathrm{~d}, J=7.1 \mathrm{~Hz}, 3 \mathrm{H})$. GC-MS (EI): Calcd for $\mathrm{C}_{13} \mathrm{H}_{18} \mathrm{O}_{2}[\mathrm{M}]^{+}: 206.1$ Found: 206.1 .<smiles>CCOC(=O)C[C@H](C)c1ccc(OC)cc1</smiles>

\section{(S)-Ethyl 3-(4-anisyl)butanoate 2f [1242409-36-4].}

(E)-Ethyl 3-(4-anisyl)-2-butenoate $(66 \mathrm{mg}, 0.3 \mathrm{mmol})$ were used. The reaction completed after $24 \mathrm{~h}$ at $80{ }^{\circ} \mathrm{C}$. The product was isolated by flash chromatography $\left(\mathrm{Et}_{2} \mathrm{O} /\right.$ hexanes $\left.1: 20\right)$ as colorless oil. $59 \mathrm{mg}, 88 \%$ yield $[\alpha]^{23}{ }_{\mathrm{D}}=+19.7^{\circ}\left(c=0.30, \mathrm{CHCl}_{3}\right)$

Ee: 92\%. Daicel Chiralcel OJ-H, $n$-hexane/isopropanol 99.5/0.5, flow rate $=0.5 \mathrm{~mL} / \mathrm{min}$.

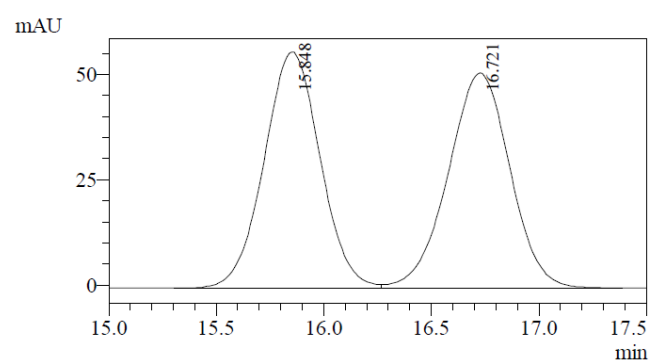

1 PDA Multi $1 / 216 \mathrm{~nm} 4 \mathrm{~nm}$

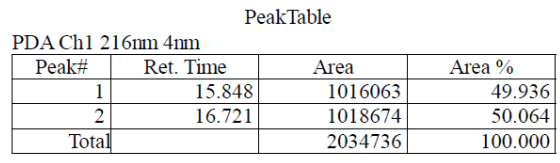

mAU

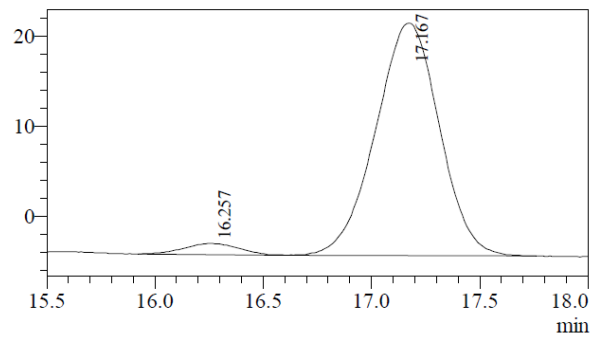

1 PDA Multi $1 / 218 \mathrm{~nm} 4 \mathrm{~nm}$

PeakTable

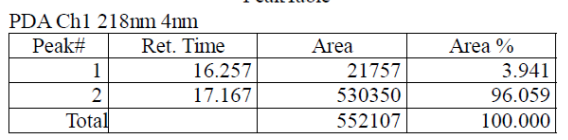

${ }^{1} \mathrm{H}$ NMR (400 MHz, $\left.\mathrm{CDCl}_{3}\right): \delta$ 7.18-7.09 (m, 2H), 6.91-6.79 (m, 2H), 4.12-4.01 (m, 2H), $3.78(\mathrm{~s}, 3 \mathrm{H}), 3.31-$ $3.16(\mathrm{~m}, 1 \mathrm{H}), 2.57(\mathrm{dd}, J=14.9,7.2 \mathrm{~Hz}, 1 \mathrm{H}), 2.50(\mathrm{dd}, J=14.9,8.0 \mathrm{~Hz}, 1 \mathrm{H}), 1.27$ (d, $J=7.0 \mathrm{~Hz}, 3 \mathrm{H}), 1.18(\mathrm{t}$, $J=7.1 \mathrm{~Hz}, 3 \mathrm{H})$. 
<smiles>CCOC(=O)C[C@H](C)c1ccc2c(c1)OCO2</smiles>

\section{(S)-Ethyl 3-(3,4-methylendioxyphenyl)butanoate $2 \mathrm{~g}$ [2013562-48-4].}

(E)-Ethyl 3-(3,4-methylendioxyphenyl)-2-butenoate (71 mg, $0.3 \mathrm{mmol})$ were used. The reaction completed after $24 \mathrm{~h}$ at $80^{\circ} \mathrm{C}$. The product was isolated by flash chromatography $\left(\mathrm{Et}_{2} \mathrm{O} /\right.$ hexanes 1:30) as colorless oil. $62 \mathrm{mg}, 87 \%$ yield

$[\alpha]^{23}=+26.6^{\circ}\left(\mathrm{c}=1.09, \mathrm{CHCl}_{3}\right)$

Ee: $92 \%$. Daicel Chiralcel OJ-H, $n$-hexane/isopropanol 90/10, flow rate $=1.0 \mathrm{~mL} / \mathrm{min}$.
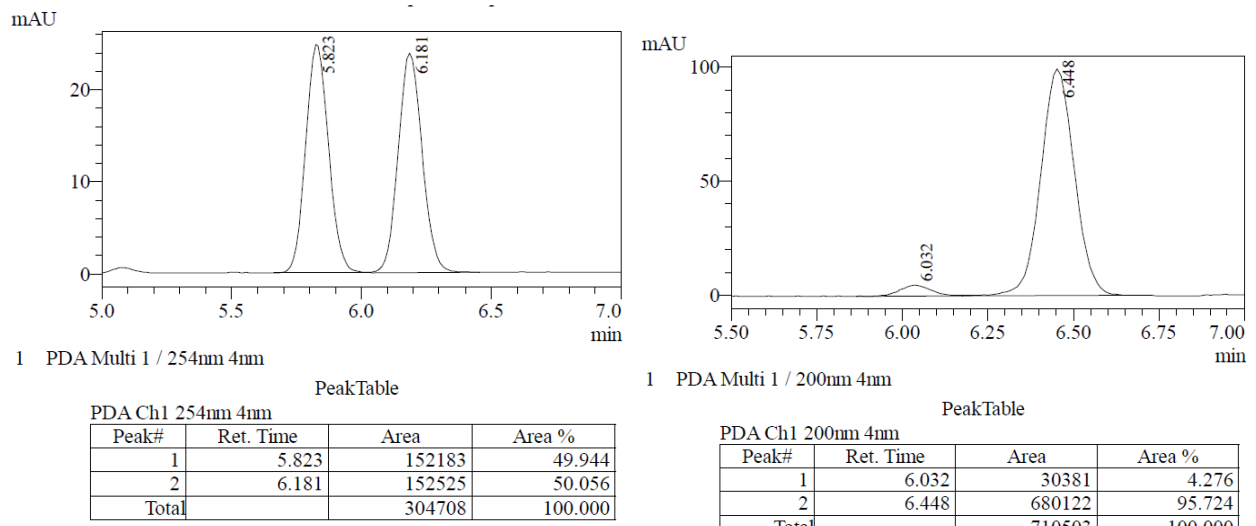

1 PDA Multi $1 / 200 \mathrm{~nm} 4 \mathrm{~nm}$

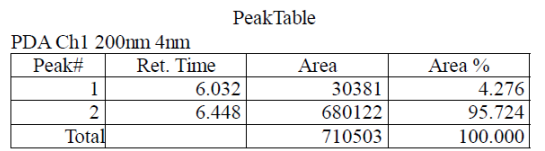

${ }^{1} \mathrm{H}$ NMR (400 MHz, $\left.\mathrm{CDCl}_{3}\right): \delta 6.71-6.61(\mathrm{~m}, 3 \mathrm{H}), 5.92(\mathrm{~s}, 2 \mathrm{H}), 4.08(\mathrm{q}, J=7.1 \mathrm{~Hz}, 2 \mathrm{H}), 3.28-3.13(\mathrm{~m}, 1 \mathrm{H})$, 2.60-2.43 (m, 2H), $1.26(\mathrm{~d}, J=7.0 \mathrm{~Hz}, 3 \mathrm{H}), 1.20(\mathrm{t}, J=7.1 \mathrm{~Hz}, 3 \mathrm{H})$.

GC-MS (EI): Calcd for $\mathrm{C}_{13} \mathrm{H}_{16} \mathrm{O}_{4}[\mathrm{M}]^{+}: 236.1$ Found: 236.0.<smiles>CCOC(=O)C[C@H](C)c1ccc(C(F)(F)F)cc1</smiles>

(S)-Ethyl 3-(4-trifluoromethylphenyl)butanoate $2 \mathrm{~h}$ [1800576-11-7].

(E)-Ethyl 3-(4-trifluoromethylphenyl)-2-butenoate $(77 \mathrm{mg}, 0.3 \mathrm{mmol})$ were used. The reaction completed after $24 \mathrm{~h}$ at $80^{\circ} \mathrm{C}$. The product was isolated by flash chromatography $\left(\mathrm{Et}_{2} \mathrm{O} /\right.$ hexanes 1:20) as colorless oil. $68 \mathrm{mg}$, $87 \%$ yield

$[\alpha]^{23}=+18.2^{\circ}\left(\mathrm{c}=0.49, \mathrm{CHCl}_{3}\right)$ 
Ee: 93\%. Daicel Chiralcel IC-H, n-hexane/isopropanol 99.9/0.1, flow rate $=0.5 \mathrm{~mL} / \mathrm{min}$.

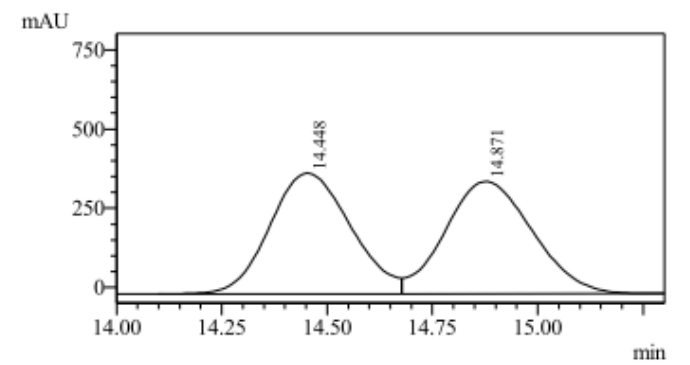

1 PDA Multi $1 / 200 \mathrm{~nm} 4 \mathrm{~nm}$

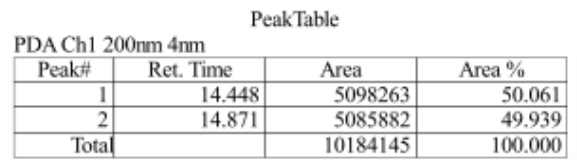

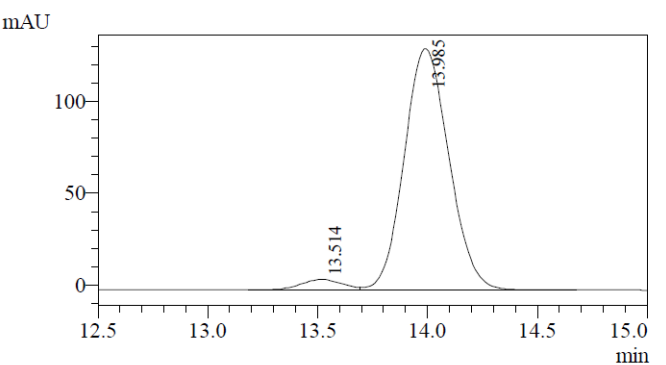

1 PDA Multi 1 / $210 \mathrm{~mm} 4 \mathrm{~mm}$

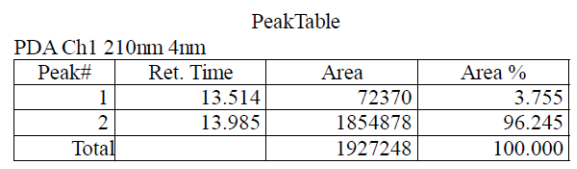

${ }^{1} \mathrm{H}$ NMR (400 MHz, $\left.\mathrm{CDCl}_{3}\right): \delta 7.55$ (d, $\left.J=8.1 \mathrm{~Hz}, 2 \mathrm{H}\right), 7.34(\mathrm{~d}, J=8.3 \mathrm{~Hz}, 2 \mathrm{H}), 4.07(\mathrm{q}, J=7.1 \mathrm{~Hz}, 2 \mathrm{H})$, 3.46-3.25 (m, 1H), $2.62(\mathrm{dd}, J=15.3,7.5 \mathrm{~Hz}, 1 \mathrm{H}), 2.56(\mathrm{dd}, J=15.3,7.7 \mathrm{~Hz}, 1 \mathrm{H}), 1.30(\mathrm{~d}, J=7.0 \mathrm{~Hz}, 3 \mathrm{H})$, $1.17(\mathrm{t}, J=7.1 \mathrm{~Hz}, 3 \mathrm{H})$.

GC-MS (EI): Calcd for $\mathrm{C}_{13} \mathrm{H}_{15} \mathrm{~F}_{3} \mathrm{O}_{2}[\mathrm{M}]^{+}: 260.1$ Found: 260.1 .<smiles>CCOC(=O)CC(C)c1cccc(C)c1</smiles>

(S)-Ethyl 3-(3-tolyl)butanoate 2i [130378-45-9].

(E)-Ethyl 3-( $m$-tolyl)but-2-enoate $(61 \mathrm{mg}, 0.3 \mathrm{mmol})$ were used. The reaction completed after $24 \mathrm{~h}$ at $80{ }^{\circ} \mathrm{C}$. The product was isolated by flash chromatography $\left(\mathrm{Et}_{2} \mathrm{O} /\right.$ hexanes $\left.1: 30\right)$ as colorless oil. $58 \mathrm{mg}, 93 \%$ yield $[\alpha]^{23}{ }_{\mathrm{D}}=+23.1^{\circ}\left(\mathrm{c}=0.58, \mathrm{CHCl}_{3}\right)$

Ee: 91\%. Daicel Chiralcel OD-H, $n$-hexane/isopropanol 90/10, flow rate $=0.5 \mathrm{~mL} / \mathrm{min}$.

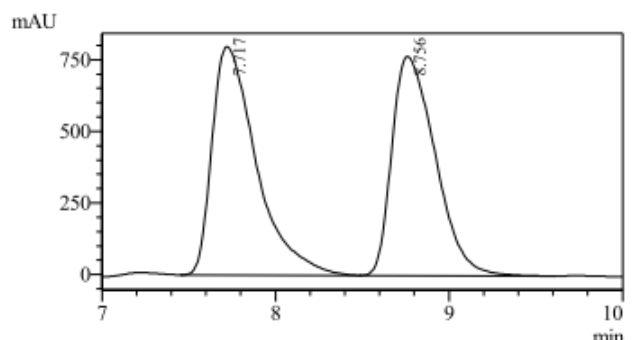

1 PDA Multi $1 / 200 \mathrm{~nm} 4 \mathrm{~nm}$

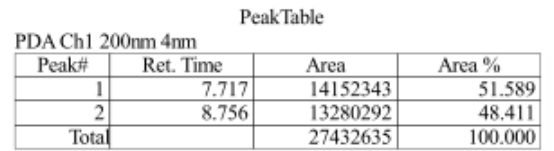
MAU

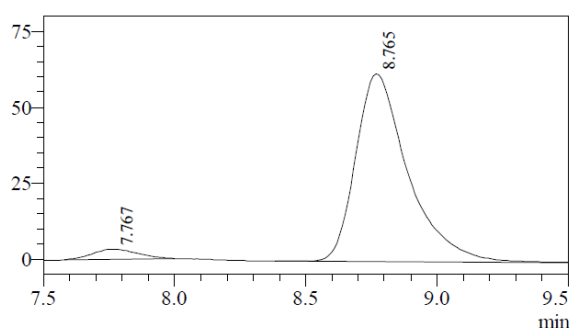

PDA Multi 1 / 210nm 4nm

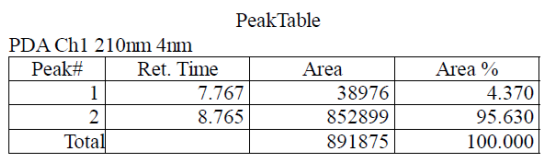

${ }^{1} \mathrm{H}$ NMR (400 MHz, $\left.\mathrm{CDCl}_{3}\right): \delta$ 7.23-7.13 (m, 1H), 7.05-6.90 (m, 3H), $4.08(\mathrm{q}, J=7.1 \mathrm{~Hz}, 2 \mathrm{H}), 3.33-3.13(\mathrm{~m}$, 
1H), $2.55(\mathrm{dd}, J=15.0,6.9 \mathrm{~Hz}, 1 \mathrm{H}), 2.48(\mathrm{dd}, J=15.0,8.3 \mathrm{~Hz}, 1 \mathrm{H}), 2.33(\mathrm{~s}, 3 \mathrm{H}), 1.29(\mathrm{~d}, J=7.0 \mathrm{~Hz}, 3 \mathrm{H})$, $1.19(\mathrm{~d}, J=7.1 \mathrm{~Hz}, 3 \mathrm{H})$.

GC-MS (EI): Calcd for $\mathrm{C}_{13} \mathrm{H}_{18} \mathrm{O}_{2}[\mathrm{M}]^{+}: 206.1$ Found: 206.1 .<smiles>CCOC(=O)C[C@H](C)c1cccs1</smiles>

\section{(S)-Ethyl 3-(2-thienyl)butanoate 2j [1800587-47-6].}

(E)-Ethyl 3-(2-thienyl)-2-butenoate $(59 \mathrm{mg}, 0.3 \mathrm{mmol})$ were used. The reaction completed after $24 \mathrm{~h}$ at $80{ }^{\circ} \mathrm{C}$. The product was isolated by flash chromatography $\left(\mathrm{Et}_{2} \mathrm{O} /\right.$ hexanes 1:20) as light yellow oil. $52 \mathrm{mg}, 88 \%$ yield $[\alpha]^{23}{ }_{\mathrm{D}}=+15.7^{\circ}\left(\mathrm{c}=0.36, \mathrm{CHCl}_{3}\right)$

Ee: 90\% ee. Daicel Chiralcel OD-H, $n$-hexane/isopropanol 99.5/0.5, flow rate $=0.5 \mathrm{~mL} / \mathrm{min}$.

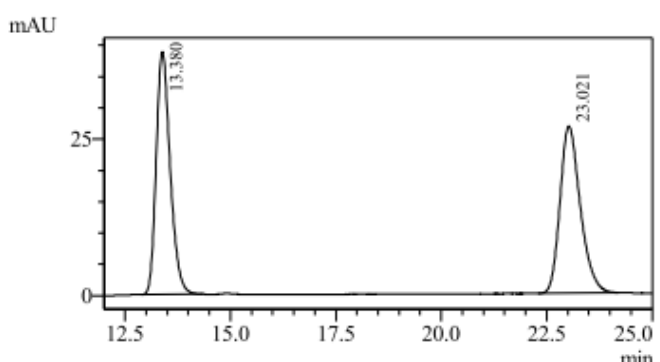

I PDA Multi $1 / 223 \mathrm{~nm} 4 \mathrm{~nm}$

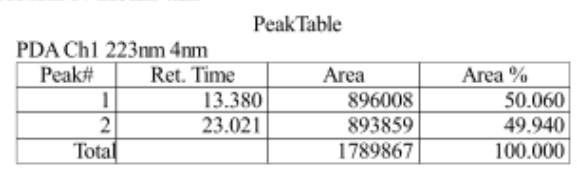

$\mathrm{mAU}$

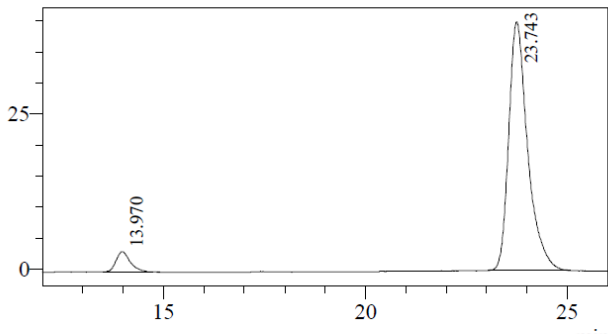

1 PDA Multi 1 / 226nm 4nm

PeakTable

PDACh $226 \mathrm{~nm} 4 \mathrm{~nm}$
\begin{tabular}{|r|r|r|r|}
\hline Peak\# & Ret. Time & \multicolumn{1}{|c|}{ Area } & Area $\%$ \\
\hline 1 & 13.970 & 78837 & 5.706 \\
\hline 2 & 23.743 & 1302873 & 94.294 \\
\hline Total & & 1381710 & 100.000 \\
\hline
\end{tabular}

${ }^{1} \mathrm{H}$ NMR (400 MHz, $\left.\mathrm{CDCl}_{3}\right): \delta$ 7.16-7.10 (m, 1H), 6.95-6.87 (m, 1H), 6.87-6.78 (m, 1H), 4.12 (q, J=7.1 Hz, 2H), 3.69-3.05 (m, 1H), $2.65(\mathrm{dd}, J=15.2,6.8 \mathrm{~Hz}, 1 \mathrm{H}), 2.55(\mathrm{dd}, J=15.2,8.0 \mathrm{~Hz}, 1 \mathrm{H}), 1.38(\mathrm{~d}, J=6.9 \mathrm{~Hz}$, $3 \mathrm{H}), 1.22(\mathrm{t}, J=7.1 \mathrm{~Hz}, 3 \mathrm{H})$.

GC-MS (EI): Calcd for $\mathrm{C}_{10} \mathrm{H}_{14} \mathrm{O}_{2} \mathrm{~S}[\mathrm{M}]^{+}$: 198.1 Found: 198.0.<smiles>CCOC(=O)C[C@H](C)c1cccnc1</smiles>

(S)-Ethyl 3-(3-pyridyl)butanoate 2k [1800576-18-4].

(E)-Ethyl 3-(3-pyridyl)-2-butenoate $(58 \mathrm{mg}, 0.3 \mathrm{mmol})$ were used. The reaction completed after $24 \mathrm{~h}$ at $80{ }^{\circ} \mathrm{C}$.

The product was isolated by flash chromatography $\left(\mathrm{Et}_{2} \mathrm{O} /\right.$ hexanes $\left.1: 5\right)$ as light yellow oil. $52 \mathrm{mg}, 90 \%$ yield. $[\alpha]^{23} \mathrm{D}=+23.2^{\circ}\left(\mathrm{c}=0.38, \mathrm{CHCl}_{3}\right)$ 
Ee: $92 \%$. Daicel Chiralcel AS-H, $n$-hexane/isopropanol 95/2, flow rate $=0.5 \mathrm{~mL} / \mathrm{min}$.

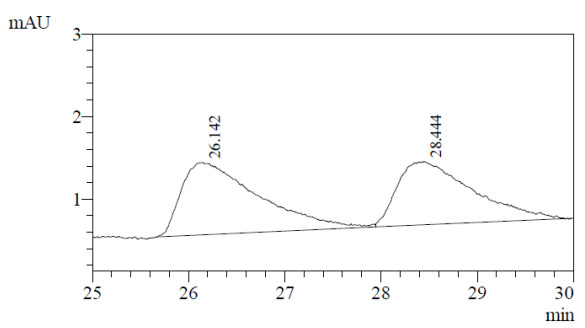

1 PDA Multi $1 / 210 \mathrm{~nm} 4 \mathrm{~nm}$

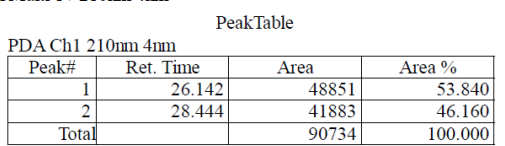

mAU

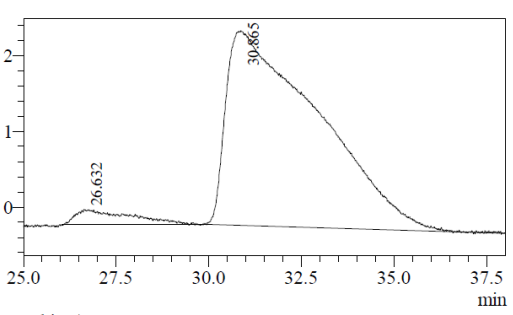

Multi $1 / 260 \mathrm{~mm} 4 \mathrm{~mm}$

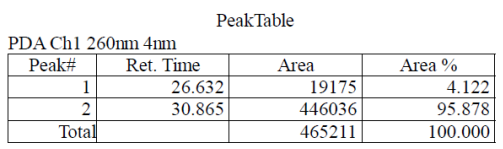

${ }^{1} \mathrm{H}$ NMR (400 MHz, $\left.\mathrm{CDCl}_{3}\right): \delta 8.52(\mathrm{~s}, 2 \mathrm{H}), 7.28-7.13(\mathrm{~m}, 2 \mathrm{H}), 4.08(\mathrm{q}, J=7.1 \mathrm{~Hz}, 2 \mathrm{H}), 3.35-3.16(\mathrm{~m}, 1 \mathrm{H})$, $2.61(\mathrm{dd}, J=15.5,7.4 \mathrm{~Hz}, 1 \mathrm{H}), 2.55(\mathrm{dd}, J=15.4,7.7 \mathrm{~Hz}, 1 \mathrm{H}), 1.30(\mathrm{~d}, J=7.0 \mathrm{~Hz}, 3 \mathrm{H}), 1.18$ (t, $J=7.1 \mathrm{~Hz}$, $3 \mathrm{H})$.

GC-MS (EI): Calcd for $\mathrm{C}_{11} \mathrm{H}_{15} \mathrm{NO}_{2}[\mathrm{M}]^{+}$: 193.1 Found: 193.1.<smiles>CCOC(=O)C[C@H]1CCCc2ccccc21</smiles>

(S)-Ethyl 2-(1-tetralinyl)acetate 21 [1800587-45-4].

The $(E)$-isomer of starting material was used $(65 \mathrm{mg}, 0.3 \mathrm{mmol})$. The product was isolated by flash chromatography $\left(\mathrm{Et}_{2} \mathrm{O} /\right.$ hexanes $\left.1: 15\right)$ as colorless oil. $58 \mathrm{mg}, 89 \%$ yield.

$[\alpha]^{23}=-0.22^{\circ}\left(\mathrm{c}=0.86, \mathrm{CHCl}_{3}\right)$

Ee: $85 \%$. Daicel Chiralcel OJ-H, $n$-hexane/isopropanol 98/2, flow rate $=1.0 \mathrm{~mL} / \mathrm{min}$.

mAt

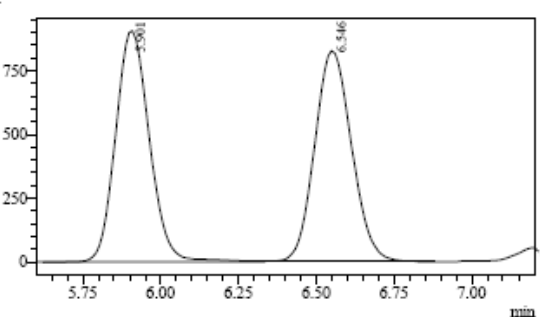

1 PDAMuriti $1 / 195 \mathrm{~mm} 4 \mathrm{~m}$

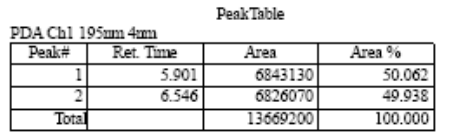

mAU

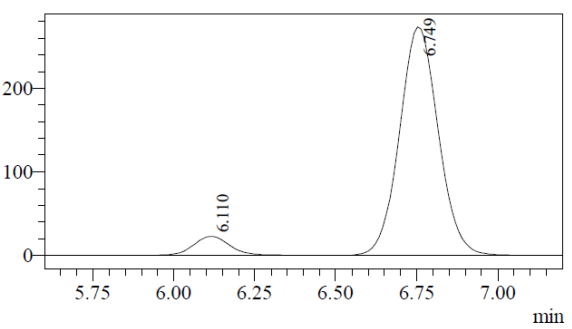

1 PDAMulti $1 / 200 \mathrm{~nm} 4 \mathrm{~nm}$

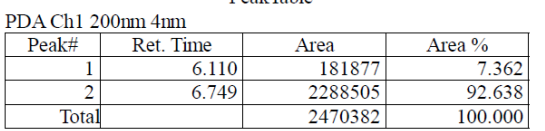


${ }^{1} \mathrm{H}$ NMR (400 MHz, $\left.\mathrm{CDCl}_{3}\right): \delta$ 7.21-7.00 (m, 4H), $4.17(\mathrm{q}, J=7.1 \mathrm{~Hz}, 2 \mathrm{H}), 3.42-3.24(\mathrm{~m}, 1 \mathrm{H}), 2.92-2.66(\mathrm{~m}$, $3 \mathrm{H}), 2.62-2.44(\mathrm{~m}, 3 \mathrm{H}), 2.54(\mathrm{dd}, J=15.2,9.9 \mathrm{~Hz}, 1 \mathrm{H}), 1.27(\mathrm{t}, J=7.1 \mathrm{~Hz}, 3 \mathrm{H})$.

GC-MS (EI): Calcd for $\mathrm{C}_{14} \mathrm{H}_{18} \mathrm{O}_{2}[\mathrm{M}]^{+}: 218.1$ Found: 218.0 .<smiles>CCOC(=O)C[C@H]1CCOc2ccccc21</smiles>

\section{(S)-Ethyl 2-(4-chromanyl)acetate 2m [1800576-12-8].}

The $(E)$-isomer of starting material was used. The product was isolated by flash chromatography (EtOAc/hexanes 1:4) as colorless oil. $63 \mathrm{mg}, 95 \%$ yield.

Ee: $84 \%$. Daicel Chiralcel OJ-H, $n$-hexane/isopropanol 90/10, flow rate $=1.0 \mathrm{~mL} / \mathrm{min}$.

$[\alpha]^{23}=+3.3^{\circ}\left(\mathrm{c}=0.58, \mathrm{CHCl}_{3}\right)$
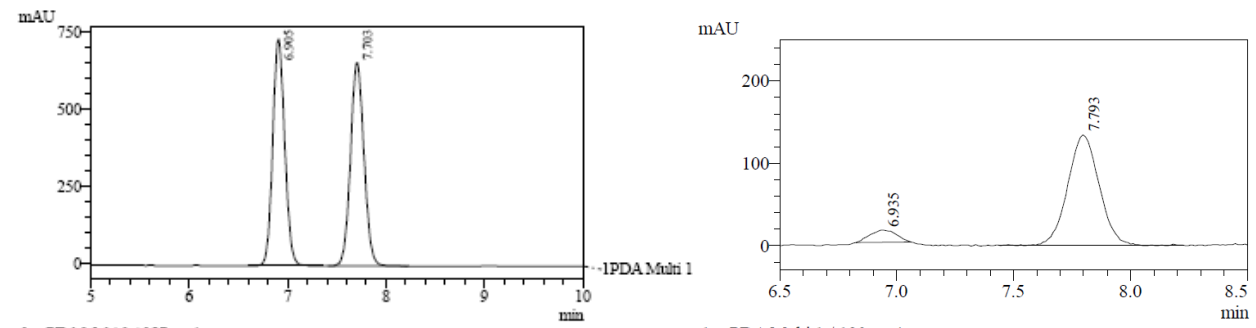

1 PDAMutit $1 / 197 \mathrm{~mm} 4 \mathrm{~mm}$
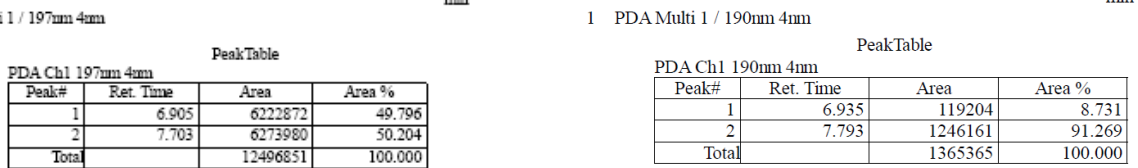

${ }^{1} \mathrm{H}$ NMR (400 MHz, $\left.\mathrm{CDCl}_{3}\right): \delta$ 7.16-7.04 (m, 2H), 6.92-6.75 (m, 2H), 4.25-4.11 (m, 4H), 3.43-3.29 (m, 1H), $2.79(\mathrm{dd}, J=15.6,4.7 \mathrm{~Hz}, 1 \mathrm{H}), 2.51,2.15(\mathrm{dd}, J=15.6,10.1 \mathrm{~Hz}, 1 \mathrm{H}), 2.32-2.05(\mathrm{~m}, 1 \mathrm{H}=\mathrm{H}), 1.98-1.76(\mathrm{~m}$, $1 \mathrm{H}), 1.27(\mathrm{t}, J=7.2 \mathrm{~Hz}, 3 \mathrm{H})$.

GC-MS (EI): Calcd for $\mathrm{C}_{13} \mathrm{H}_{16} \mathrm{O}_{3}[\mathrm{M}]^{+}: 220.1$ Found: 220.0.<smiles>CCOC(=O)CC(CC)c1ccccc1</smiles>

(S)-Ethyl 3-phenylpentanoate 2n [2845-23-0].

(E)-Ethyl 3-phenyl-2-pentenoate $(62 \mathrm{mg}, 0.3 \mathrm{mmol})$ were used. The reaction completed after $24 \mathrm{~h}$ at $80{ }^{\circ} \mathrm{C}$.

The product was isolated by flash chromatography $\left(\mathrm{Et}_{2} \mathrm{O} /\right.$ hexanes $\left.1: 20\right)$ as colorless oil. $54 \mathrm{mg}, 87 \%$ yield. $[\alpha]^{23}=14.5^{\circ}\left(\mathrm{c}=1.04, \mathrm{CHCl}_{3}\right)$ 
Ee: 93\%. Daicel Chiralcel OD-H, $n$-hexane/isopropanol 99/1, flow rate $=0.5 \mathrm{~mL} / \mathrm{min}$.

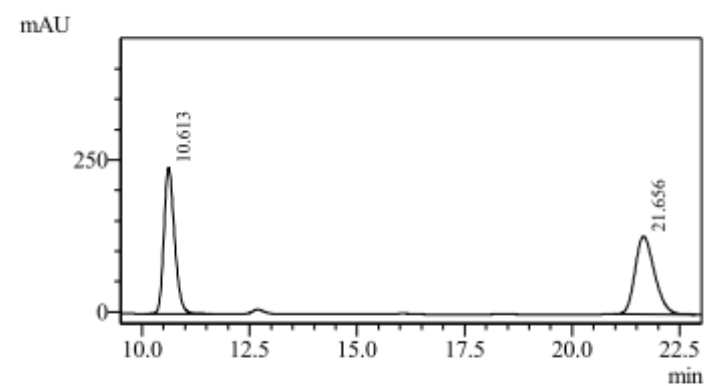

I PDA Multi $1 / 200 \mathrm{~nm} 4 \mathrm{~nm}$

PeakTable

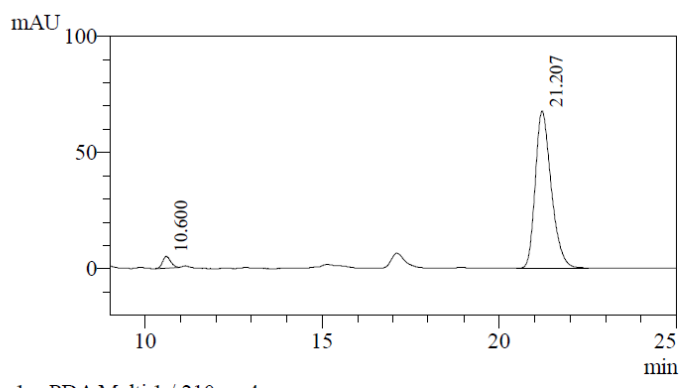

1 PDA Multi 1 / 210nm 4nm

PeakTable
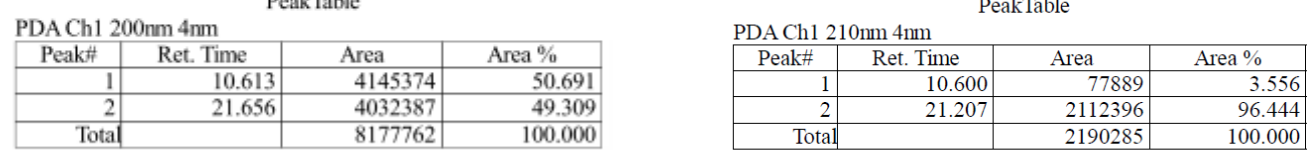

${ }^{1} \mathrm{H}$ NMR (400 MHz, $\left.\mathrm{CDCl}_{3}\right): \delta$ 7.33-7.26 (m, 2H), 7.23-7.10 (m, 3H), 4.03 (q, J=7.1 Hz, 2H), 3.13-2.92 (m, 1H), $2.63(\mathrm{dd}, J=15.0,7.1 \mathrm{~Hz}, 1 \mathrm{H}), 2.56(\mathrm{dd}, J=15.0,8.2 \mathrm{~Hz}, 1 \mathrm{H}), 1.79-1.59(\mathrm{~m}, 2 \mathrm{H}), 1.13(\mathrm{t}, J=7.1 \mathrm{~Hz}$, $3 \mathrm{H}), 0.79(\mathrm{t}, J=7.3 \mathrm{~Hz}, 3 \mathrm{H})$.

GC-MS (EI): Calcd for $\mathrm{C}_{13} \mathrm{H}_{18} \mathrm{O}_{2}[\mathrm{M}]^{+}: 206.1$ Found: 206.2.<smiles>CC(CC#N)c1ccccc1</smiles>

\section{(S)-3-Phenylbutanenitrile 2o [211230-12-5].}

(E)-3-Phenylbut-2-enenitrile (44 mg, $0.3 \mathrm{mmol}$ ) were used. The reaction completed after $24 \mathrm{~h}$ at $80{ }^{\circ} \mathrm{C}$. The product was isolated by flash chromatography $\left(\mathrm{Et}_{2} \mathrm{O} /\right.$ hexanes $\left.1: 15\right)$ as colorless oil. $40 \mathrm{mg}, 93 \%$ yield.

$[\alpha]^{23}{ }_{D}=-2.6^{\circ}\left(\mathrm{c}=0.75 \mathrm{CHCl}_{3}\right)$

Ee: 93\%. Daicel Chiralcel OD-H, n-hexane/isopropanol 90/10, flow rate $=0.5 \mathrm{~mL} / \mathrm{min}$. $\mathrm{mAU}$
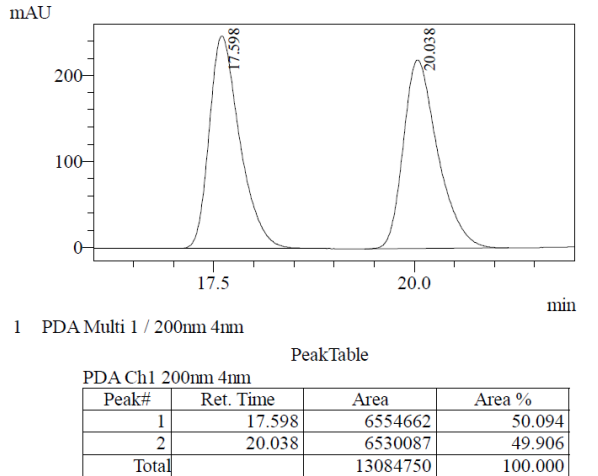

maU

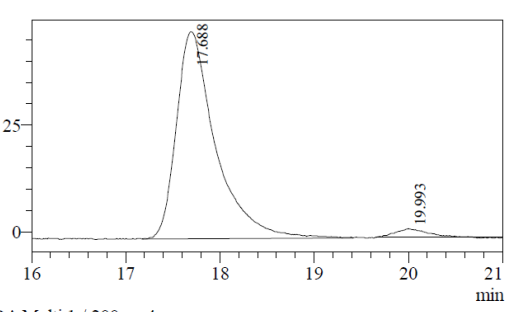

1 PDA Multi $1 / 200 \mathrm{~nm} 4 \mathrm{~nm}$

PeakTable

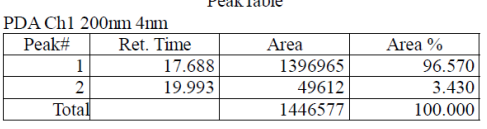

${ }^{1} \mathrm{H}$ NMR $\left(400 \mathrm{MHz}, \mathrm{CDCl}_{3}\right): \delta$ 7.38-7.32 (m, 2H), 7.30-7.23 (m, 3H), 3.22-3.11 (m, 1H), 2.67-2.50 (m, 2H), $1.46(\mathrm{~d}, J=7.0 \mathrm{~Hz}, 3 \mathrm{H})$. 
GC-MS (EI): Calcd for $\mathrm{C}_{10} \mathrm{H}_{11} \mathrm{~N}[\mathrm{M}]^{+}$: 145.1 Found: 145.1. 


\section{Asymmetric umpolung hydrogenation of $\alpha, \beta$-unsaturated ketones}

A general procedure: In an argon-filled glove box, $\mathrm{NiBr}_{2}(\mathrm{DME})(4.9 \mathrm{mg}, 0.016 \mathrm{mmol}),(R)$-Et-DuPhos (5.8 $\mathrm{mg}, 0.016 \mathrm{mmol})$, tetra- $n$-butylammonium iodide $(14.8 \mathrm{mg}, 0.04 \mathrm{mmol})$, indium powder $(0.3 \mathrm{mmol}, 34 \mathrm{mg})$ and dry $\mathrm{PhCF}_{3}(0.2 \mathrm{~mL})$ were charged into a dry $10-\mathrm{mL}$ Schlenk tube. After stirring for $15 \mathrm{~min}$ at room temperature, acetic acid ( $0.04 \mathrm{mmol}, 0.2$ equiv) in $\mathrm{H}_{2} \mathrm{O}(0.4 \mathrm{~mL}, 22.2 \mathrm{mmol}, 110$ equiv) and $\alpha, \beta$-unsaturated ketone $(0.2 \mathrm{mmol})$ were added. The reaction mixture was heated with vigorous stirring in an oil bath maintained at $80^{\circ} \mathrm{C}$ for $24 \mathrm{~h}$, until the enone was mostly consumed as monitored by GC. After the reaction mixture was cooled to room temperature, solid $\mathrm{NaHCO}_{3}$ was added to basify the mixture. After addition of silica gel and evaporation of the solvent on a rotary evaporator, the residue was dry loaded on a silica gel column and purified by flash chromatography using ethyl acetate and hexanes as eluent. The enantioselectivity (ee) of the purified product was determined by chiral HPLC analysis with Daicel Chiralcel columns.<smiles>CC(=O)CC(C)c1ccccc1</smiles>

\section{(S)-4-Phenyl-2-pentanone 4a [32587-80-7].}

The product was isolated by flash chromatography (EA/hexanes 1:50) as colorless oil. $29 \mathrm{mg}, 88 \%$ yield. $[\alpha]^{23}=+22.7^{\circ}\left(c=1.6, \mathrm{CDCl}_{3}\right) .^{4}$

Ee: $82 \%$. Daicel Chiralcel OD-H, $n$-hexane/isopropanol 99/1, flow rate $=0.5 \mathrm{~mL} / \mathrm{min}$.

mAU

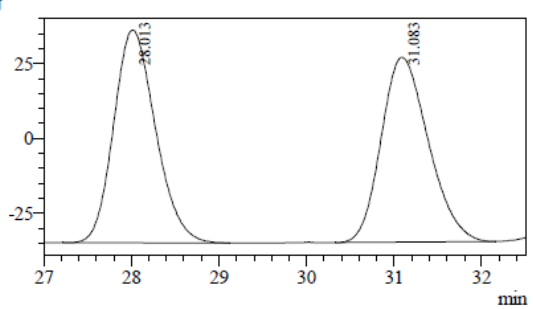

1 PDA Multi $1 / 210 \mathrm{~nm} 4 \mathrm{~nm}$

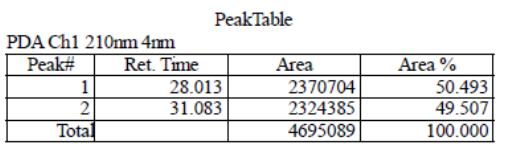

$\mathrm{mAU}$

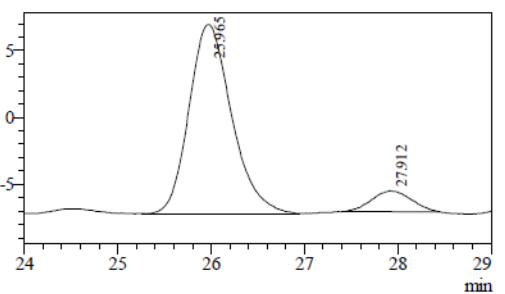

1 PDAMulti $1 / 212 \mathrm{~nm} 4 \mathrm{~nm}$

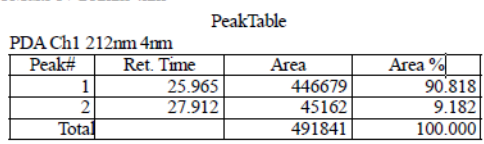

${ }^{1} \mathrm{H}$ NMR (400 MHz, $\mathrm{CDCl}_{3}$ ): $\delta$ 7.34-7.27 (m, 2H), 7.23-7.16 (m, 3H), 3.37-3.23 (m, 1H), $2.76(\mathrm{dd}, J=16.3$, $6.5 \mathrm{~Hz}, 1 \mathrm{H}), 2.66(\mathrm{dd}, J=16.3,7.9 \mathrm{~Hz}, 1 \mathrm{H}), 2.07$ (s, 3H), 1.27 (d, $J=7.0 \mathrm{~Hz}, 3 \mathrm{H})$.

${ }^{13} \mathrm{C}$ NMR (101 MHz, $\left.\mathrm{CDCl}_{3}\right): \delta 207.9,146.2,128.6,126.8,126.3,52.0,35.5,30.6,22.0$. 
GC-MS (EI): Calcd for $\mathrm{C}_{11} \mathrm{H}_{14} \mathrm{O}[\mathrm{M}]^{+}:$: 162.1. Found: 162.1 .<smiles>COc1ccc(C([14CH3])CC(C)=O)cc1</smiles>

(S)-4-(4-Methoxyphenyl)-2-pentanone 4b [162424-49-9].

The product was isolated by flash chromatography (EA/hexanes 1:50) as colorless oil. $38 \mathrm{mg}, 86 \%$ yield. $[\alpha]^{23}{ }_{\mathrm{D}}=+25.9^{\circ}\left(c=1.3, \mathrm{CDCl}_{3}\right)$.

Ee: $84 \%$. Daicel Chiralcel IC, $n$-hexane/isopropanol 90/10, flow rate $=0.5 \mathrm{~mL} / \mathrm{min}$.
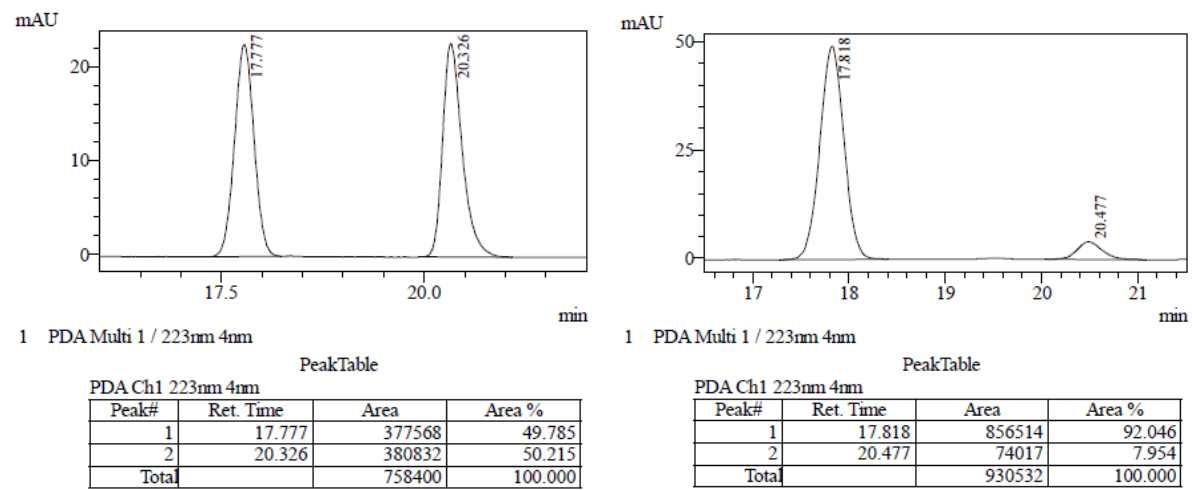

1 PDA Multi $1 / 223 \mathrm{~nm} 4 \mathrm{~nm}$

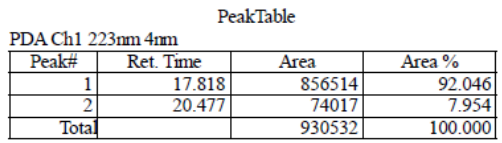

${ }^{1} \mathrm{H}$ NMR (400 MHz, $\left.\mathrm{CDCl}_{3}\right): \delta$ 7.21-7.08 (m, 2H), 6.94-6.73 (m, 2H), $3.78(\mathrm{~s}, 3 \mathrm{H}), 3.42-3.18(\mathrm{~m}, 1 \mathrm{H}), 2.72$ (dd, $J=16.0,6.8 \mathrm{~Hz}, 1 \mathrm{H}), 2.62(\mathrm{dd}, J=16.1,7.7 \mathrm{~Hz}, 1 \mathrm{H}), 2.05(\mathrm{~s}, 3 \mathrm{H}), 1.24(\mathrm{~d}, J=7.0 \mathrm{~Hz}, 3 \mathrm{H})$.

${ }^{13} \mathrm{C}$ NMR (101 MHz, $\left.\mathrm{CDCl}_{3}\right): \delta 208.2,158.2,138.4,127.8,114.1,55.4,52.4,34.9,30.7,22.4$. GC-MS (EI): Calcd for $\mathrm{C}_{12} \mathrm{H}_{16} \mathrm{O}_{2}[\mathrm{M}]^{+}$: 192.1. Found: 192.1 .<smiles>CC(=O)CC(C)c1ccc(C(F)(F)F)cc1</smiles>

(S)-4-p-Trifluoromethylphenyl-2-pentanone 4c [652994-31-5].

The product was isolated by flash chromatography (EA/hexanes 1:50) as colorless oil. $37 \mathrm{mg}, 80 \%$ yield. $[\alpha]^{23}{ }_{\mathrm{D}}=+9.36^{\circ}\left(c=0.86, \mathrm{CDCl}_{3}\right)$.

Ee: $82 \%$. Daicel Chiralcel OJ-H, $n$-hexane/isopropanol 99.5/0.5, flow rate $=0.5 \mathrm{~mL} / \mathrm{min}$. 

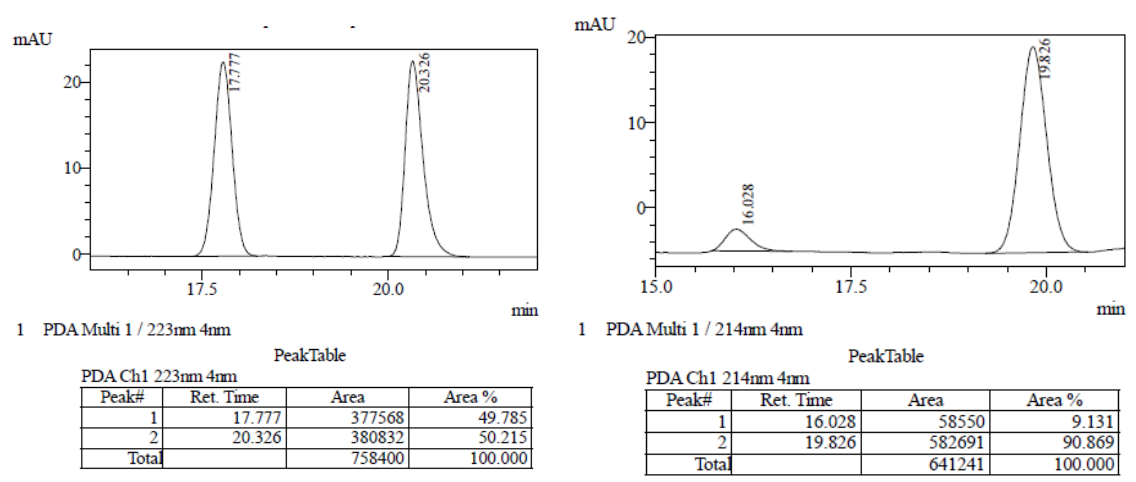

${ }^{1} \mathrm{H}$ NMR $\left(400 \mathrm{MHz}, \mathrm{CDCl}_{3}\right): \delta 7.54(\mathrm{~d}, J=8.2 \mathrm{~Hz}, 2 \mathrm{H}), 7.32(\mathrm{~d}, J=8.1 \mathrm{~Hz}, 2 \mathrm{H}), 3.34-3.31(\mathrm{~m}, 1 \mathrm{H}), 2.77(\mathrm{dd}$, $J=16.8,6.8 \mathrm{~Hz}, 1 \mathrm{H}), 2.69(\mathrm{dd}, J=16.8,7.5 \mathrm{~Hz}, 1 \mathrm{H}), 2.08(\mathrm{~s}, 3 \mathrm{H}), 1.27(\mathrm{~d}, J=7.0 \mathrm{~Hz}, 3 \mathrm{H})$.

${ }^{13} \mathrm{C}$ NMR $\left(101 \mathrm{MHz}, \mathrm{CDCl}_{3}\right): \delta 207.0,150.3,128.6\left(\mathrm{q}, J_{C-F}=32.2 \mathrm{~Hz}\right), 127.2,125.5\left(\mathrm{q}, J_{C-F}=3.8 \mathrm{~Hz}\right), 124.2$ $\left(\mathrm{q}, J_{C-F}=276.8 \mathrm{~Hz}\right), 51.5,35.1,30.5,21.8$.

${ }^{19} \mathrm{~F}$ NMR (101 MHz, $\left.\mathrm{CDCl}_{3}\right): \delta-62.4$.

GC-MS (EI): Calcd for $\mathrm{C}_{12} \mathrm{H}_{13} \mathrm{~F}_{3} \mathrm{O}[\mathrm{M}]^{+}: 230.1$ Found: 230.2 .<smiles>CC(=O)CC(C)c1ccc2ccccc2c1</smiles>

\section{(S)-4-ß-naphthyl-2-pentanone 4d [183615-97-6].}

The product was isolated by flash chromatography $\left(\mathrm{Et}_{2} \mathrm{O} /\right.$ hexanes $\left.1: 10\right)$ as colorless oil. $37 \mathrm{mg}, 87 \%$ yield. $[\alpha]^{23}=+30.3^{\circ}\left(c=1.1, \mathrm{CDCl}_{3}\right)$.

Ee: $80 \%$. Daicel Chiralcel OJ-H, $n$-hexane/isopropanol 90/10, flow rate $=0.5 \mathrm{~mL} / \mathrm{min}$.
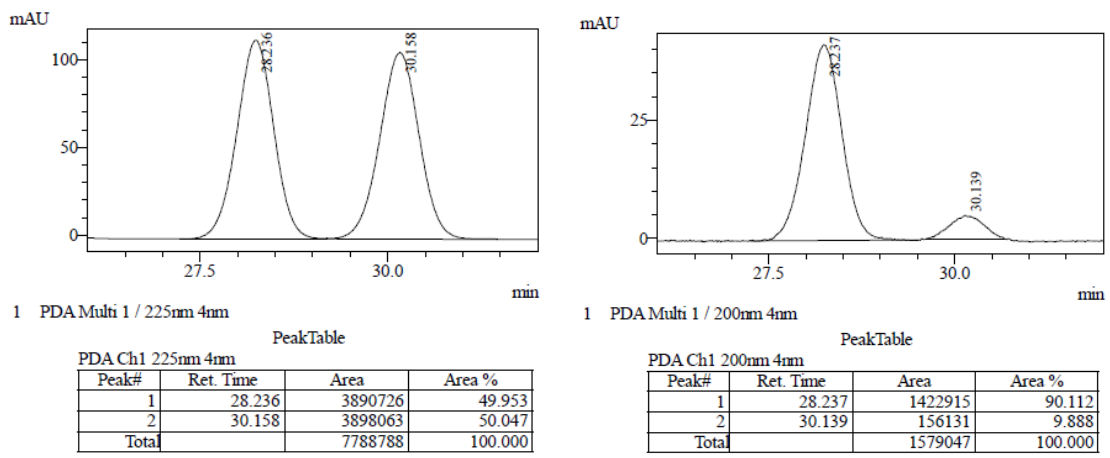

${ }^{1} \mathrm{H}$ NMR (400 MHz, $\left.\mathrm{CDCl}_{3}\right): \delta$ 7.84-7.74 (m, 3H), $7.64(\mathrm{~s}, 1 \mathrm{H})$, 7.50-7.41 (m, 2H), 7.38-7.33 (m, 1H), 3.56$3.39(\mathrm{~m}, 1 \mathrm{H}), 2.87(\mathrm{dd}, J=16.3,6.5 \mathrm{~Hz}, 1 \mathrm{H}), 2.75(\mathrm{dd}, J=16.3,7.8 \mathrm{~Hz}, 1 \mathrm{H}), 2.08(\mathrm{~s}, 3 \mathrm{H}), 1.36(\mathrm{~d}, J=6.9$ $\mathrm{Hz}, 3 \mathrm{H})$.

${ }^{13} \mathrm{C}$ NMR $\left(101 \mathrm{MHz}, \mathrm{CDCl}_{3}\right): \delta 207.9,143.7,133.7,132.4,128.4,127.76,127.72,126.2,125.7,125.5$, $125.1,52.1,35.7,30.8,22.1$. 
<smiles>CC(=O)CC(C)c1ccc2c(c1)OCO2</smiles>

\section{(S)-4-(1,3-Benzodioxol-5-yl)-2-pentanone 4e [86347-18-4].}

The product was isolated by flash chromatography $\left(\mathrm{Et}_{2} \mathrm{O} /\right.$ hexanes $\left.1: 10\right)$ as colorless oil. $36 \mathrm{mg}, 88 \%$ yield. $[\alpha]^{23}{ }_{\mathrm{D}}=+26.2^{\circ}\left(c=1.4, \mathrm{CDCl}_{3}\right)$.

Ee: $86 \%$. Daicel Chiralcel IC, $n$-hexane/isopropanol 90/10, flow rate $=0.5 \mathrm{~mL} / \mathrm{min}$.

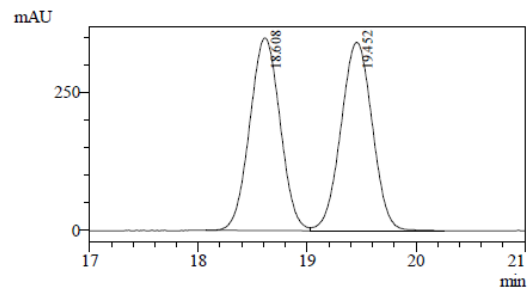

1 PDA Multi $1 / 200 \mathrm{~nm} 4 \mathrm{~nm}$

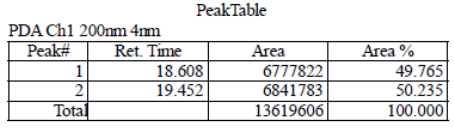
$\mathrm{mAU}$

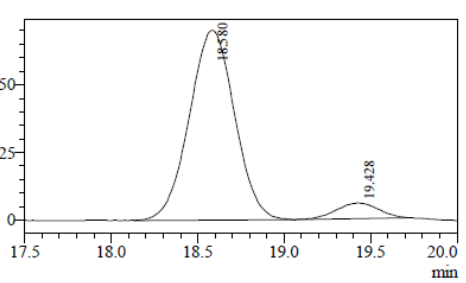

PDAMulti $1 / 205 \mathrm{~nm} 4 \mathrm{~nm}$

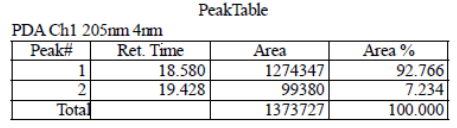

${ }^{1} \mathrm{H}$ NMR (400 MHz, $\left.\mathrm{CDCl}_{3}\right): \delta$ 6.76-6.69 (m, 2H), 6.68-6.62 (m, 1H), $5.92(\mathrm{~s}, 2 \mathrm{H}), 3.29-3.18(\mathrm{~m}, 1 \mathrm{H}), 2.70$ (dd, $J=16.2,6.8 \mathrm{~Hz}, 1 \mathrm{H}), 2.60(\mathrm{dd}, J=16.2,7.6 \mathrm{~Hz}, 1 \mathrm{H}), 2.06(\mathrm{~s}, 3 \mathrm{H}), 1.22(\mathrm{~d}, J=7.0 \mathrm{~Hz}, 3 \mathrm{H})$.

${ }^{13} \mathrm{C}$ NMR (101 MHz, $\left.\mathrm{CDCl}_{3}\right): \delta 207.9,147.8,146.0,140.3,119.8,108.4,107.3,101.0,52.3,35.4,30.7,22.4$. GC-MS (EI): Calcd for $\mathrm{C}_{12} \mathrm{H}_{14} \mathrm{O}_{3}[\mathrm{M}]^{+}:$206.1. Found: 206.1.<smiles>CCC(=O)CC(C)c1ccccc1</smiles>

(S)-5-Phenyl-3-hexanone 4f [123624-44-2].

The product was isolated by flash chromatography $\left(\mathrm{Et}_{2} \mathrm{O} /\right.$ hexanes $\left.1: 10\right)$ as colorless oil. $28.2 \mathrm{mg}, 80 \%$ yield. $[\alpha]^{23}=+32.2^{\circ}\left(c=0.53, \mathrm{CDCl}_{3}\right)$.

Ee: $80 \%$. Daicel Chiralcel OD-H, $n$-hexane/isopropanol 99/1, flow rate $=0.5 \mathrm{~mL} / \mathrm{min}$. 

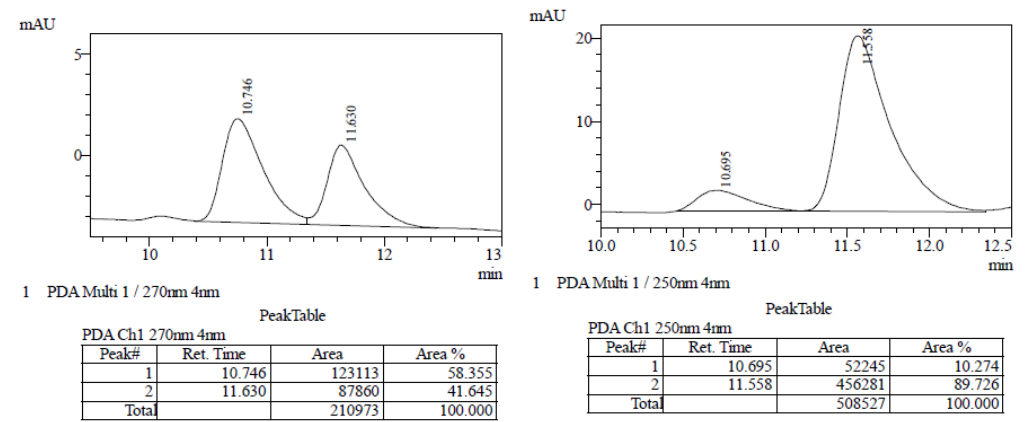

${ }^{1} \mathrm{H}$ NMR (400 MHz, $\left.\mathrm{CDCl}_{3}\right): \delta$ 7.35-7.26 (m, 2H), 7.26-7.17 (m, 3H), 3.41-3.28 (m, 1H), 2.80 (dd, $J=16.1$, $6.6 \mathrm{~Hz}, 1 \mathrm{H}), 2.65(\mathrm{dd}, J=16.0,7.8 \mathrm{~Hz}, 1 \mathrm{H}), 1.29(\mathrm{~d}, J=7.0 \mathrm{~Hz}, 3 \mathrm{H}), 1.01(\mathrm{t}, J=7.3 \mathrm{~Hz}, 3 \mathrm{H})$.

${ }^{13} \mathrm{C}$ NMR $\left(101 \mathrm{MHz}, \mathrm{CDCl}_{3}\right): \delta 210.5,146.3,128.5,126.8,126.3,50.8,36.7,35.5,22.0,7.6$.

GC-MS (EI): Calcd for $\mathrm{C}_{12} \mathrm{H}_{16} \mathrm{O}[\mathrm{M}]^{+}:$176.1. Found: 176.1 .<smiles>CC(CC(=O)c1ccccc1)c1ccccc1</smiles>

\section{(S)-1,3-Diphenyl-1-butanone 4g [20698-96-8].}

The product was isolated by flash chromatography $\left(\mathrm{Et}_{2} \mathrm{O} /\right.$ hexanes $\left.1: 10\right)$ as colorless oil. $34 \mathrm{mg}, 76 \%$ yield. $[\alpha]^{23}=+0.33^{\circ}\left(c=1.7, \mathrm{CDCl}_{3}\right)$.

Ee: $43 \%$. Daicel Chiralcel IC, $n$-hexane/isopropanol 98/2, flow rate $=0.5 \mathrm{~mL} / \mathrm{min}$.

maU

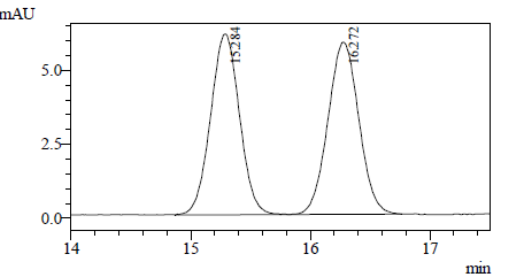

1. PDA Multi $1 / 238 \mathrm{rm} 4 \mathrm{~nm}$

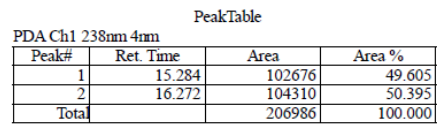

$\mathrm{mAU}$

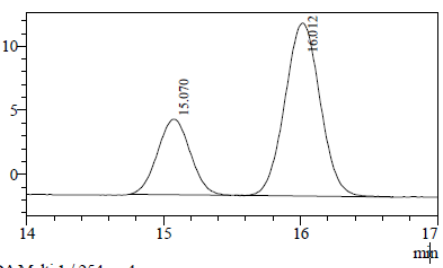

PDAMulti $1 / 254 \mathrm{rm} 4 \mathrm{~nm} \quad$ PeakTable

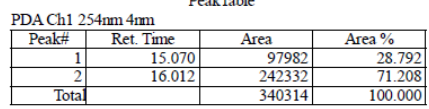

${ }^{1} \mathrm{H}$ NMR (400 MHz, $\left.\mathrm{CDCl}_{3}\right): \delta$ 7.97-7.88 (m, 2H), 7.62-7.50 (m, 1H), 7.51-7.39 (m, 2H), 7.37-7.25 (m, 4H), 7.23-7.13 (m, 1H), 3.57-3.43 (m, 1H), $3.30(\mathrm{dd}, J=16.4,5.7 \mathrm{~Hz}, 1 \mathrm{H}), 3.19$ (dd, $J=16.5,8.3 \mathrm{~Hz}, 1 \mathrm{H}), 1.34(\mathrm{~d}$, $J=6.9 \mathrm{~Hz}, 3 \mathrm{H})$.

${ }^{13} \mathrm{C}$ NMR (101 MHz, $\left.\mathrm{CDCl}_{3}\right): \delta 199.1,133.0,128.6,128.1,126.9,126.3,47.0,35.6,21.9$.

GC-MS (EI): Calcd for $\mathrm{C}_{16} \mathrm{H}_{16} \mathrm{O}[\mathrm{M}]^{+}:$224.1. Found: 224.1 . 


\section{Asymmetric Umpolung hydrogenation of allylic alcohols}

A procedure: In an argon-filled glove box, $\mathrm{NiBr}_{2}(\mathrm{DME})(1.8 \mathrm{mg}, 0.006 \mathrm{mmol}),(R)-\mathrm{Me}-\mathrm{DuPhos}(1.8 \mathrm{mg}$, $0.006 \mathrm{mmol})$, and dry THF $(0.6 \mathrm{~mL})$ were charged into a dry 10-mL Schlenk tube. After stirring for $15 \mathrm{~min}$ at room temperature, tetra- $n$-butylammonium iodide $(14.8 \mathrm{mg}, 0.04 \mathrm{mmol})$, indium powder (1.5 equiv, 0.3 mmol, $34 \mathrm{mg})$, 2-butenol ( $0.2 \mathrm{mmol})$, water ( $0.6 \mathrm{mmol}, 3$ equiv) and acetic acid ( $35 \mu \mathrm{L}, 0.6 \mathrm{mmol}, 3$ equiv) were added. The reaction mixture was heated with vigorous stirring in an oil bath maintained at $80{ }^{\circ} \mathrm{C}$, until the enol was mostly consumed as monitored by GC. After the reaction mixture was cooled to room temperature, solid $\mathrm{NaHCO}_{3}$ was added to basify the mixture. After addition of silica gel and evaporation of the solvent on a rotary evaporator, the residue was dry loaded on a silica gel column and purified by flash chromatography using ethyl acetate and hexanes as eluent. The enantioselectivity (ee) of the purified product was determined by chiral HPLC analysis with Daicel Chiralcel columns.<smiles>O=[N+]([O-])CCO</smiles>

\section{(S)-3-Methyl-3-phenylpropanol 6a [2031-46-1].}

The product was isolated by flash chromatography $\left(\mathrm{Et}_{2} \mathrm{O} /\right.$ hexanes $\left.1: 10\right)$ as colorless oil. $29 \mathrm{mg}, 96 \%$ yield. $[\alpha]^{23}=+17.7^{\circ}\left(c=1.97, \mathrm{CDCl}_{3}\right) .^{3}$

Ee: $89 \%$. Daicel Chiralcel IC, $n$-hexane/isopropanol 95/5, flow rate $=0.5 \mathrm{~mL} / \mathrm{min}$.
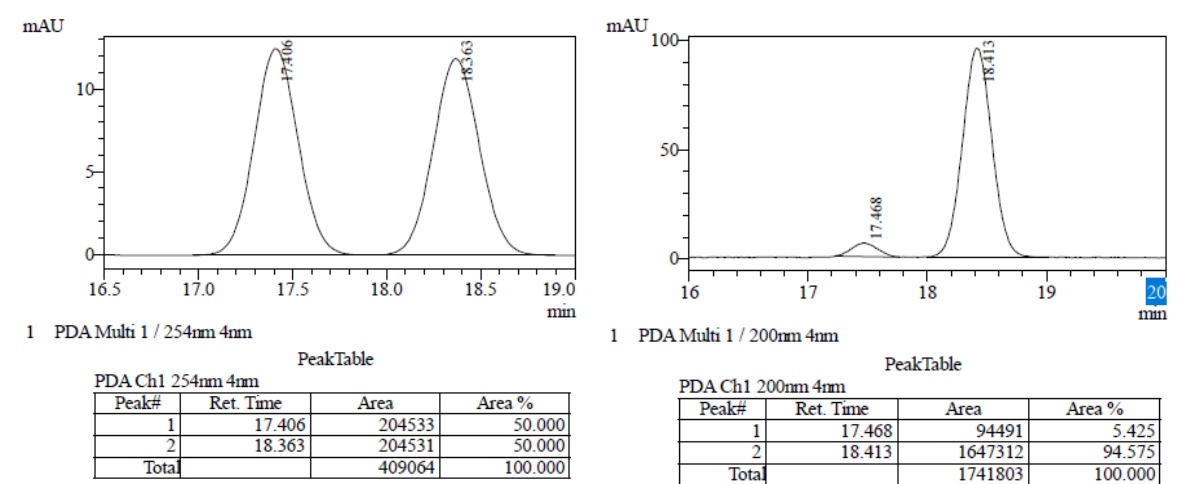

1 PDA Multi $1 / 200 \mathrm{~nm} 4 \mathrm{~nm}$

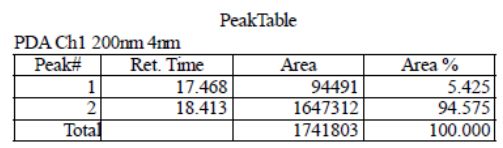

${ }^{1} \mathrm{H}$ NMR (400 MHz, $\left.\mathrm{CDCl}_{3}\right): \delta$ 7.35-7.27 (m, 2H), 7.25-7.15 (m, 3H), 3.62-3.48 (m, 2H), 2.95-2.83 (m, 1H), 1.90-1.79 (m, 2H), 1.71 (br s, OH), 1.29 (d, $J=7.0 \mathrm{~Hz}, 3 \mathrm{H})$.

${ }^{13} \mathrm{C}$ NMR $\left(101 \mathrm{MHz}, \mathrm{CDCl}_{3}\right): \delta 146.9,128.5,127.0,126.1,61.1,41.0,36.5,22.4$.

GC-MS (EI): Calcd for $\mathrm{C}_{10} \mathrm{H}_{14} \mathrm{O}[\mathrm{M}]^{+}: 150.1$ Found: 150.1 . 
<smiles>COc1ccc(C(C)CCO)cc1</smiles>

(S)-3-Methyl-3-p-anisylpropanol 6b [1333411-76-9].

The product was isolated by flash chromatography ( $\mathrm{Et}_{2} \mathrm{O} /$ hexanes $\left.1: 10\right)$ as colorless oil. $34 \mathrm{mg}, 94 \%$ yield. $[\alpha]^{23}=+16.8^{\circ}\left(c=1.84, \mathrm{CDCl}_{3}\right)$.

Ee: $85 \%$. Daicel Chiralcel OD-H, $n$-hexane/isopropanol 99/1, flow rate $=0.5 \mathrm{~mL} / \mathrm{min}$.
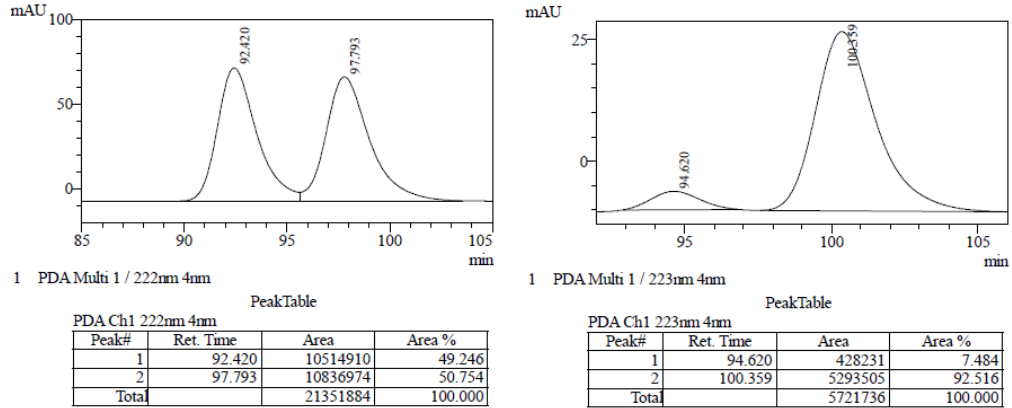

${ }^{1} \mathrm{H}$ NMR (400 MHz, $\left.\mathrm{CDCl}_{3}\right): \delta 7.12(\mathrm{~d}, J=8.6 \mathrm{~Hz}, 2 \mathrm{H}), 6.85$ (d, $\left.J=8.6 \mathrm{~Hz}, 2 \mathrm{H}\right), 3.79$ (s, 3H), 3.62-3.48 (m, 2H), 2.91-2.76 (m, 1H), 1.93-1.74 (m, 2H), 1.29 (d, $J=7.0 \mathrm{~Hz}, 3 \mathrm{H}), 1.20$ (br s, OH).

${ }^{13} \mathrm{C} \mathrm{NMR}\left(101 \mathrm{MHz}, \mathrm{CDCl}_{3}\right): \delta 157.9,138.9,127.8,113.9,61.3,55.2,41.1,35.7,22.6$.

GC-MS (EI): Calcd for $\mathrm{C}_{10} \mathrm{H}_{14} \mathrm{O}[\mathrm{M}]^{+}: 180.1$ Found: 180.0 .<smiles>OCC[CH]c1ccc(C(F)(F)F)cc1</smiles>

(S)-3-Methyl-3-p-trifluorophenylpropanol 6c [1225551-50-7]

The product was isolated by flash chromatography $\left(\mathrm{Et}_{2} \mathrm{O} /\right.$ hexanes $\left.1: 10\right)$ as colorless oil. $41 \mathrm{mg}, 93 \%$ yield. $[\alpha]^{23}{ }_{\mathrm{D}}=+12.1^{\circ}\left(c=1.0, \mathrm{CDCl}_{3}\right)$.

Ee: $89 \%$. Daicel Chiralcel OD-H, $n$-hexane/isopropanol 98/2, flow rate $=0.5 \mathrm{~mL} / \mathrm{min}$.
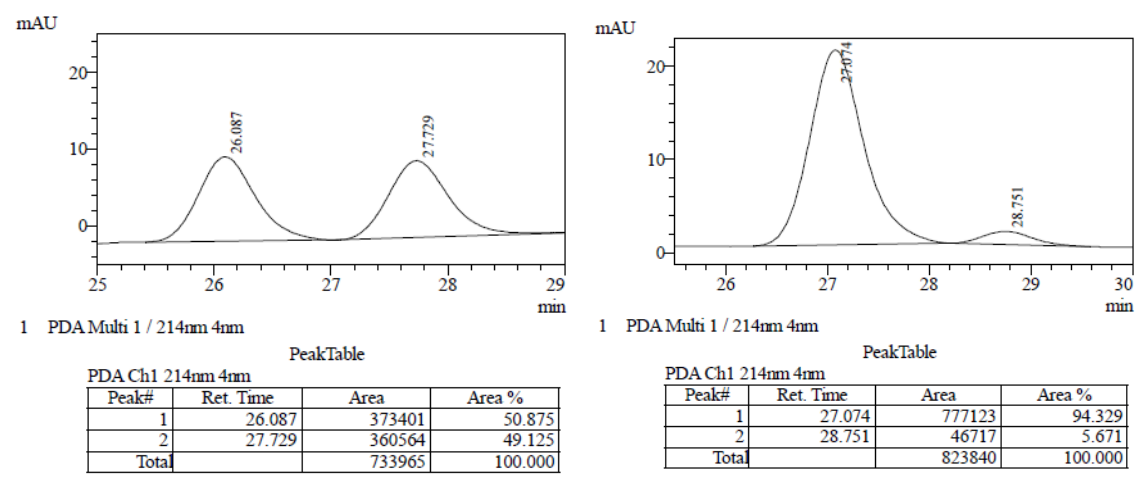
${ }^{1} \mathrm{H}$ NMR (400 MHz, $\left.\mathrm{CDCl}_{3}\right): \delta 7.55$ (d, $\left.J=8.2 \mathrm{~Hz}, 2 \mathrm{H}\right), 7.32(\mathrm{~d}, J=8.1 \mathrm{~Hz}, 2 \mathrm{H}), 3.74-3.44(\mathrm{~m}, 2 \mathrm{H}), 3.10-$ $2.88(\mathrm{~m}, 1 \mathrm{H}), 2.00-1.73(\mathrm{~m}, 2 \mathrm{H}), 1.29(\mathrm{~d}, J=7.0 \mathrm{~Hz}, 3 \mathrm{H}), 1.23$ (br s, OH).

${ }^{13} \mathrm{C}$ NMR $\left(101 \mathrm{MHz}, \mathrm{CDCl}_{3}\right): \delta 151.0,127.7\left(\mathrm{q}, J_{C-F}=32.3 \mathrm{~Hz}\right), 127.3,125.4\left(\mathrm{q}, J_{C-F}=2.6 \mathrm{~Hz}\right), 124.0\left(\mathrm{q}, J_{C-}\right.$ $\left.{ }_{F}=185.7 \mathrm{~Hz}\right), 60.8,40.7,36.2,22.1$.

${ }^{19} \mathrm{~F}$ NMR $\left(101 \mathrm{MHz}, \mathrm{CDCl}_{3}\right): \delta-62.3$.

GC-MS (EI): Calcd for $\mathrm{C}_{11} \mathrm{H}_{13} \mathrm{~F}_{3} \mathrm{O}[\mathrm{M}]^{+}: 218.1$ Found: 218.0.<smiles>CC(CCO)c1ccc2ccccc2c1</smiles>

(S)-3-Methyl-3-ß-naphthylpropanol 6d [1607017-52-6].

The product was isolated by flash chromatography $\left(\mathrm{Et}_{2} \mathrm{O} /\right.$ hexanes $\left.1: 10\right)$ as colorless oil. $37 \mathrm{mg}, 92 \%$ yield. $[\alpha]^{23}=+20.2^{\circ}\left(c=1.6, \mathrm{CDCl}_{3}\right)$.

Ee: $88 \%$. Daicel Chiralcel OD-H, $n$-hexane/isopropanol 90/10, flow rate $=0.5 \mathrm{~mL} / \mathrm{min}$. $\mathrm{mAU}$

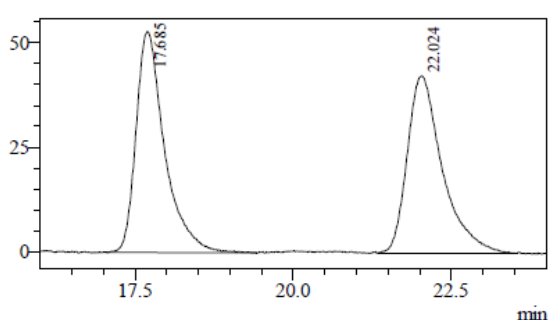

1 PDA Multi $1 / 200 \mathrm{~nm} 4 \mathrm{~nm}$

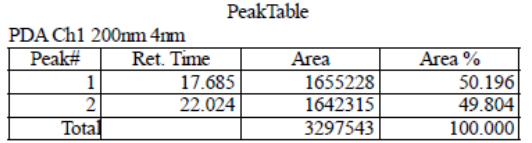

mAU

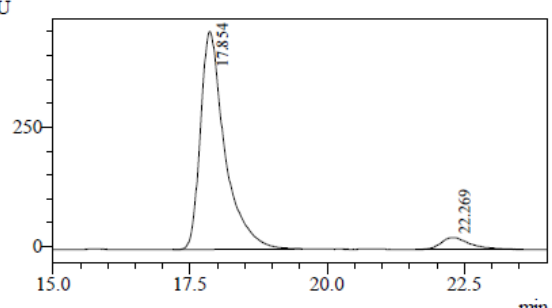

1 PDA Multi $1 / 200 \mathrm{~nm} 4 \mathrm{~nm}$

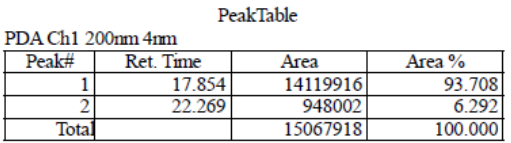

${ }^{1} \mathrm{H}$ NMR $\left(400 \mathrm{MHz}, \mathrm{CDCl}_{3}\right): \delta$ 7.85-7.74 (m, 3H), $7.64(\mathrm{~s}, 1 \mathrm{H}), 7.50-7.30(\mathrm{~m}, 3 \mathrm{H}), 3.69-3.51(\mathrm{~m}, 2 \mathrm{H}), 3.13-$ $2.99(\mathrm{~m}, 1 \mathrm{H}), 2.03-1.89(\mathrm{~m}, 2 \mathrm{H}), 1.37$ (d, $J=7.0 \mathrm{~Hz}, 3 \mathrm{H}), 1.20$ (br s, OH).

${ }^{13} \mathrm{C}$ NMR $\left(101 \mathrm{MHz}, \mathrm{CDCl}_{3}\right): \delta 144.3,133.6,132.3,128.2,127.6,127.5,126.0,125.6,125.2,125.3,61.3$, $40.8,36.6,22.5$.

GC-MS (EI): Calcd for $\mathrm{C}_{14} \mathrm{H}_{16} \mathrm{O}[\mathrm{M}]^{+}: 200.1$ Found: 200.1 .<smiles>OCC[CH]c1ccc2c(c1)OCO2</smiles>

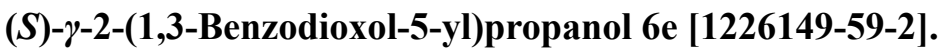

The product was isolated by flash chromatography $\left(\mathrm{Et}_{2} \mathrm{O} /\right.$ hexanes $\left.1: 10\right)$ as colorless oil. $36 \mathrm{mg}, 93 \%$ yield. 
$[\alpha]^{23}{ }_{D}=14.9^{\circ}\left(c=1.9, \mathrm{CDCl}_{3}\right)$.

Ee: $87 \%$. Daicel Chiralcel OJ-H, $n$-hexane/isopropanol 97/3, flow rate $=0.5 \mathrm{~mL} / \mathrm{min}$.

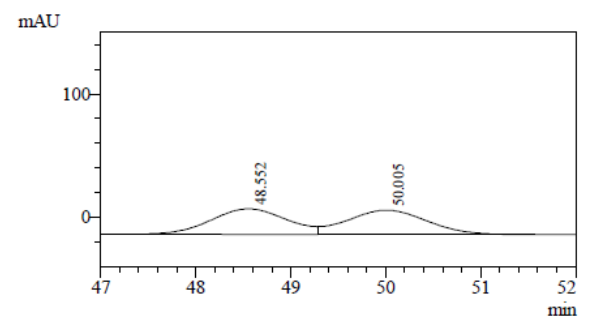

1 PDA Multi $1 / 220 \mathrm{~nm} 4 \mathrm{~nm}$
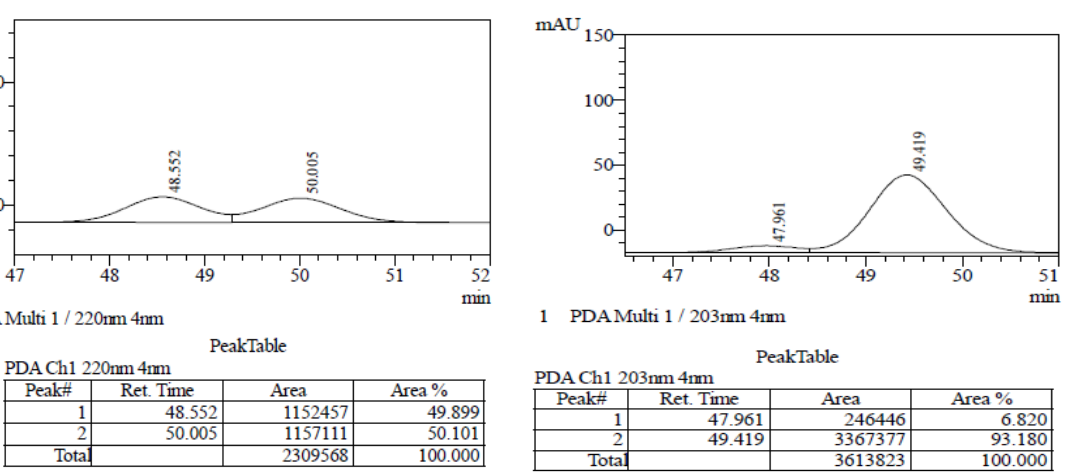

${ }^{1} \mathrm{H}$ NMR (400 MHz, $\left.\mathrm{CDCl}_{3}\right): \delta$ 6.76-6.67 (m, 2H), 6.67-6.62 (m, 1H), $5.92(\mathrm{~s}, 2 \mathrm{H}), 3.65-3.47(\mathrm{~m}, 2 \mathrm{H}), 2.87-$ $2.75(\mathrm{~m}, 1 \mathrm{H}), 1.90-1.69(\mathrm{~m}, 2 \mathrm{H}), 1.23(\mathrm{~d}, J=7.0 \mathrm{~Hz}, 3 \mathrm{H}), 1.20(\mathrm{br} \mathrm{s}, \mathrm{OH})$.

${ }^{13} \mathrm{C}$ NMR (101 MHz, $\left.\mathrm{CDCl}_{3}\right): \delta$ 147.7, 145.8, 140.8, 119.9, 108.2, 107.2, 100.8, 61.2, 41.1, 39.3, 22.7

GC-MS (EI): Calcd for $\mathrm{C}_{11} \mathrm{H}_{14} \mathrm{O}_{3}[\mathrm{M}]^{+}$: 194.1 Found: 194.1 .

\section{Asymmetric umpolung deuteration of $\alpha, \beta$-unsaturated esters}

A general procedure: In an argon-filled glove box, $\mathrm{NiBr}_{2}(\mathrm{DME})(1.8 \mathrm{mg}, 0.006 \mathrm{mmol}),(R)-\mathrm{Me}-\mathrm{DuPhos}(1.8$ $\mathrm{mg}, 0.006 \mathrm{mmol})$, tetra- $n$-butylammonium iodide (14.8 $\mathrm{mg}, 0.04 \mathrm{mmol})$, indium powder $(25.2 \mathrm{mg}, 0.22$ $\mathrm{mmol})$ and dry $\mathrm{PhCF}_{3}(0.6 \mathrm{~mL})$ were charged into a dry 10-mL Schlenk tube. After stirring for $15 \mathrm{~min}$ at room temperature, acetic acid ( 0.2 equiv, $0.04 \mathrm{mmol})$ in $\mathrm{D}_{2} \mathrm{O}(0.4 \mathrm{~mL}, 20 \mathrm{mmol}, 100$ equiv) and olefin $(0.2$ mmol) were added. The reaction mixture was heated with vigorous stirring in an oil bath maintained at 80 ${ }^{\circ} \mathrm{C}$, until the olefin was mostly consumed as monitored by GC. After the reaction mixture was cooled to room temperature, solid $\mathrm{NaHCO}_{3}$ was added to basify the mixture. After addition of silica gel and evaporation of the solvent on a rotary evaporator, the residue was dry loaded on a silica gel column and purified by flash chromatography using ethyl acetate and hexanes as eluent. The enantioselectivity of the purified product was determined by chiral HPLC analysis with Daicel Chiralcel columns. The content of deuterium was calculated by comparing the integration of proton signals of nondeuteriated samples on qualitative NMR spectra.

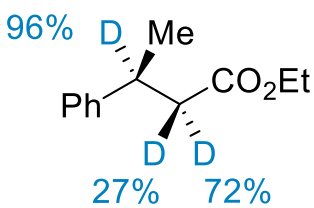




\section{(S)-Ethyl-3-phenylbutanoate-2,3- $d_{2}$ 2ad [1800576-15-1].}

The product was isolated by flash chromatography $\left(\mathrm{Et}_{2} \mathrm{O} /\right.$ hexanes $\left.1: 20\right)$ as colorless oil. $37 \mathrm{mg}, 95 \%$ yield. $[\alpha]^{23}=+24.3^{\circ}\left(c=0.23, \mathrm{CDCl}_{3}\right)$.

92\% ee. Daicel Chiralcel OD-H, $n$-hexane/isopropanol 98/2, flow rate $=0.5 \mathrm{~mL} / \mathrm{min}$.
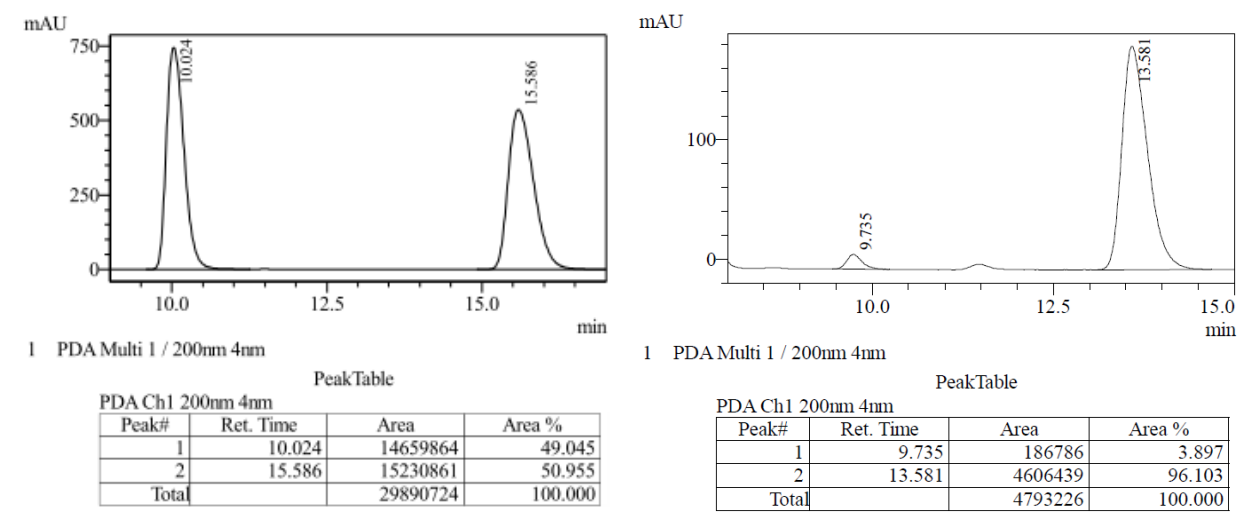

1 PDA Multi $1 / 200 \mathrm{~nm} 4 \mathrm{~nm}$

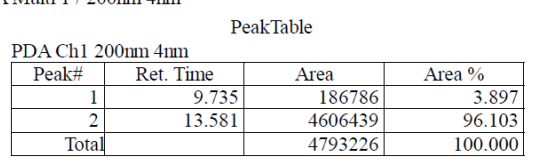

${ }^{1} \mathrm{H}$ NMR (500 MHz, $\left.\mathrm{CDCl}_{3}\right): \delta$ 7.34-7.26 (m, 2H), 7.26-7.13 (m, 3H), 4.19-4.01 (m, 2H), 3.33-3.14 (m,

$0.04 \mathrm{H}), 2.59(\mathrm{~s}, 0.28 \mathrm{H}), 2.52(\mathrm{~s}, 0.73 \mathrm{H}), 1.30(\mathrm{~s}, 3 \mathrm{H}), 1.18(\mathrm{t}, J=7.1 \mathrm{~Hz}, 3 \mathrm{H})$.

${ }^{2} \mathrm{H}$ NMR (61 MHz, $\left.\mathrm{CDCl}_{3}\right): \delta 3.27,2.61,2.55$.

${ }^{13} \mathrm{C} \mathrm{NMR}\left(126 \mathrm{MHz}, \mathrm{CDCl}_{3}\right): \delta 172.5,145.7,128.5,126.8,126.5,60.1,42.6(\mathrm{t}, J=19.8 \mathrm{~Hz}), 36.1(\mathrm{t}, J=$ $20.2 \mathrm{~Hz}), 21.7(\mathrm{~d}, J=6.1 \mathrm{~Hz}), 14.18$.

LC-MS (ESI): Calcd for $\mathrm{C}_{12} \mathrm{H}_{15} \mathrm{D}_{2} \mathrm{O}_{2}^{+}[\mathrm{M}+\mathrm{H}]^{+}$: 195.1. Found: 195.1 .

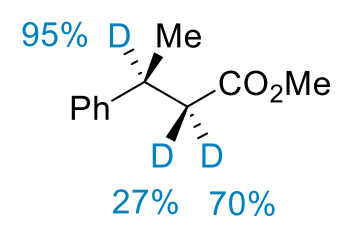

(S)-Methyl 3-phenylbutanoate-2,3- $d_{2} 2$ bd.

The product was isolated by flash chromatography $\left(\mathrm{Et}_{2} \mathrm{O} /\right.$ hexanes 1:20) as colorless oil. $34 \mathrm{mg}, 95 \%$ yield. $[\alpha]^{23}{ }_{\mathrm{D}}=+20.3^{\circ}\left(c=0.8, \mathrm{CDCl}_{3}\right)$.

Ee: $93 \%$. Daicel Chiralcel OD-H, $n$-hexane/isopropanol 98/2, flow rate $=0.5 \mathrm{~mL} / \mathrm{min}$. 

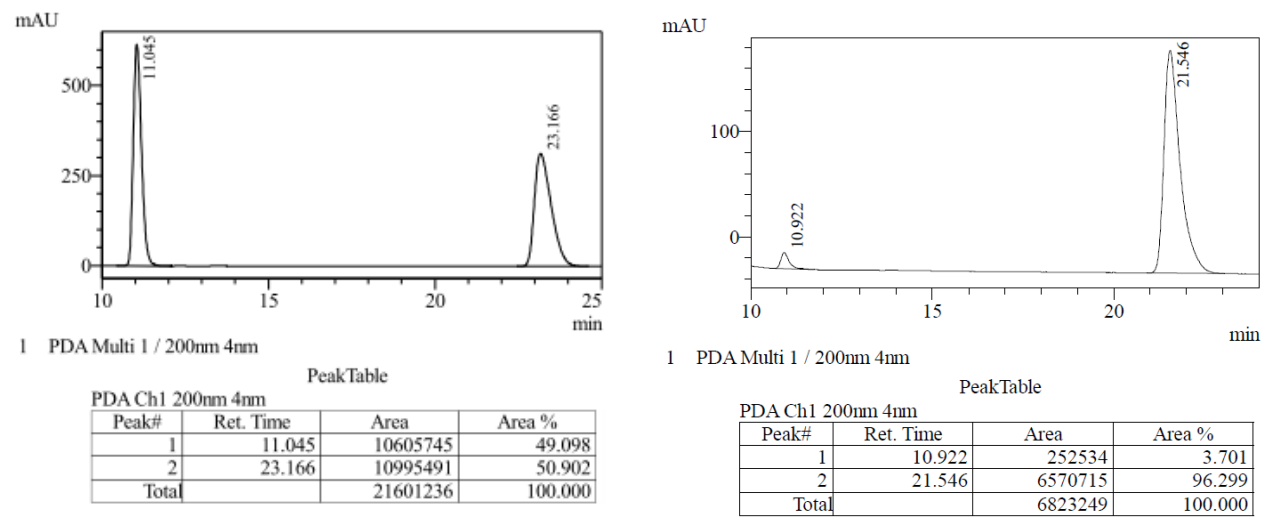

${ }^{1} \mathrm{H}$ NMR (400 MHz, $\left.\mathrm{CDCl}_{3}\right): \delta$ 7.34-7.27 (m, 2H), 7.25-7.17 (m, 3H), $3.62(\mathrm{~s}, 3 \mathrm{H}), 3.31-3.22(\mathrm{~m}, 0.05 \mathrm{H}), 2.60$ (s, 0.30), $2.53(\mathrm{~s}, 0.73), 1.29(\mathrm{~s}, 3 \mathrm{H})$.

${ }^{2} \mathrm{H}$ NMR $\left(61 \mathrm{MHz}, \mathrm{CDCl}_{3}\right): \delta 3.27,2.63,2.55$.

${ }^{13} \mathrm{C}$ NMR $\left(101 \mathrm{MHz}, \mathrm{CDCl}_{3}\right): \delta 172.9,145.7,128.5,126.7,126.4,51.5,42.4(\mathrm{t}, J=19.8 \mathrm{~Hz}), 36.0(\mathrm{t}, J=$ $20.0 \mathrm{~Hz}), 21.6(\mathrm{~d}, J=4.8 \mathrm{~Hz})$.

LC-MS (ESI): Calcd for $\mathrm{C}_{11} \mathrm{H}_{13} \mathrm{D}_{2} \mathrm{O}_{2}^{+}[\mathrm{M}+\mathrm{H}]^{+}$: 181.1. Found: 181.0.<smiles>[2H]C([2H])(C(=O)OCC)C([2H])([2H])c1ccc2ccccc2c1</smiles>

(S)-Ethyl 3-(2-naphthyl)butanoate-2,3-d 2 dd.

The product was isolated by flash chromatography ( $\mathrm{Et}_{2} \mathrm{O} /$ hexanes 1:20) as colorless oil. $65 \mathrm{mg}, 90 \%$ yield. $[\alpha]^{23}=+22.4^{\circ}\left(\mathrm{c}=0.68, \mathrm{CHCl}_{3}\right)$

Ee: 91\%. Daicel Chiralcel OJ-H, n-hexane/isopropanol 95/5, flow rate $=0.5 \mathrm{~mL} / \mathrm{min}$.

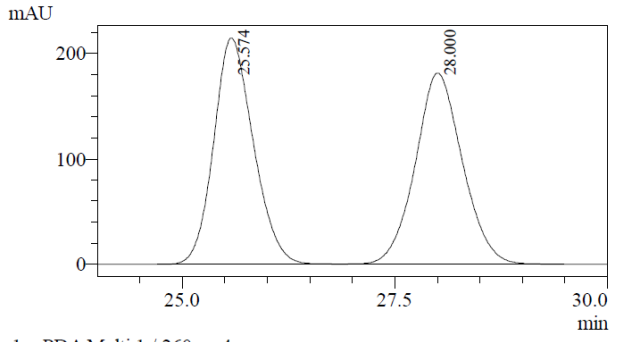

1 PDA Multi $1 / 260 \mathrm{~nm} 4 \mathrm{~nm}$

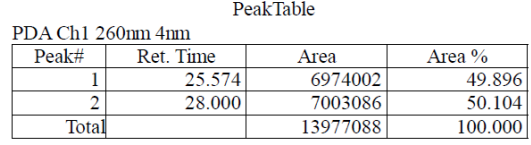

mAU

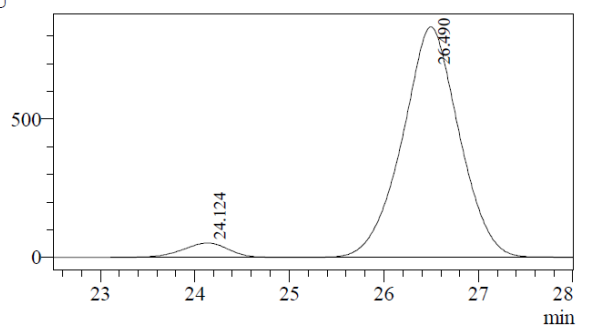

1 PDA Multi $1 / 200 \mathrm{~nm} 4 \mathrm{~nm}$

PeakTable

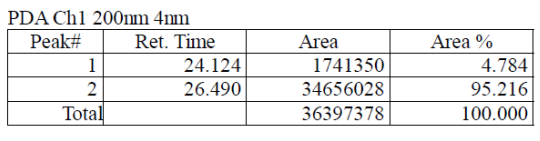

${ }^{1} \mathrm{H}$ NMR $\left(400 \mathrm{MHz}, \mathrm{CDCl}_{3}\right): \delta$ 7.86-7.75 (m, 3H), 7.72-7.64 (m, 1H), 7.52-7.36 (m, 3H), 4.19-4.00 (m, 2H), 3.54-3.38 (m, 0.08H), 2.72(s, 0.30H), 2.63(s, 0.71H), 1.14(s, 0.30), 1.28-1.11 (m, 3H). 
${ }^{2} \mathrm{H}$ NMR (61 MHz, $\left.\mathrm{CDCl}_{3}\right): \delta 3.48,2.75,2.66$.

${ }^{13} \mathrm{C}$ NMR (101 MHz, $\left.\mathrm{CDCl}_{3}\right): \delta$ 172.4, 143.2, 133.6, 132.4, 128.2, 127.7, 127.6, 126.0, 125.5, 125.4, 125.0, 60.3, $42.5(\mathrm{t}, J=19.8 \mathrm{~Hz}), 36.2(\mathrm{t}, J=20.0 \mathrm{~Hz}), 21.7(\mathrm{~d}, J=4.8 \mathrm{~Hz}), 14.2$.

LC-MS (ESI): Calcd for $\mathrm{C}_{16} \mathrm{H}_{17} \mathrm{D}_{2} \mathrm{O}_{2}{ }^{+}[\mathrm{M}+\mathrm{H}]^{+}$: 245.1. Found: 245.1 .<smiles>[2H]C(C)(C(=O)OCC)C([2H])([18OH])c1ccc(C)cc1</smiles>

\section{(S)-Ethyl 3-(4-tolyl)butanoate-2,3-d 2 2ed [1461-11-6].}

The product was isolated by flash chromatography ( $\mathrm{Et}_{2} \mathrm{O} /$ hexanes 1:20) as colorless oil. $40 \mathrm{mg}, 96 \%$ yield. $[\alpha]^{23}=+21.3^{\circ}\left(c=0.98, \mathrm{CDCl}_{3}\right)$.

Ee: 91\%. Daicel Chiralcel OJ-H, n-hexane/isopropanol 99/1, flow rate $=0.5 \mathrm{~mL} / \mathrm{min}$.

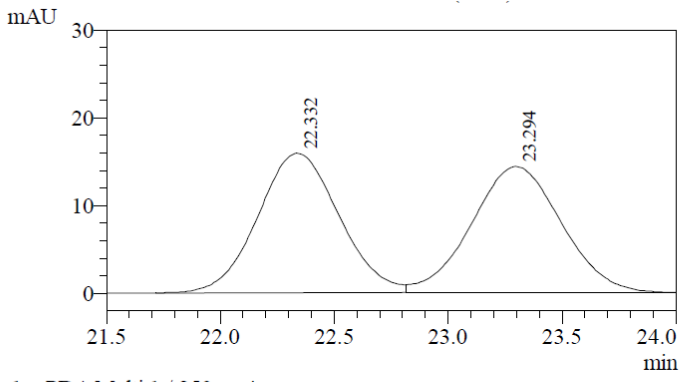

1 PDA Multi $1 / 250 \mathrm{~nm} 4 \mathrm{~nm}$

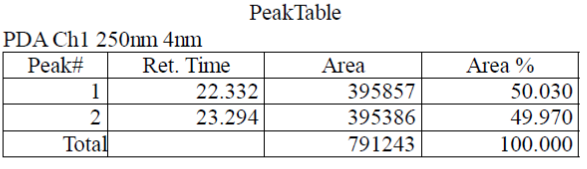

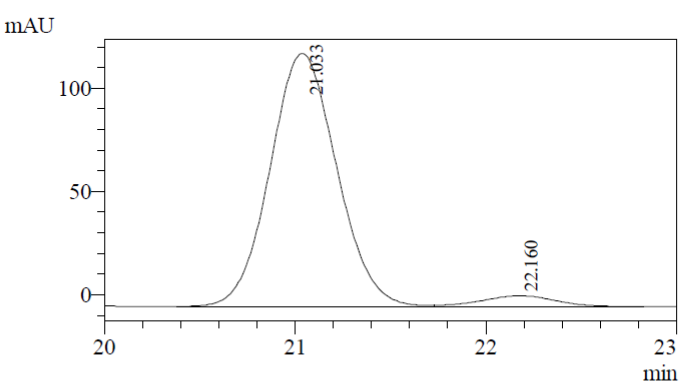

1 PDA Multi $1 / 205 \mathrm{~nm} 4 \mathrm{~nm}$

PeakTable

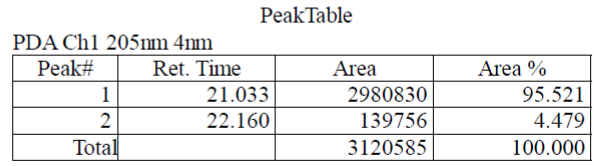

${ }^{1} \mathrm{H}$ NMR (400 MHz, $\left.\mathrm{CDCl}_{3}\right): \delta 7.12(\mathrm{~s}, 4 \mathrm{H}), 4.19-3.95$ (m, 2H), 3.31-3.17 (m, 0.03H), 2.57 (s, 0.29H), 2.51 (s, $0.72 \mathrm{H}), 2.33(\mathrm{~s}, 3 \mathrm{H}), 1.33-1.24(\mathrm{~m}, 3 \mathrm{H}), 1.24-1.15(\mathrm{~m}, 3 \mathrm{H})$.

${ }^{2} \mathrm{H}$ NMR (61 MHz, $\left.\mathrm{CDCl}_{3}\right): \delta 3.24,2.60,2.53$.

${ }^{13} \mathrm{C}$ NMR $\left(101 \mathrm{MHz}, \mathrm{CDCl}_{3}\right): \delta 172.5,142.7,135.8,129.1,126.6,60.2,42.7(\mathrm{t}, J=19.8 \mathrm{~Hz}), 35.6(\mathrm{t}, J=19.1$ Hz), $21.7(\mathrm{~d}, J=5.0 \mathrm{~Hz}), 21.0,14.18$.

LC-MS (ESI): Calcd for $\mathrm{C}_{13} \mathrm{H}_{17} \mathrm{D}_{2} \mathrm{O}_{2}^{+}[\mathrm{M}+\mathrm{H}]^{+}: 209.1$ Found: 209.0 .<smiles>[2H]C([2H])([18OH])C([2H])([2H])c1ccc(OC)cc1</smiles> 


\section{(S)-Ethyl 3-(4-anisyl)butanoate-2,3-d 2 2fd.}

The product was isolated by flash chromatography ( $\mathrm{Et}_{2} \mathrm{O} /$ hexanes $\left.1: 20\right)$ as colorless oil. $42 \mathrm{mg}, 95 \%$ yield. $[\alpha]^{23}=+21.2^{\circ}\left(c=1.4, \mathrm{CDCl}_{3}\right)$.

Ee: 90\%. Daicel Chiralcel OJ-H, $n$-hexane/isopropanol 99.5/0.5, flow rate $=0.5 \mathrm{~mL} / \mathrm{min}$.

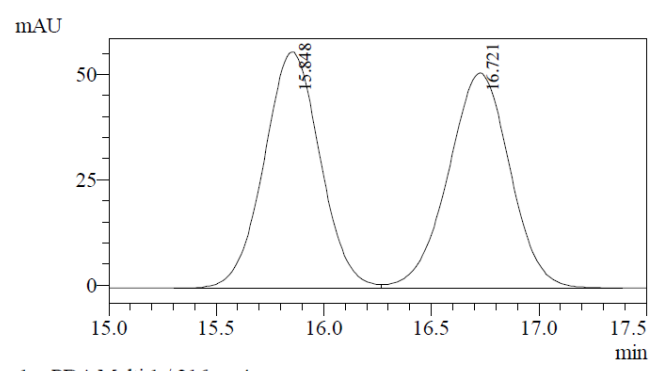

1 PDA Multi $1 / 216 \mathrm{~nm} 4 \mathrm{~nm}$

PeakTable

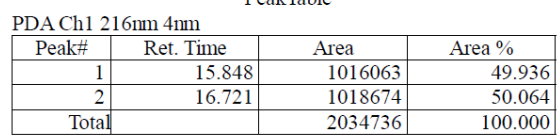

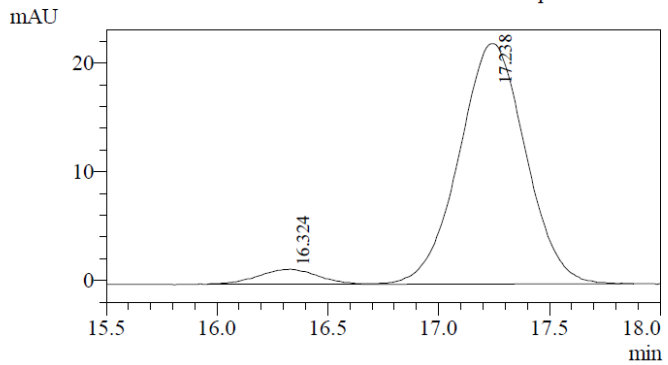

1 PDA Multi $1 / 254 \mathrm{~nm} 4 \mathrm{~nm}$

PeakTable

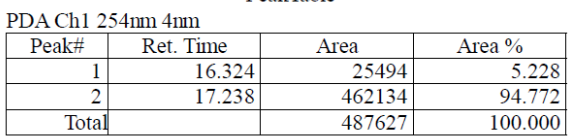

${ }^{1} \mathrm{H}$ NMR (400 MHz, $\left.\mathrm{CDCl}_{3}\right): \delta$ 7.19-7.08 (m, 2H), 6.94-6.78 (m, 2H), 4.16-4.00 (m, 2H), $3.78(\mathrm{~s}, 3 \mathrm{H}), 3.29-$

$3.17(\mathrm{~m}, 0.03 \mathrm{H}), 2.54(\mathrm{~s}, 0.30 \mathrm{H}), 2.49(\mathrm{~s}, 0.71 \mathrm{H}), 1.27(\mathrm{~s}, 3 \mathrm{H}), 1.23-1.12(\mathrm{~m}, 3 \mathrm{H})$.

${ }^{2} \mathrm{H}$ NMR (61 MHz, $\left.\mathrm{CDCl}_{3}\right): \delta 3.22,2.56,2.53$.

${ }^{13} \mathrm{C}$ NMR $\left(101 \mathrm{MHz}, \mathrm{CDCl}_{3}\right): \delta$ 172.5, 158.1, 137.8, 127.7, 113.8, 60.2, 55.2, 42.9 (t, $\left.J=19.9 \mathrm{~Hz}\right), 35.3(\mathrm{t}, J$

$=19.8 \mathrm{~Hz}), 21.9(\mathrm{~d}, J=4.8 \mathrm{~Hz}), 14.2$.

LC-MS (ESI): Calcd for $\mathrm{C}_{13} \mathrm{H}_{17} \mathrm{D}_{2} \mathrm{O}_{3}{ }^{+}[\mathrm{M}+\mathrm{H}]^{+}: 225.1$ Found: 225.1 .<smiles>[2H]C([2H])(C(=O)OCC)[C@@]([2H])([18OH])c1ccc2c(c1)OCO2</smiles>

\section{(S)-Ethyl 3-(3,4-methylendioxyphenyl)butanoate-2,3- $d_{2} 2 \mathrm{gd}$.}

The product was isolated by flash chromatography $\left(\mathrm{Et}_{2} \mathrm{O} /\right.$ hexanes 1:30) as colorless oil. $45 \mathrm{mg}, 96 \%$ yield. $[\alpha]^{23} \mathrm{D}=+26.5^{\circ}\left(c=1.2, \mathrm{CDCl}_{3}\right)$.

Ee: 90\%. Daicel Chiralcel OJ-H, $n$-hexane/isopropanol 90/10, flow rate $=1.0 \mathrm{~mL} / \mathrm{min}$. 

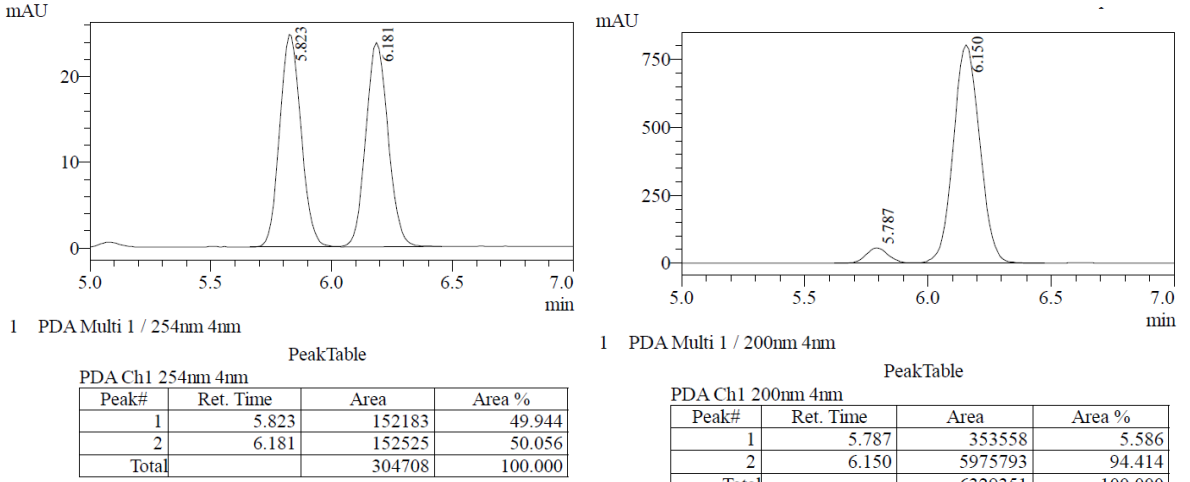

PDA Multi $1 / 200 \mathrm{~nm} 4 \mathrm{~nm}$

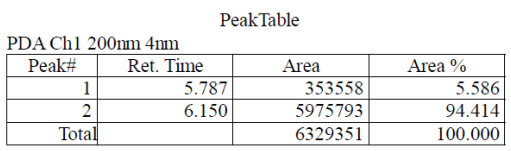

${ }^{1} \mathrm{H}$ NMR (400 MHz, $\left.\mathrm{CDCl}_{3}\right): \delta 6.75-6.61(\mathrm{~m}, 3 \mathrm{H}), 5.90(\mathrm{~s}, 2 \mathrm{H}), 4.07(\mathrm{q}, J=7.1 \mathrm{~Hz}, 2 \mathrm{H}), 3.28-3.08(\mathrm{~m}, 0.03 \mathrm{H})$, $2.51(\mathrm{~s}, 0.34 \mathrm{H}), 2.46(\mathrm{~s}, 0.69 \mathrm{H}), 1.24(\mathrm{~s}, 3 \mathrm{H}), 1.22-1.14(\mathrm{~m}, 3 \mathrm{H})$.

${ }^{2} \mathrm{H}$ NMR (61 MHz, $\left.\mathrm{CDCl}_{3}\right): \delta 3.17,2.51,2.47$.

${ }^{13} \mathrm{C}$ NMR $\left(101 \mathrm{MHz}, \mathrm{CDCl}_{3}\right): \delta 172.3,147.6,145.9,139.7,119.7,108.2,107.2,100.8,60.2,42.8(\mathrm{t}, J=19.8$ Hz), 35.9 (t, $J=19.9 \mathrm{~Hz}), 21.9(\mathrm{~d}, J=4.9 \mathrm{~Hz}), 14.19$.

LC-MS (ESI): Calcd for $\mathrm{C}_{13} \mathrm{H}_{15} \mathrm{D}_{2} \mathrm{O}_{4}{ }^{+}[\mathrm{M}+\mathrm{H}]^{+}: 239.1$ Found: 239.1 .<smiles>[2H]C([18OH])(C(=O)OCC)C([2H])([18OH])c1ccc(C(F)(F)F)cc1</smiles>

(S)-Ethyl 3-(4-trifluoromethylphenyl)butanoate-2,3- $d_{2} 2 \mathrm{hd}$.

The product was isolated by flash chromatography $\left(\mathrm{Et}_{2} \mathrm{O} /\right.$ hexanes $\left.1: 20\right)$ as colorless oil. $68 \mathrm{mg}, 87 \%$ yield. $[\alpha]^{23}{ }_{\mathrm{D}}=+23.7^{\circ}\left(c=0.24, \mathrm{CDCl}_{3}\right)$.

Ee: 93\%. Daicel Chiralcel IC-H, n-hexane/isopropanol 99.9/0.1, flow rate $=0.5 \mathrm{~mL} / \mathrm{min}$.

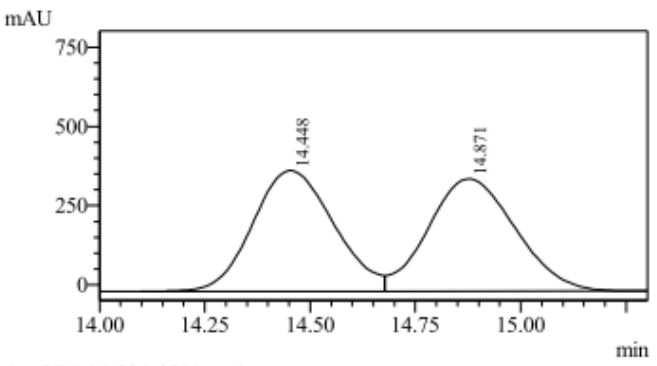

1 PDA Multi $1 / 200 \mathrm{~nm} 4 \mathrm{~nm}$

PeakTable

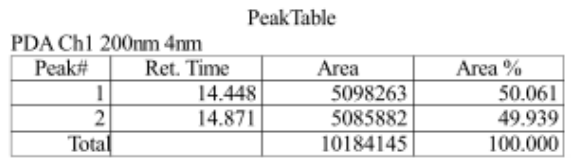

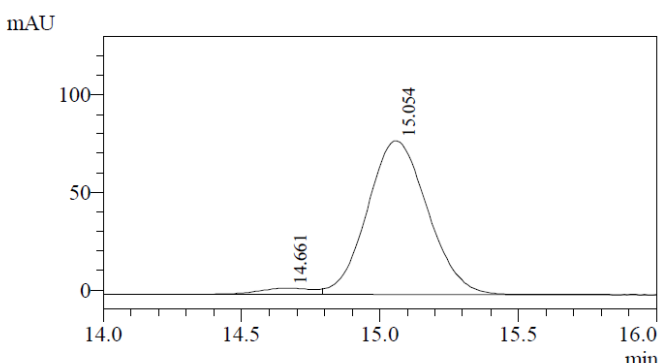

1 PDA Multi $1 / 210 \mathrm{~nm} 4 \mathrm{~nm}$

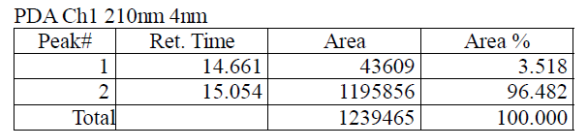

${ }^{1} \mathrm{H}$ NMR (400 MHz, $\left.\mathrm{CDCl}_{3}\right): \delta 7.55(\mathrm{~d}, J=8.1 \mathrm{~Hz}, 2 \mathrm{H}), 7.34(\mathrm{~d}, J=8.0 \mathrm{~Hz}, 2 \mathrm{H}), 4.12-4.02(\mathrm{~m}, 2 \mathrm{H}), 3.40-$ 
$3.28(\mathrm{~m}, 0.04 \mathrm{H}), 2.58(\mathrm{~s}, 0.33 \mathrm{H}), 2.55(\mathrm{~s}, 0.67 \mathrm{H}), 1.30(\mathrm{~d}, J=4.6 \mathrm{~Hz}, 3 \mathrm{H}), 1.20-1.03(\mathrm{~m}, 3 \mathrm{H})$.

${ }^{2} \mathrm{H}$ NMR (61 MHz, $\left.\mathrm{CDCl}_{3}\right): \delta 3.34,2.61,2.51$.

${ }^{3} \mathrm{C}$ NMR (101 MHz, $\left.\mathrm{CDCl}_{3}\right): \delta 171.9,149.7,128.7(\mathrm{q}, J=32.4 \mathrm{~Hz}), 127.2,125.4(\mathrm{q}, J=3.7 \mathrm{~Hz}), 124.2(\mathrm{q}, J=$ $271.8 \mathrm{~Hz}), 60.4,42.2$ (t, $J=19.7 \mathrm{~Hz}), 35.9$ (t, $J=20.1 \mathrm{~Hz}), 21.6$ (d, $J=4.5 \mathrm{~Hz}), 14.1$.

LC-MS (ESI): Calcd for $\mathrm{C}_{13} \mathrm{H}_{14} \mathrm{D}_{2} \mathrm{~F}_{3} \mathrm{O}_{2}[\mathrm{M}+\mathrm{H}]^{+}: 263.1$ Found: 263.1 .

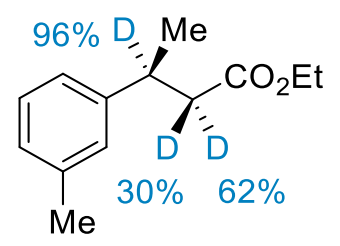

\section{(S)-ethyl 3-(3-tolyl)butanoate-2,3- $d_{2} 2$ id.}

The product was isolated by flash chromatography $\left(\mathrm{Et}_{2} \mathrm{O} /\right.$ hexanes 1:30) as colorless oil. $39 \mathrm{mg}, 95 \%$ yield. $[\alpha]^{23}=+24.0^{\circ}\left(c=1.4, \mathrm{CDCl}_{3}\right)$.

Ee: 92\%. Daicel Chiralcel OD-H, $n$-hexane/isopropanol 90/10, flow rate $=0.5 \mathrm{~mL} / \mathrm{min}$.

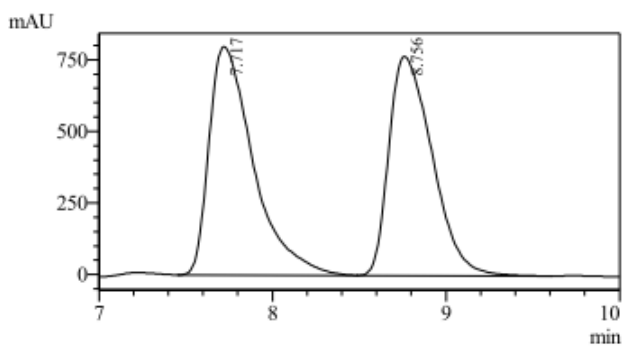

1 PDA Multi $1 / 200 \mathrm{~nm} 4 \mathrm{~nm}$
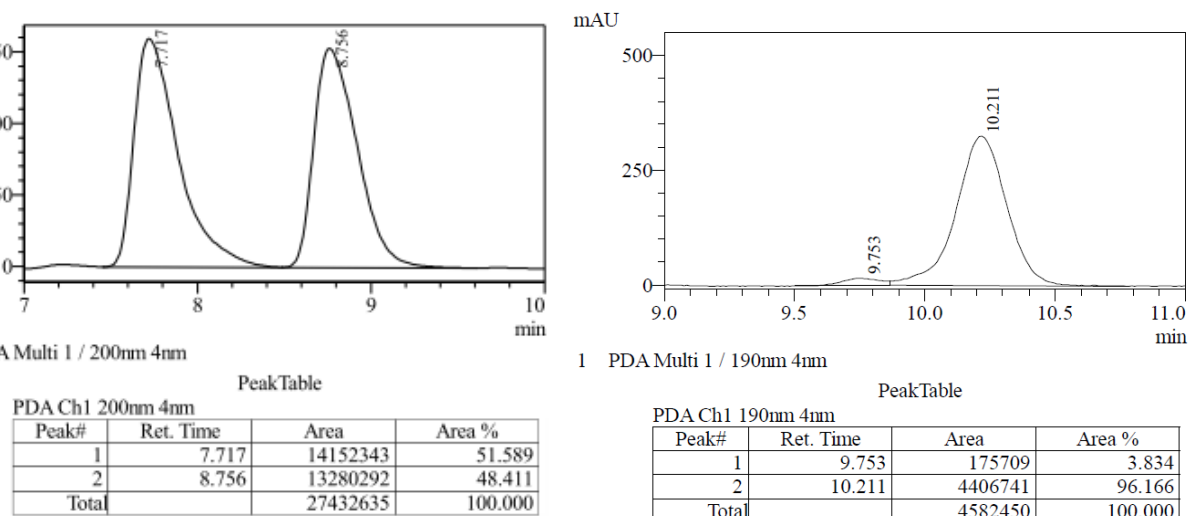

1 PDA Multi 1 / 190nm 4nm

PeakTable

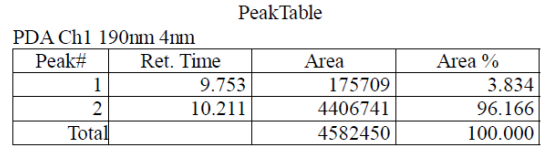

${ }^{1} \mathrm{H}$ NMR (400 MHz, CDCl $)$ : $\delta$ 7.25-7.13 (m, 1H), 7.08-6.94 (m, 3H), 4.16-4.04 (m, 2H), 3.36-3.13 (m, 0.04H), 2.59 (s, 1D), 2.52 (s, 1D), 2.35 (s, 3H), 1.30 (s, 3H), 1.24-1.07 (m, 3H).

${ }^{2} \mathrm{H}$ NMR (61 MHz, $\left.\mathrm{CDCl}_{3}\right): \delta 3.24,2.61,2.54$.

${ }^{13} \mathrm{C}$ NMR (101 MHz, $\left.\mathrm{CDCl}_{3}\right): \delta 172.5,145.7,138.0,128.4,127.6,127.1,123.7,60.2,42.6(\mathrm{t}, J=19.7 \mathrm{~Hz})$, $36.0(\mathrm{t}, J=19.9 \mathrm{~Hz}), 21.7(\mathrm{~d}, J=5.1 \mathrm{~Hz}), 14.2$.

LC-MS (ESI): Calcd for $\mathrm{C}_{13} \mathrm{H}_{17} \mathrm{D}_{2} \mathrm{O}_{2}[\mathrm{M}+\mathrm{H}]^{+}$: 209.2 Found: 209.1 .

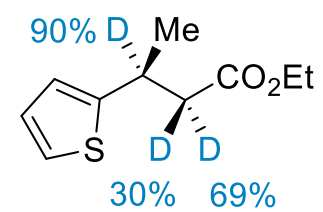




\section{(S)-Ethyl 3-(2-thienyl)butanoate-2,3- $d_{2} 2 \mathrm{jd}$.}

The product was isolated by flash chromatography ( $\mathrm{Et}_{2} \mathrm{O} /$ hexanes $\left.1: 20\right)$ as colorless oil. $37 \mathrm{mg}, 93 \%$ yield. $[\alpha]^{23}=+17.2^{\circ}\left(c=1.44, \mathrm{CDCl}_{3}\right)$.

Ee: $89 \%$ ee. Daicel Chiralcel OD-H, $n$-hexane/isopropanol 99.5/0.5, flow rate $=0.5 \mathrm{~mL} / \mathrm{min}$.

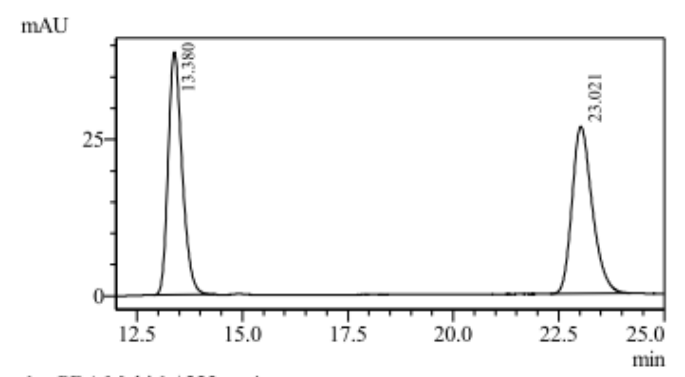

1 PDA Multi $1 / 223 \mathrm{~nm} 4 \mathrm{~nm}$

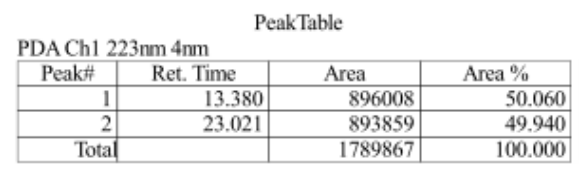

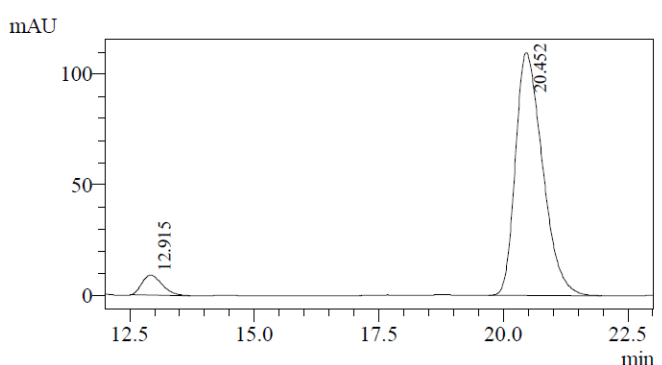

1 PDA Multi $1 / 225 \mathrm{~nm} 4 \mathrm{~nm}$

PeakTable

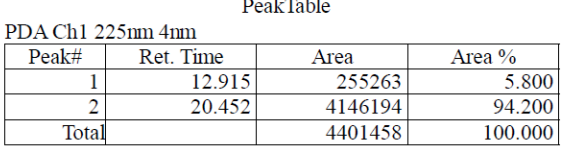

${ }^{1} \mathrm{H}$ NMR (400 MHz, $\left.\mathrm{CDCl}_{3}\right): \delta 7.13(\mathrm{dd}, J=5.1,1.1 \mathrm{~Hz}, 1 \mathrm{H}), 6.91(\mathrm{dd}, J=5.1,3.5 \mathrm{~Hz}, 1 \mathrm{H}), 6.84(\mathrm{dd}, J=3.5$, $1.1 \mathrm{~Hz}, 1 \mathrm{H}), 4.12(\mathrm{q}, J=7.1 \mathrm{~Hz}, 2 \mathrm{H}), 3.63-3.55(\mathrm{~m}, 0.10 \mathrm{H}), 2.66(\mathrm{~s}, 0.31 \mathrm{H}), 2.54(\mathrm{~s}, 0.70 \mathrm{H}), 1.38(\mathrm{~s}, 3 \mathrm{H}), 1.22$ $(\mathrm{t}, J=7.1 \mathrm{~Hz}, 3 \mathrm{H})$.

${ }^{2} \mathrm{H}$ NMR (61 MHz, $\left.\mathrm{CDCl}_{3}\right): \delta 3.59,2.68,2.56$.

${ }^{13} \mathrm{C}$ NMR (101 MHz, $\left.\mathrm{CDCl}_{3}\right): \delta$ 172.0, 149.6, 126.6, 123.0, 122.9, 60.42, $43.5(\mathrm{t}, J=19.8 \mathrm{~Hz}), 31.6(\mathrm{t}, J=20.1$ $\mathrm{Hz}), 22.52(\mathrm{~d}, J=4.7 \mathrm{~Hz}), 14.20$.

LC-MS (ESI): Calcd for $\mathrm{C}_{10} \mathrm{H}_{13} \mathrm{D}_{2} \mathrm{O}_{2} \mathrm{~S}[\mathrm{M}+\mathrm{H}]^{+}$: 201.1 Found: 201.1.<smiles>[2H]C([2H])(C(=O)OCC)C([2H])([2H])c1cccnc1</smiles>

(S)-Ethyl 3-(3-pyridyl)butanoate-2,3-d $2 \mathrm{kd}$.

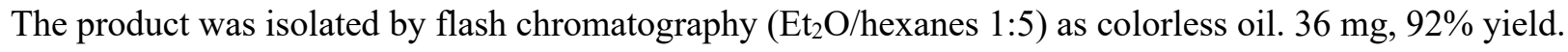
$[\alpha]^{23} \mathrm{D}=+27.0^{\circ}\left(c=1.0, \mathrm{CDCl}_{3}\right)$.

Ee: 92\%. Daicel Chiralcel AS-H, $n$-hexane/isopropanol 98/2, flow rate $=0.5 \mathrm{~mL} / \mathrm{min}$. 

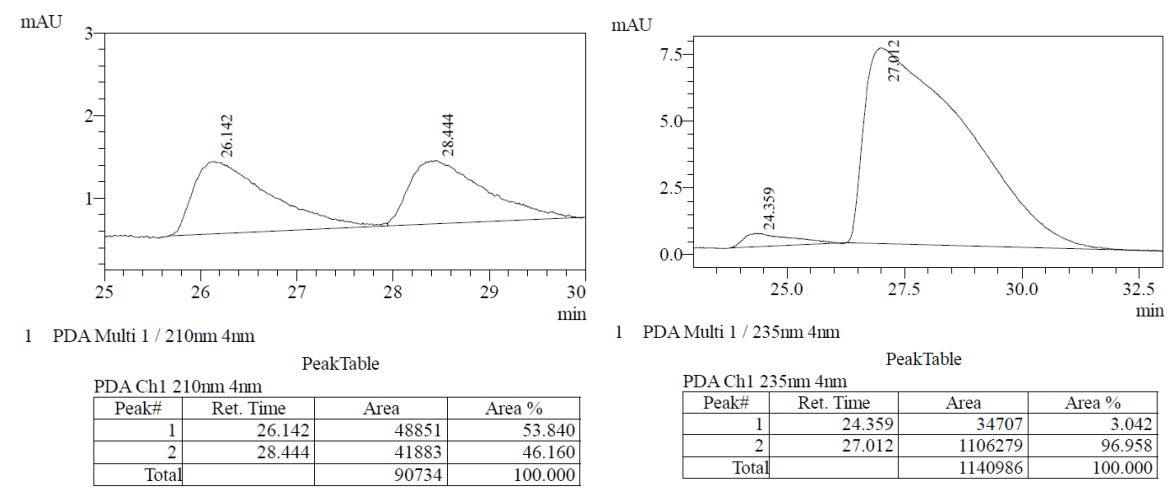

1 PDA Multi $1 / 235 \mathrm{~nm} 4 \mathrm{~nm}$

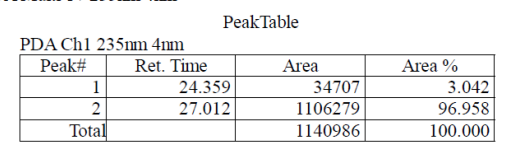

${ }^{1} \mathrm{H}$ NMR (400 MHz, $\left.\mathrm{CDCl}_{3}\right): \delta 8.53-8.36(\mathrm{~m}, 2 \mathrm{H}), 7.50(\mathrm{~d}, J=7.8 \mathrm{~Hz}, 1 \mathrm{H}), 7.23-7.10(\mathrm{~m}, 1 \mathrm{H}), 4.03(\mathrm{q}, J=$

$7.1 \mathrm{~Hz}, 2 \mathrm{H}), 3.33-3.15(\mathrm{~m}, 0.04 \mathrm{H}), 2.56(\mathrm{~s}, 1 \mathrm{H}), 1.28(\mathrm{~s}, 3 \mathrm{H}), 1.13(\mathrm{t}, J=7.1 \mathrm{~Hz}, 3 \mathrm{H})$.

${ }^{2} \mathrm{H}$ NMR (61 MHz, $\left.\mathrm{CDCl}_{3}\right): \delta 3.25,2.55$.

${ }^{13} \mathrm{C}$ NMR $\left(101 \mathrm{MHz}, \mathrm{CDCl}_{3}\right): \delta 171.8,148.8,147.9,140.7,134.1,123.4,60.4,42.1(\mathrm{t}, J=19.8 \mathrm{~Hz}), 33.6(\mathrm{t}, J$ $=19.9 \mathrm{~Hz}), 21.46(\mathrm{~d}, J=4.0 \mathrm{~Hz}), 14.11$.

LC-MS (ESI): Calcd for $\mathrm{C}_{11} \mathrm{H}_{14} \mathrm{D}_{2} \mathrm{NO}_{2}[\mathrm{M}+\mathrm{H}]^{+}: 196.1$ Found: 196.1.<smiles>[2H][C@]([18OH])(C(=O)OCC)[13CH]1[13CH]CCc2ccccc21</smiles>

\section{(S)-Ethyl 2-(1-tetralinyl)acetate-2,3- $d_{2 .}$ 2ld.}

The $(E)$-isomer of starting material was used. The product was isolated by flash chromatography $\left(\mathrm{Et}_{2} \mathrm{O} / \mathrm{hexanes}\right.$ 1:15) as colorless oil. $40 \mathrm{mg}, 93 \%$ yield.

$[\alpha]^{23} \mathrm{D}=+1.3^{\circ}\left(c=1.2, \mathrm{CDCl}_{3}\right)$.

Ee: $84 \%$. Daicel Chiralcel OJ-H, $n$-hexane/isopropanol 98/2, flow rate $=1.0 \mathrm{~mL} / \mathrm{min}$.
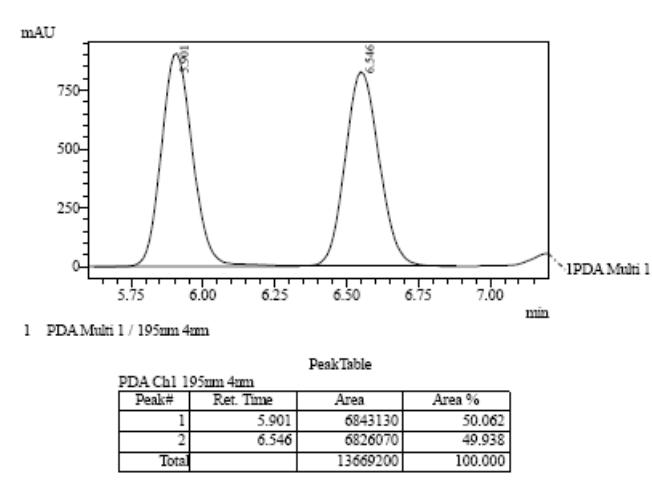

$\mathrm{mAU}$

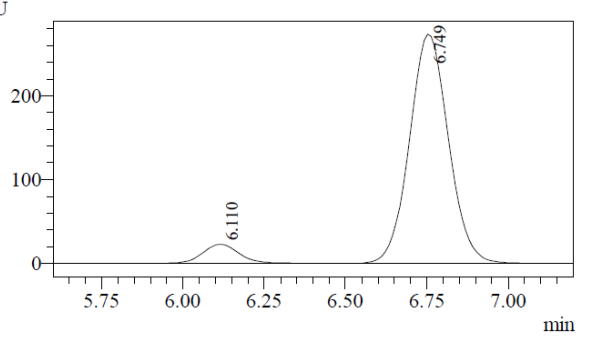

PDA Multi $1 / 200 \mathrm{~nm} 4 \mathrm{~nm}$

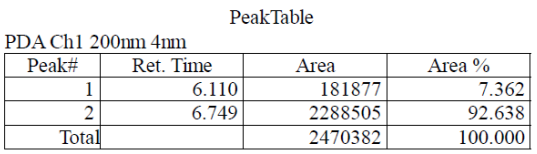

${ }^{1} \mathrm{H}$ NMR (400 MHz, $\left.\mathrm{CDCl}_{3}\right): \delta$ 7.23-7.04 (m, 4H), 4.19 (q, $\left.J=7.1 \mathrm{~Hz}, 2 \mathrm{H}\right), 3.43-3.30(\mathrm{~m}, 0.02 \mathrm{H}), 2.88-2.71$ (m, 2H), $2.69(\mathrm{~s}, 0.39 \mathrm{H}), 2.52(\mathrm{~s}, 0.61 \mathrm{H}), 1.98-1.67(\mathrm{~m}, 4 \mathrm{H}), 1.29(\mathrm{t}, J=7.1 \mathrm{~Hz}, 3 \mathrm{H}), 0.98-0.71(\mathrm{~m}, 1 \mathrm{H})$ 
${ }^{2} \mathrm{H}$ NMR (61 MHz, $\left.\mathrm{CDCl}_{3}\right): \delta 3.36,2.71,2.54$.

${ }^{13} \mathrm{C}$ NMR (101 MHz, $\mathrm{CDCl}_{3}$ ): $\delta 172.9$ (s), 139.3 (s), 137.2 (s), 129.3 (s), 128.3 (s), 126.0 (s), 125.8 (s), 60.4 (s), $41.7(\mathrm{t}, J=19.8 \mathrm{~Hz}), 34.1(\mathrm{t}, J=20.0 \mathrm{~Hz}), 29.53(\mathrm{~s}), 27.98$ (d, $J=4.7 \mathrm{~Hz}), 19.52(\mathrm{~s}), 14.29$ (s).

LC-MS (ESI): Calcd for $\mathrm{C}_{14} \mathrm{H}_{17} \mathrm{D}_{2} \mathrm{O}_{2}[\mathrm{M}+\mathrm{H}]^{+}: 221.2$ Found: 221.2.<smiles>[2H][C@]([18OH])(C(=O)OCC)[C@@H]1CCOc2ccccc21</smiles>

(S)-Ethyl 2-(4-chromanyl)acetate-2,3- $d_{2 .} 2 \mathrm{md}$.

The $(E)$-isomer of starting material was used. The product was isolated by flash chromatography (EtOAc/hexanes 1:4) as colorless oil. $41 \mathrm{mg}$, 94\% yield.

$[\alpha]^{23}=+0.46^{\circ}\left(c=2.35, \mathrm{CDCl}_{3}\right)$.

Ee: 82\%. Daicel Chiralcel OJ-H, $n$-hexane/isopropanol 90/10, flow rate $=1.0 \mathrm{~mL} / \mathrm{min}$.
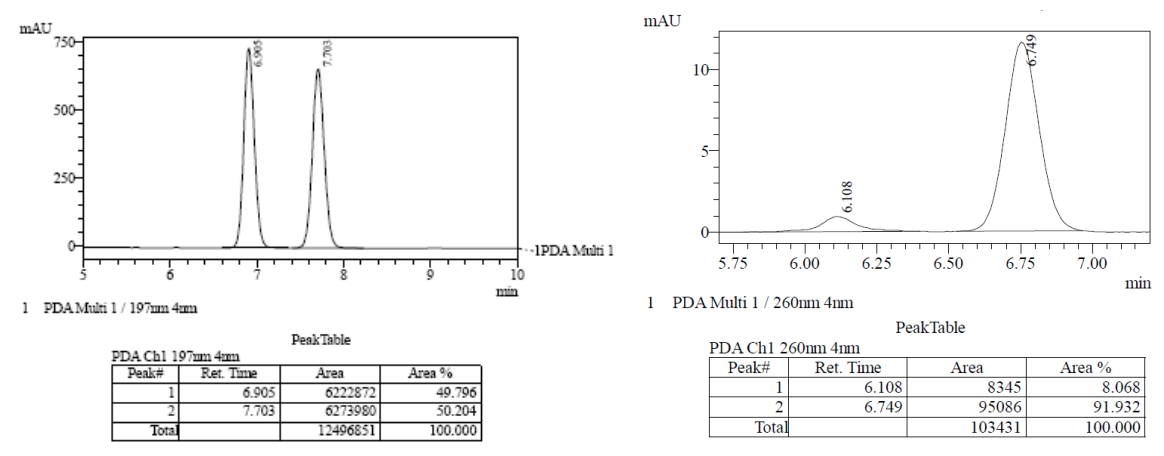

${ }^{1} \mathrm{H}$ NMR (400 MHz, $\mathrm{CDCl}_{3}$ ): $\delta$ 7.19-7.03 (m, 2H), 6.91-6.76 (m, 2H), 4.24-4.12 (m, 4H), 3.41-3.31 (m, 0.02H), $2.77(\mathrm{~s}, 0.32 \mathrm{H}), 2.50(\mathrm{~s}, 0.69 \mathrm{H}), 2.23-2.08(\mathrm{~m}, 1 \mathrm{H}), 1.98-1.74(\mathrm{~m}, 1 \mathrm{H}), 1.28(\mathrm{t}, J=7.1 \mathrm{~Hz}, 3 \mathrm{H})$.

${ }^{2} \mathrm{H}$ NMR $\left(61 \mathrm{MHz}, \mathrm{CDCl}_{3}\right): \delta 3.35,2.79,2.53$.

${ }^{13} \mathrm{C}$ NMR $\left(101 \mathrm{MHz}, \mathrm{CDCl}_{3}\right): \delta 172.2,154.6,128.7,127.9,124.5,120.4,117.1,63.2,60.6,41.0(\mathrm{t}, J=19.7$ $\mathrm{Hz}$ ), 30.0 (t, $J=18.2 \mathrm{~Hz}), 27.2(\mathrm{~d}, J=3.6 \mathrm{~Hz}), 14.3$.

LC-MS (ESI): Calcd for $\mathrm{C}_{13} \mathrm{H}_{15} \mathrm{D}_{2} \mathrm{O}_{3}[\mathrm{M}+\mathrm{H}]^{+}: 223.1$ Found: 223.1 .<smiles>CCOC(=O)C([18OH])([18OH])C([18OH])([18OH])c1ccccc1</smiles>

(S)-Ethyl 3-phenylpentanoate-2,3- $d_{2}$ 2nd [2845-23-0]. 
The product was isolated by flash chromatography ( $\mathrm{Et}_{2} \mathrm{O} /$ hexanes $\left.1: 20\right)$ as colorless oil. $38 \mathrm{mg}, 93 \%$ yield. $[\alpha]^{23}{ }_{\mathrm{D}}=+14.3^{\circ}\left(c=1.2, \mathrm{CDCl}_{3}\right)$.

Ee: $96 \%$. Daicel Chiralcel OD-H, $n$-hexane/isopropanol 99/1, flow rate $=0.5 \mathrm{~mL} / \mathrm{min}$.

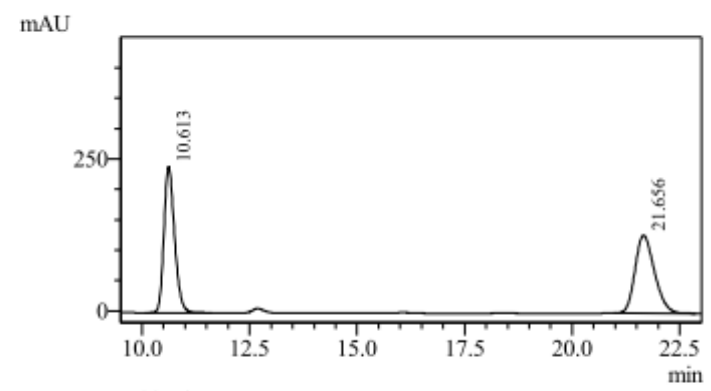

1 PDA Multi $1 / 200 \mathrm{~nm} 4 \mathrm{~nm}$
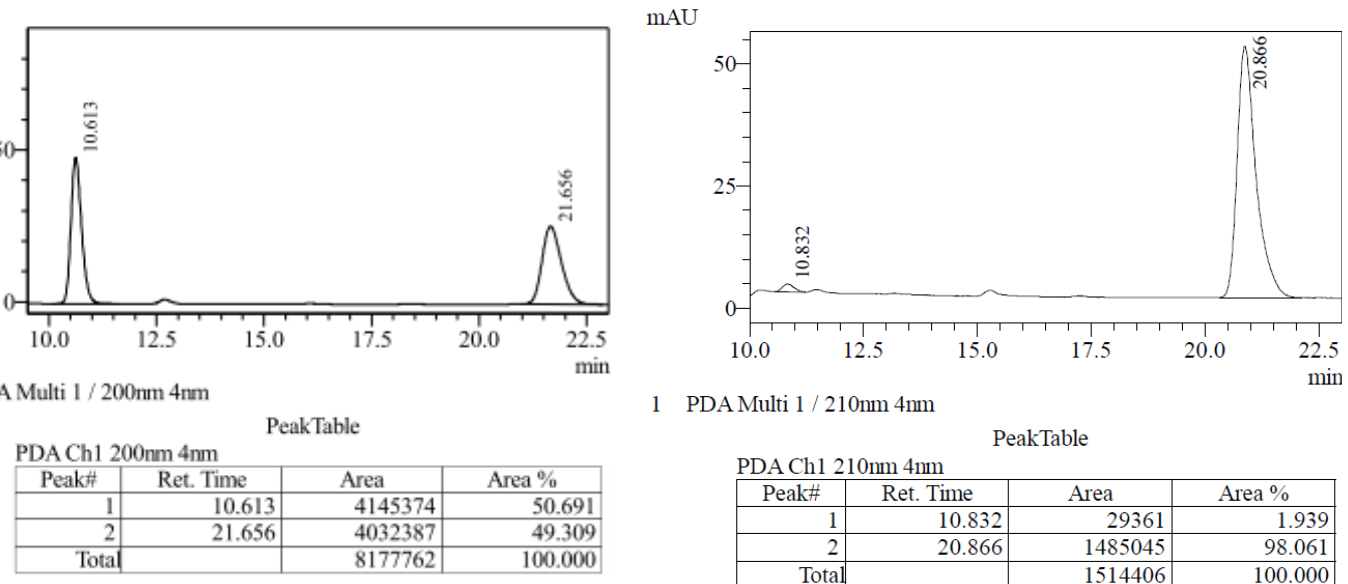

1 PDA Multi $1 / 210 \mathrm{~nm} 4 \mathrm{~nm}$

PDA Ch1 $210 \mathrm{~nm}$ 4nm
\begin{tabular}{|r|r|r|r|}
\hline Peak\# & Ret. Time & Area & \multicolumn{1}{|c|}{ Area $\%$} \\
\hline 1 & 10.832 & 29361 & 1.939 \\
\hline 2 & 20.866 & 1485045 & 98.061 \\
\hline Total & & 1514406 & 100.000 \\
\hline
\end{tabular}

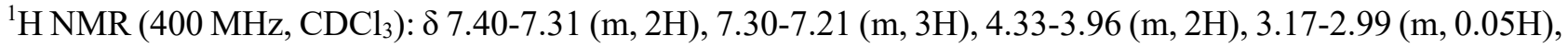
2.68 (s, 0.74), 2.61 (s, 0.26), 1.91-1.57 (m, 2H), 1.20 (td, $J=7.1,1.9 \mathrm{~Hz}, 3 \mathrm{H}), 0.87$ (t, $J=7.4 \mathrm{~Hz}, 3 \mathrm{H})$.

${ }^{2} \mathrm{H} \mathrm{NMR}\left(61 \mathrm{MHz}, \mathrm{CDCl}_{3}\right): \delta 3.00,2.64,2.57$.

${ }^{13} \mathrm{C}$ NMR $\left(101 \mathrm{MHz}, \mathrm{CDCl}_{3}\right): \delta 172.5,143.9,128.3,127.5,126.4,60.17,43.5(\mathrm{t}, J=19.9 \mathrm{~Hz}), 41.1(\mathrm{t}, J=19.9$

Hz), $29.0(\mathrm{~d}, J=3.9 \mathrm{~Hz}), 14.1,11.9$.

LC-MS (ESI): Calcd for $\mathrm{C}_{13} \mathrm{H}_{17} \mathrm{D}_{2} \mathrm{O}_{2}{ }^{+}[\mathrm{M}+\mathrm{H}]^{+}$: 209.1 Found: 209.1 .

\section{Reference}

(1) Guo, S.; Yang, P.; Zhou, J. S. Chem. Commun. 2015, 51, 12115.

(2) Yang, P.; Xu, H.; Zhou, J. S. Angew. Chem., Int. Ed. 2014, 53, 12210.

(3) Giovanni, F.; Claudio, F.; Stefano, S. Eur. J. Org. Chem. 2009, 2009, 6160.

(4) Sandra, V.; Nicole, S.; Stefan, T.; Sabine, L. Eur. J. Org. Chem. 2013, 2013, 1580.

(5) Huang, J.; Hong, M.; Wang, C. C.; Kramer, S.; Lin, G. Q.; Sun, X. W. J. Org. Chem. 2018, 83, 12838.

(6) Ouyang, G. H.; He, Y. M.; Li, Y.; Xiang, J. F.; Fan, Q. H. Angew. Chem., Int. Ed. 2015, 54, 4334. 